\title{
Base models for color halftone reproduction
}

\author{
Roger D. Hersch \\ Ecole Polytechnique Fédérale de Lausanne (EPFL), \\ School of Computer and Communication Sciences, Lausanne, Switzerland
}

\author{
Mathieu Hébert \\ Université de Lyon, Université Jean Monnet de Saint-Etienne, CNRS UMR 5516 \\ Laboratoire Hubert Curien, Saint-Etienne, France.
}

\begin{abstract}
One of the main challenges for the characterization of printing systems consists, for a given substrate, in establishing the relationship between surface coverages of the selected set of inks and the spectral reflectances of the printed ink halftones. Knowledge of the spectral reflectances of print halftones enables deducing their colors. Models enabling the prediction of spectral reflectances as a function of surface coverages of the inks are helpful in characterizing printers and in creating printer profiles. The presented base models for color halftone reproduction are either surface models such as the Yule-Nielsen modified spectral Neugebauer model or physically inspired models accounting for the interaction of light, inks and substrate such as the Clapper-Yule model. As a complement to reflectance prediction models, we introduce ink spreading models that account for the interaction between superposed ink halftones and the substrate. They help understanding and modeling the dot gain phenomena present in most printing systems. Finally, we compare the prediction accuracies of the different models for different printing technologies, paper types and screen frequencies.
\end{abstract}

\section{Key words}

Interaction of light, ink halftones and substrate, printer characterization, color prediction model, spectral reflectance prediction, Demichel equations, Yule-Nielsen modified spectral Neugebauer model (YNSN), cellular Yule-Nielsen model, two-by-two dot centering model, Clapper-Yule model, low-scattering Clapper-Yule model, Williams-Clapper model, ink spreading models, color prediction model accuracy. 


\section{INTRODUCTION}

Today's color reproduction systems aim at producing without manual intervention color images, which are as close as possible to the original images. Color images shall be transferable from one device to the next and still look similar when shown on a display or printed on paper. In order to be able to create on a given printer images whose colors are as faithfully reproduced as possible, it is necessary to characterize that printer, i.e. to specify for a given selection of paper, inks, and halftones, the color response it provides when asked to print with given amounts of inks. Once characterized, a printer can be inserted into a color reproduction workflow.

There are many additional reasons for characterizing a printer. Printer manufacturers may for example want to dynamically characterize their printers in order to verify that the printer operation mode has not drifted away. Depending on the result of this characterization, they may also adapt printing parameters in order to ensure that for given control values the printed colors remain identical. Finally, thanks to color prediction models, the usage of the inks can be optimized. In modern 6,9 or 12 ink printers, there are many possibilities of printing a given color. The color prediction model may help the software to decide, according to given optimization criteria, which set of inks to select in order to print a given color within a given context. Possible optimization criteria can be the minimization ink usage [1], the low visibility of halftones [2], or the minimization of metamerism when observing a print under various light sources [3].

Since more than 50 years, there have been attempts to create models predicting the color of printed halftone images. In order to offer accurate predictions, such models need to take into account both the interactions between the inks and the paper and between the light and the halftone print. Many different phenomena influence the reflection spectrum of a color halftone patch printed on a diffusely reflecting substrate (e.g. paper). These phenomena comprise the surface (Fresnel) reflection at the interface between the air and the paper, light scattering and reflection within the substrate (i.e. the paper bulk), the internal (Fresnel) reflections at the interface between the paper and the air and possibly the fluorescent emission of the optically brightened paper substrate. The lateral scattering of light within the paper substrate and the internal reflections at the interface between the paper and the air are responsible for what is generally called the optical dot gain, known as the Yule-Nielsen effect. In addition, due to the 
printing process, the deposited ink surface coverage is generally larger than the nominal coverage that the printer is asked to print, yielding a "physical" dot gain (also called "mechanical" dot gain). The ink surface coverages effectively printed depend on the nature of the printing process, on the inks, on the paper surface, on the paper bulk, and also on the specific superpositions of individual ink halftones.

We present a number of reflectance prediction models. Their ability to achieve a given prediction accuracy depends on the context, i.e. the combination of print technology, substrate, inks and halftoning methods. We restrict the presentation to mainstream models relying on classical halftoning techniques, where the halftone layers are printed independently one from another, i.e. classical mutually rotated clustered dot screens, blue noise halftones and errordiffusion halftones.

\section{CATEGORIES OF REFLECTANCE PREDICTION MODELS}

In order to guide the reader, let us give first a brief overview of the categories of color print prediction models and then present basic assumptions about ink halftones and colorants on which these color prediction models rely. In the present context, colorants, also called Neugebauer primaries, are defined as being formed by the individual solid inks, by solid ink superpositions and by the paper white. Color halftones are formed by the juxtaposed colorants formed by the superposition of the individual ink dot halftone layers (see Figure 16).

Surface models rely on the surfaces of the colorants formed as superpositions of ink halftones. Such surface models assume that the reflected (or transmitted) light is a function of the effective surface coverage of the colorants or of base patterns forming an ink halftone. These surface models don't rely on the detailed analysis of the light propagation and light attenuation within the print. They generally consist of an empirical formula creating the relationship between colorant surface coverages and reflected light.

Physically inspired halftone print reflection models rely on a more detailed analysis of the interaction between the light and the print. The light paths within a halftone print are analyzed and the resulting attenuation of light is described by mathematical equations. 
Ink spreading models aim at characterizing the effective surface of a colorant or of an ink dot, after it has been printed at a given desired nominal surface coverage (control value). The dot gain is defined as the difference between the effective surface coverage comprising that dot gain and the nominal surface coverage. In case that the effective surface coverage is obtained with a prediction model that accounts for the optical dot gain, it characterizes how much an ink dot spreads out when printed on paper or when printed on another ink.

Further reflection models described in the chapters that follow concern point spread based models, light propagation probability models, Monte-Carlo based light transportation models as well as Kubelka-Munk inspired two-flux models.

\section{Independence of ink layers and calculation of surface coverages}

For the spectral prediction models described below, we calculate surface coverages of the colorants formed by inks and superpositions of inks by making the assumption that the ink layers, for example the cyan, magenta, yellow and black layers, are spatially laid out independently one from another. As illustration, consider a first layer (cyan) whose halftone dot grows horizontally and a second ink layer (magenta) whose halftone dot grows vertically.

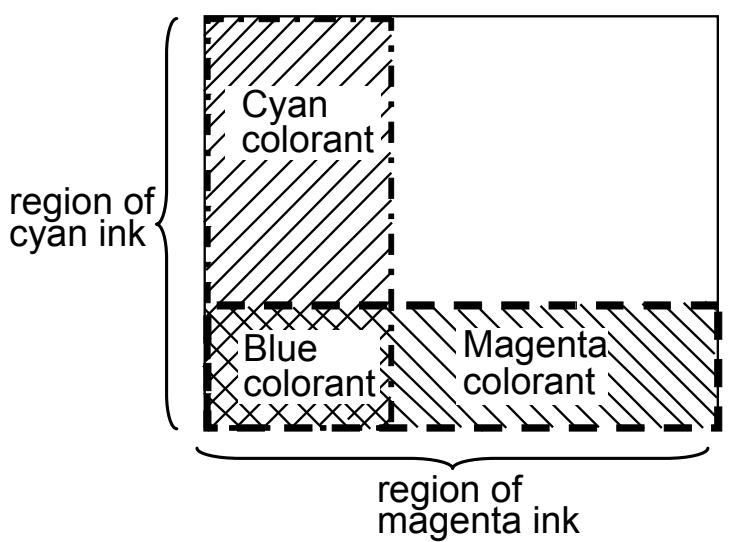

cyan ink surface coverage : $\quad c$ magenta ink surface coverage: $m$ cyan colorant surface coverage: $a_{c}$ magenta colorant surface coverage: $a_{m}$ blue colorant surface coverage: $a_{b}$ white colorant surface coverage: $a_{w}$

Figure 1: Example of a halftone with two ink halftone dots, forming four different colorant surfaces.

Let us assume that the total surface of the halftone element of Figure 1 is one. The respective surface coverages of the cyan and magenta inks are respectively $c$ and $m$, having values between 0 and 1. Let us assume that light rays are thrown into the halftone element surface, and that their probability to fall within a specific location of that surface follows a uniform 
distribution. Then the probability $P(c)$ to fall on a cyan ink dot of surface coverage $c$ is equal to $c$ and the probability $P(m)$ to fall on a magenta ink dot of surface coverage $m$ is equal to $m$.

The visible colorants form a "puzzle" of juxtaposed colors, composed of the paper, the inks and their superpositions. In case of Figure 1, the colorant cyan is the region covered by ink cyan only, without superposed magenta and the colorant magenta is the region covered by ink magenta only, without superposed cyan. Colorant white is the region covered by no ink and colorant blue is the region covered by both cyan and magenta inks.

If the ink halftone dots are laid out independently, as in Figure 1, the probability of a light ray to hit the cyan colorant is the probability of hitting the cyan ink multiplied by the probability of not hitting the magenta ink. Similar considerations apply for the other colorants. We obtain

$$
\begin{aligned}
& P(\text { Colorant }=\text { cyan })=P(c) \cdot(1-P(m)) \\
& P(\text { Colorant }=\text { magenta })=P(m) \cdot(1-P(c)) \\
& P(\text { Colorant }=\text { blue })=P(c) \cdot P(m) \\
& P(\text { Colorant }=\text { white })=(1-P(c)) \cdot(1-P(m))
\end{aligned}
$$

Since for an incoming photon the probability to hit a given colorant is proportional to the surface coverage of that colorant, we can deduce the surface coverages $a_{c}, a_{m}, a_{b}, a_{w}$ of the individual colorants cyan, magenta, blue and white:

$$
\begin{aligned}
& a_{c}=P(\text { Colorant = cyan })=P(c) \cdot(1-P(m))=c \cdot(1-m) \\
& a_{m}=P(\text { Colorant }=\text { magenta })=P(m) \cdot(1-P(c))=(1-c) \cdot m \\
& a_{b}=P(\text { Colorant }=\text { blue })=P(c) \cdot P(m)=c \cdot m \\
& a_{w}=P(\text { Colorant }=\text { white })=(1-P(c)) \cdot(1-P(m))=(1-c) \cdot(1-m)
\end{aligned}
$$

This simple calculation of colorant surfaces has been deduced by Demichel [4].

Along the same reasoning line, with 3 inks, we obtain equations (3) expressing the 8 colorant surface coverages as a function of ink surface coverages of the cyan $(c)$, magenta $(m)$ and yellow $(y)$ ink surface coverages. The colorants are white, cyan, magenta, yellow, red (superposed magenta and yellow), green (superposed cyan and yellow), blue (superposed magenta and cyan), and black (superposed cyan, magenta and yellow).

$\begin{array}{llll}\text { white: } & a_{w}=(1-c)(1-m)(1-y) ; & \text { cyan: } & a_{c}=c(1-m)(1-y) \\ \text { magenta: } & a_{m}=(1-c) m(1-y) ; & \text { yellow: } & a_{y}=(1-c)(1-m) y \\ \text { red: } & a_{r}=(1-c) m y ; & \text { green: } & a_{g}=c(1-m) y \\ \text { blue: } & a_{b}=c m(1-y) ; & \text { black: } & a_{k}=c m y\end{array}$


With 4 inks we obtain similar expressions for the 16 colorants, where $k$ is the surface coverage of the black ink :

white: $\quad a_{w}=(1-c)(1-m)(1-y)(1-k)$; cyan: $a_{c}=c(1-m)(1-y)(1-k)$

magenta: $\quad a_{m}=(1-c) m(1-y)(1-k) ; \quad$ yellow: $a_{y}=(1-c)(1-m) y(1-k)$

red: $\quad a_{m y}=(1-c) m y(1-k) ; \quad$ green: $\quad a_{c y}=c(1-m) y(1-k)$

blue: $\quad a_{c m}=c m(1-y)(1-k) ; \quad$ chromatic black: $a_{c m y}=c m y(1-k)$

black: $\quad a_{w}=(1-c)(1-m)(1-y) k ; \quad$ cyanBlack: $a_{c k}=c(1-m)(1-y) k$

magentaBlack: $a_{m k}=(1-c) m(1-y) k ; \quad$ yellowBlack: $a_{y k}=(1-c)(1-m) y k$

redBlack: $\quad a_{m y k}=(1-c) m y k ; \quad$ greenBlack: $a_{c y k}=c(1-m) y k$

blueBlack: $\quad a_{c m k}=c m(1-y) k ; \quad$ totalBlack: $a_{c m y k}=c m y k$

These equations are valid in all cases where the inks halftone dots are independently laid out, e.g. for stochastic screening, for error diffusion, as well as for mutually rotated clustered dot screens [5].

\section{SURFACE COLOR PREDICTION MODELS}

Surface color prediction models rely on the fundamental assumption that (a) colorant surfaces are or can be assimilated to uniform surfaces and (b) that the attenuation of light by a print halftone expressed by its reflectance is a direct function of the surface coverages of the colorants. First color models, such as the Neugebauer model [6] relied on a linear color mixture model, i.e. they assumed that the light reflected by a halftone patch is the sum of the light reflected by the individual colorants, weighed by their respective surface coverages.

\subsection{The Neugebauer model}

In a 3D colorimetric space, for example the CIE-XYZ tri-stimulus color space, the Neugebauer color prediction model can be expressed for a cmy halftone composed of 8 colorants of known tri-stimulus values white $\mathbf{W}=\left(X_{W}, Y_{W}, Z_{W}\right)^{\mathrm{T}}$, cyan $\mathbf{C}=\left(X_{C}, Y_{C}, Z_{C}\right)^{\mathrm{T}}$, magenta $\mathbf{M}=\left(X_{M}, Y_{M}, Z_{M}\right)^{\mathrm{T}}$, yellow $\mathbf{Y}=\left(X_{Y}, Y_{Y}, Z_{Y}\right)^{\mathrm{T}}$, red $\mathbf{R}=\left(X_{R}, Y_{R}, Z_{R}\right)^{\mathrm{T}}$, green $\mathbf{G}=\left(X_{G}, Y_{G}, Z_{g}\right)^{\mathrm{T}}$, blue $\mathbf{B}=\left(\mathrm{X}_{\mathrm{G}}, \mathrm{Y}_{\mathrm{G}}, \mathrm{Z}_{\mathrm{G}}\right)^{\mathrm{T}}$, and chromatic black $\mathbf{K}=\left(\mathrm{X}_{\mathrm{G}}, \mathrm{Y}_{\mathrm{G}}, \mathrm{Z}_{\mathrm{G}}\right)^{\mathrm{T}}$. According to the Neugebauer model, the reflected color $\mathbf{H}=\left(X_{H}, Y_{H}, Z_{H}\right)^{\mathrm{T}}$ of such a color halftone is

$$
\mathbf{H}=a_{w} \mathbf{W}+a_{c} \mathbf{C}+a_{m} \mathbf{M}+a_{y} \mathbf{Y}+a_{r} \mathbf{R}+a_{g} \mathbf{G}+a_{b} \mathbf{B}+a_{k} \mathbf{K}
$$

By inserting the Demichel equations (3) for the colorant surface coverages $a_{i}$, we obtain as reflected color the result of a tri-linear interpolation within a unit cube whose vertices are the CIE-XYZ tri-stimulus values of the colorants. 


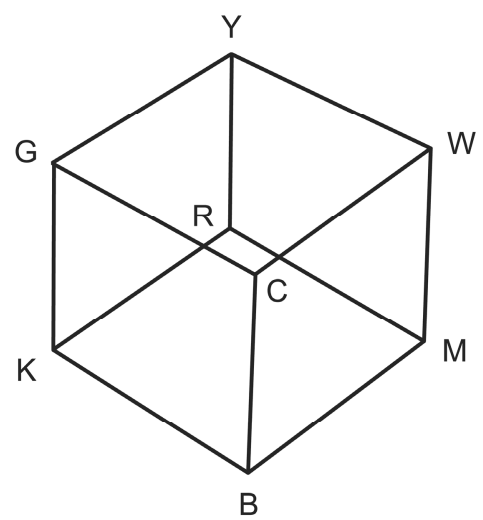

Figure 2: The cube formed by the colorants.

The Neugebauer model has been extended to work with spectral reflectances. With the spectral reflectances $R_{w}(\lambda), R_{c}(\lambda), R_{m}(\lambda), R_{y}(\lambda), R_{r}(\lambda), R_{g}(\lambda), R_{b}(\lambda), R_{k}(\lambda)$ of the respective colorants white (e.g. unprinted paper), cyan, magenta, yellow, red, green, blue and black, the halftone reflectance according to the spectral Neugebauer model is

$R(\lambda)=a_{\mathrm{w}} R_{w}(\lambda)+a_{\mathrm{c}} R_{c}(\lambda)+a_{\mathrm{m}} R_{m}(\lambda)+a_{\mathrm{y}} R_{y}(\lambda)+a_{\mathrm{r}} R_{r}(\lambda)+a_{\mathrm{g}} R_{g}(\lambda)+a_{\mathrm{b}} R_{b}(\lambda)+a_{\mathrm{k}} R_{k}(\lambda)$

The Murray-Davis model [7] is a special case of the Neugebauer model, where only two different colorants are present: one ink and paper white. Let us derive the Murray-Davis model (Figure 3). All symbols used in the present section refer either to a single wavelength or to the response of a sensor integrating the stimuli according to its spectral sensitivity over a certain wavelength range.

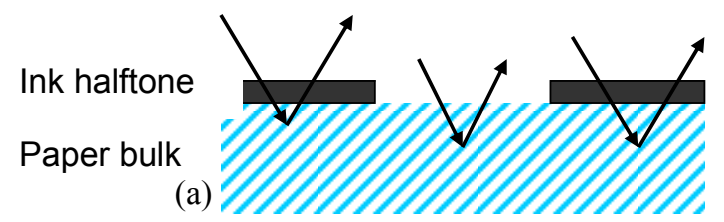

(b)

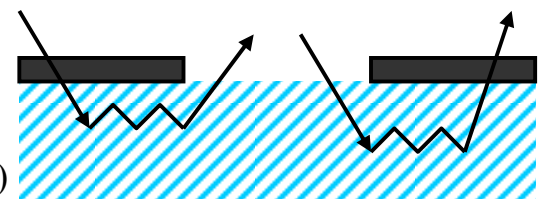

Figure 3: (a) Incident and exit locations of light are located within the same area (ink dot or unprinted paper), (b) due to lateral propagation of light, the incident and exit locations of light may be on different areas.

Let us assume that the incident light $I_{i n}$ strikes a black ink halftone of surface coverage $a_{k}$. A solid non-inked paper patch reflects a fraction $R_{p}$ of the incident light, where $R_{p}$ is the reflectance of paper. In a solid black ink patch, light travels through the ink layer (attenuation $T_{k}$ ) is reflected by the paper bulk and travels again through the ink layer before exiting patch. Light reflected by the paper is therefore attenuated twice by the ink layer and the corresponding 
reflectance is $R_{k}=T_{k}^{2} R_{p}$. Then, according to the Neugebauer equation, the overall reflectance is the weighted mean of the reflectances of the unprinted paper and the inked areas, with the weights being formed by their respective surface coverages. The reflected light $I_{\text {refl }}$ is

$$
I_{\text {refl }}=I_{\text {in }}\left[\left(1-a_{k}\right) R_{p}+a_{k} R_{k}\right]=I_{\text {in }}\left[\left(1-a_{k}\right) R_{p}+a_{k} T_{k}^{2} R_{p}\right]
$$

From the reflectance $R_{m}$ of the corresponding halftone, which can be measured, one can deduce the surface coverage $a_{k}$ of the ink. The corresponding formula, known as Murray-Davis formula is obtained as follows.

$$
\begin{aligned}
& R_{m}=I_{\text {refl }} / I_{\text {in }}=\left[\left(1-a_{k}\right) R_{p}+a_{k} R_{k}\right]=a_{k}\left[R_{k}-R_{p}\right]+R_{p} \\
& a_{k}=\frac{R_{m}-R_{p}}{R_{k}-R_{p}}=\frac{R_{p}-R_{m}}{R_{p}-R_{k}}
\end{aligned}
$$

Expressed in terms of optical density $D$, defined as $D=-\log _{10}(R)$, the reflectance becomes $R=10^{-D}$ and Eq. (9) becomes

$$
a_{k}=\frac{10^{-D_{p}}-10^{-D_{m}}}{10^{-D_{p}}-10^{-D_{k}}}=\frac{1-\left(10^{-D_{m}} / 10^{-D_{p}}\right)}{1-\left(10^{-D_{k}} / 10^{-D_{p}}\right)}=\frac{1-10^{-\left(D_{m}-D_{p}\right)}}{1-10^{-\left(D_{k}-D_{p}\right)}}
$$

where $D_{p}$ is the optical density of the paper, $D_{k}$ is the optical density of the solid ink patch, and $D_{m}$ the optical density of the halftone. Expression (10) is the Murray-Davis formula which is useful for estimating the dot size $a_{k}$ of a halftone with a densitometer giving directly the measured densities $D_{p}, D_{k}, D_{m}$, of respectively the paper, the full tone and the halftone.

The "Murray-Davis" dot size calculated according to Eq. (10) is widely used by offset printer operators in order to verify that the dot gain of the cyan, magenta, yellow and black ink dots printed on paper are within given bounds, see [8]. The dot gain is calculated as the obtained Murray-Davis dot size minus the nominal surface coverage that the printer is asked to print (also called control value). The densitometers used in the printing industry integrate the incoming light over the visible wavelength range according to sensitivities given by standards such as the DIN 16536-2 standard [9].

\subsection{Yule-Nielsen formula accounting for lateral propagation of light}

In the following sections, we consider light stimuli to be spectrally distributed. Wavelength dependent stimuli, reflectances or transmittances are functions of wavelength and named accordingly, e.g. $I(\lambda), R(\lambda)$ or $T(\lambda)$. Let us now assume that light propagates laterally within 
the print. If the lateral propagation of light is strong in respect to the halftone period, then the probability of a light ray to exit the print at a given ink location is independent from the position where the light ray entered the print. The attenuation of light entering the print and the attenuation of light exiting the print are therefore independent. The attenuation of light traversing the halftone ink layer from above is $\left[\left(1-a_{k}\right) \cdot 1+a_{k} T_{k}(\lambda)\right]$, where value " 1 " stands for the transmittance of the non-inked halftone area. Light is then reflected by the paper bulk with an attenuation $R_{p}(\lambda)$. It traverses again the ink layer and is attenuated by $\left[\left(1-a_{k}\right) 1+a_{k} T_{k}\right.$ $(\lambda)$. The global attenuation is the multiplication of these three attenuations.

$$
I_{r e f f}(\lambda)=I_{i n}(\lambda)\left[\left(1-a_{k}\right)+a_{k} T_{k}(\lambda)\right] R_{p}(\lambda)\left[\left(1-a_{k}\right)+a_{k} T_{k}(\lambda)\right]=I_{i n}(\lambda)\left[\left(1-a_{k}\right)+a_{k} T_{k}(\lambda)\right]^{2} R_{p}(\lambda)
$$

With the black reflectance $R_{k}(\lambda)=T_{k}(\lambda)^{2} R_{p}(\lambda)$, we obtain for the overall reflectance $R_{m}(\lambda)$ of the halftone

$$
\begin{aligned}
R_{m}(\lambda) & =I_{\text {refl }}(\lambda) / I_{\text {in }}(\lambda)=\left[\left(1-a_{k}\right)+a_{k} T_{k}(\lambda)\right]^{2} R_{p}(\lambda) \\
& =\left[\left(1-a_{k}\right)+a_{k}\left(R_{k}(\lambda) / R_{p}(\lambda)\right)^{1 / 2}\right]^{2} R_{p}(\lambda)=\left[\left(1-a_{k}\right) R_{p}(\lambda)^{1 / 2}+a_{k} R_{k}(\lambda)^{1 / 2}\right]^{2}
\end{aligned}
$$

We observe that the Yule-Nielsen [11] formula (12) creates a non-linear relation expressing the halftone reflectance as a function of the unprinted paper reflectance and the inked paper reflectance. Since $R_{m}(\lambda)^{1 / 2}=\left[\left(1-a_{k}\right) R_{p}(\lambda)^{1 / 2}+a_{k} R_{k}(\lambda)^{1 / 2}\right]$, we a have a linear relationship in the modified reflectance space $R(\lambda)^{1 / 2}$.

\section{Yule-Nielsen modified Spectral Neugebauer Model (YNSN)}

Since both the Murray-Davis and the Neugebauer model do not take into account the lateral propagation of light within the paper bulk nor the internal reflections (Fresnel reflections) at the paper-air interface, their predictions are not accurate [10]. Yule and Nielsen [11] expressed the reflected stimulus of a single ink halftone as a non-linear relationship between the reflected stimulus of paper and the reflected stimulus of the solid ink. This non-linear relationship, a generalization of Eq. (12), is a power function, whose exponent $n$ is fitted according to the stimulus responses of a limited set of measured halftone patches. Viggiano [12] applied the Yule-Nielsen relationship to the spectral Neugebauer equations, yielding the Yule-Nielsen modified Spectral Neugebauer (YNSN) model

$$
R(\lambda)=\left(\sum_{i} a_{i} \cdot R_{i}(\lambda)^{\frac{1}{n}}\right)^{n}
$$

where $R_{i}$ expresses the spectral reflectance of colorant $i, a_{i}$ expresses its effective surface coverage and the exponent $n$ is a scalar value generally higher than 1 . 
One can measure separately the reflectance $R_{i}$ of each solid colorant. However, in order to make accurate predictions, the effective surface coverage $a_{i}$ of each colorant $i$ of the halftone needs to be known as well as the optimal $n$-value associated with the considered combination of paper, inks, and color halftoning method. In general, the effective surface coverages $a_{i}$ of the colorants are obtained by mapping nominal to effective surface coverages. The mapping functions are established at model calibration time, by printing and measuring the reflectance of a number of uniform color calibration patches, at specific nominal halftone surface coverages such as 0.25 , 0.5 and 0.75 . The corresponding effective surface coverages are fitted, i.e. they are obtained by an optimization process minimizing a difference metric between measured reflectance and reflectance predicted according to the selected spectral prediction model (e.g. the YNSN model described by Eq.(13)). Detailed explanations about how to obtain effective surface coverages according an ink spreading model are given in Section 5.

Once the ink spreading model is associated with the spectral prediction model, the optimal $n$ value is obtained by trying to calibrate the spectral prediction model with different $n$-values, for example by traversing the set of all possible $n$-values between 1 and 20 in steps of 0.1 , and by selecting the $n$-value minimizing the sum of square differences between the predicted and the measured reflectance components of a subset of measured patches, e.g. the calibration patches.

\subsection{Cellular Yule-Nielsen modified spectral Neugebauer model (CYNSN)}

In order to provide a higher prediction accuracy, Heuberger et al. [13] proposed an approach where the full domain of the ink surface variations (Figure 2) is divided into sub-domains. The vertices of these subdomains, corresponding to specific nominal surface coverages of the inks, have known measured reflectances. Since the subdomains span a smaller subset of the color space compared with the original domain, the spectral prediction model relying on a function of subdomain vertex reflectances provides more accurate predictions. Subdomains may be created by dividing the CMY surface coverage unit cube into 8 subcubes (subdomains), formed by combinations of $0 \%, 50 \%$ and $100 \%$ surface coverages of the cyan, magenta and yellow inks (Figure $4 \mathrm{a}$ ). With such a subdivision, the number of primary reflectances increases from 8 to 27. Each subdomain, for example the one formed by ink coverages varying between $0 \%$ and $50 \%$, forms itself a spectral Neugebauer model formed by 8 of the 27 primary reflectances. In the case of 4 inks (CMYK), the same subdivision strategy can be applied in 4 dimensions. The 
initial 4D cube is subdivided into 16 subcubes, increasing the number of vertex reflectances from $2^{4}=16$ to $3^{4}=81$.

Balasubramanian [14] has shown that the cellular subdivision is also applicable to the YuleNielsen spectral Neugebauer model (name: CYNSN or simply "cellular Yule-Nielsen"). In order to achieve an even higher prediction accuracy, one can further subdivide each subcube into subsubcubes. However, for 3 inks, this additional subdivision step increases the number of measurements to $5^{3}=125$ and for 4 inks to $5^{4}=625$. There have been attempts to improve the cellular Yule-Nielsen model by an octtree like hierarchical subdivision of the initial cube until the desired prediction accuracy is reached within each leaf subcube [15].

(a)

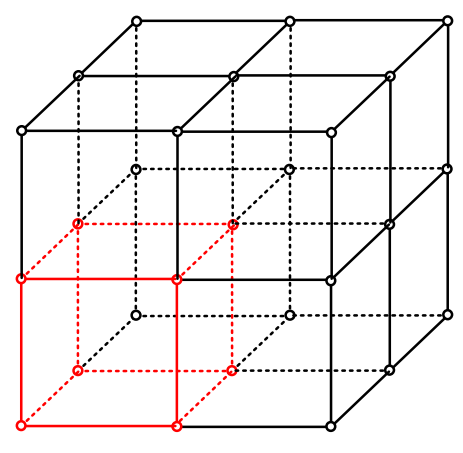

(b)

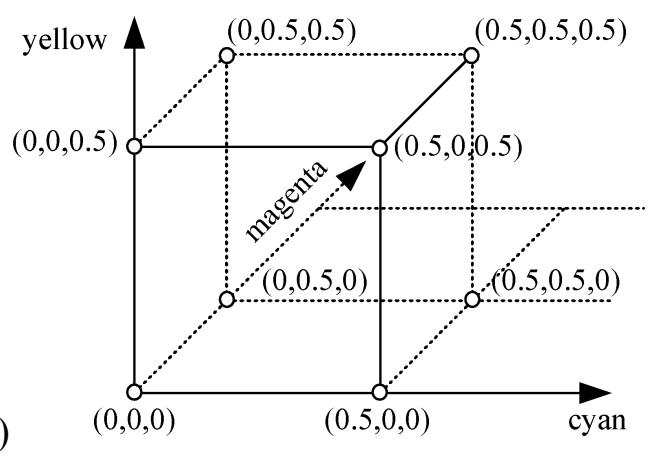

Figure 4: (a) Subdivision of the ink surface coverage space into subdomains produced by all combinations of $0 \%, 50 \%$ and $100 \%$ surface coverages of the three inks. (b) The subdomain with nominal surface coverages between $0 \%$ and $50 \%$. At the vertices of the subdomain cube, subdomain primary reflectances $R_{\mathrm{c}, \mathrm{m}, \mathrm{y}}(\lambda)$ have been measured.

Let us describe in detail the cellular Yule-Nielsen model with one subdivision level. The Neugebauer primaries, called subdomain primaries, are formed by surface coverages at all combinations of $0 \%, 50 \%$ and $100 \%$ surface coverages $\left(3^{3}=27\right.$ combinations). Figure $4 \mathrm{~b}$ illustrates a subdomain where the cyan, magenta and yellow ink surface coverages vary from 0 to 0.5 . Within each subdomain, the surface coverages are normalized. For an arbitrary cellular subdivision and with cyan, magenta and yellow ink surface coverages $c, m, y$ within a subdomain delimited by $c \in\left[c_{l}, c_{h}\right], m \in\left[m_{l}, m_{h}\right]$ and $y \in\left[y_{l}, y_{h}\right]$, the normalized $c^{\prime}, m^{\prime}, y^{\prime}$ ink coverages are

$$
c^{\prime}=\frac{c-c_{l}}{c_{h}-c_{l}} ; \quad m^{\prime}=\frac{m-m_{l}}{m_{h}-m_{l}} ; \quad y^{\prime}=\frac{y-y_{l}}{y_{h}-y_{l}}
$$


The predicted reflectance $R(\lambda)$ of a halftone of surface coverages $c \in\left[c_{l}, c_{h}\right], m \in\left[m_{l}, m_{h}\right], y \in$ $\left[y_{l}, y_{h}\right]$ is obtained by tri-linear interpolation of cube vertex reflectances

$$
\begin{aligned}
& R(\lambda)=\left(\left(1-c^{\prime}\right)\left(1-m^{\prime}\right)\left(1-y^{\prime}\right) R_{c l, m l, y l}(\lambda)^{1 / n}\right. \\
& +c^{\prime}\left(1-m^{\prime}\right)\left(1-y^{\prime}\right) R_{c h, m l, y l}(\lambda)^{1 / n}+\left(1-c^{\prime}\right) m^{\prime}\left(1-y^{\prime}\right) R_{c l, m h, y l}(\lambda)^{1 / n} \\
& +\left(1-c^{\prime}\right)\left(1-m^{\prime}\right) y^{\prime} R_{c l, m l, y h}(\lambda)^{1 / n}+\left(1-c^{\prime}\right) m^{\prime} y^{\prime} R_{c l, m h, y h}(\lambda)^{1 / n} \\
& +c^{\prime}\left(1-m^{\prime}\right) y^{\prime} R_{c h, m l, y h}(\lambda)^{1 / n}+c^{\prime} m^{\prime}\left(1-y^{\prime}\right) R_{c h, m h, y l}(\lambda)^{1 / n} \\
& \left.+c^{\prime} m^{\prime} y^{\prime} R_{c h, m h, y h}(\lambda)^{1 / n}\right)^{n}
\end{aligned}
$$

where $R_{c x, m x, y x}(\lambda)$ represents the measured spectral reflectance at surface coverages $(c, m, y)$ of the cyan, magenta and yellow inks, either at the low end of the interval $(x=l)$ or at the high end of the interval $(x=h)$.

\section{Accounting for ink spreading within the CYNSN model}

In order to improve the prediction accuracy, one may, instead of increasing the number of subdomains, account for ink spreading within each subdomain [16]. Within each subdomain, one ink spreading curve is associated to each ink and expresses the ink spreading behavior of its corresponding halftone dot. Since the physical dot gain of one ink within a subdomain does not depend strongly on the other ink surface coverages, we consider the ink spreading of each ink independently of the presence or absence of other superposed inks (see Sections 5 and 6). For each ink $i$ within each subdomain $j$, we define an ink spreading curve $f_{i, j}\left(u_{i, j}^{\prime}\right)$ mapping the normalized ink coverage $u_{i, j}^{\prime}$ to a normalized effective ink coverage $u_{i, j, e f f}^{\prime}$. The ink spreading curves may be obtained by printing halftones in one ink superposition condition, i.e. with one ink at a nominal surface coverage corresponding to the mid-range of the considered subdomain and the other inks at their lower bounds. For instance, the ink spreading curve for the cyan ink $(i=c)$ within the subdomain $j$ delimited by its low $(l)$ and high $(h)$ bounds $u_{i=c, j} \in\left[c_{j l}, c_{j h}\right], u_{i=m, j}$ $\in\left[m_{j l}, m_{j h}\right]$ and $u_{i=y, j} \in\left[y_{j l}, y_{j h}\right]$ is established by printing a halftone at cyan mid-range, magenta low and yellow low bound ink nominal surface coverages, i.e. a halftone at cyan $u_{i=c, j}=\left(c_{j l}+\right.$ $\left.c_{j h}\right) / 2$, at magenta $u_{i=m, j}=m_{j l}$ and at yellow $u_{i=y, j}=y_{j l}$ and by measuring its reflectance $R_{\left(c_{j l}+c_{j h}\right) / 2, m_{j l}, y_{j l}}(\lambda)$. Then, the mid-range cyan normalized effective surface coverage $q_{i=c, j}$ is fitted by minimizing the sum of square differences between the measured halftone reflectance components $R_{i=c, j}(\lambda)$ and the corresponding predicted reflectance

components $\hat{R}_{i=c, j}(\lambda)$. 


$$
\begin{aligned}
& R_{i=c, j}(\lambda) \quad=R_{\left(c_{j l}+c_{j h}\right) / 2, m_{j l}, y_{j l}}(\lambda) \\
& \hat{R}_{i=c, j}(\lambda) \quad=\left(q_{i=c, j} R_{\left.c_{j h}, m_{j l}, y_{j l}(\lambda)^{1 / n}+\left(1-q_{i=c, j}\right) R_{c_{j l}, m_{j l}, y_{j l}}\right)^{1 / n}}\right. \\
& q_{i=c, j}=\operatorname{argmin} \sum_{k}\left[R_{i=c, j}\left(\lambda_{k}\right)-\hat{R}_{i=c, j}\left(\lambda_{k}\right)\right]^{2}
\end{aligned}
$$

The other $q_{i=m, j}$, respectively $q_{i=y, j}$ mid-range normalized effective surface coverages are obtained in a similar manner, by exchanging in Eq. (16) the " $c$ " and the " $m$ " and respectively the " $c$ " and the " $y$ " indices. The fitted mid-range normalized effective surface coverage $q_{i, j}$ indicates the amount of ink spreading of ink $i$ within the subdomain $j$. The ink spreading curves $u_{i, j, e f f}^{\prime}=f_{i, j}\left(u_{i, j}^{\prime}\right)$ within the subdomain $j$ are obtained by quadratic interpolation between the points $(0,0),\left(0.5, q_{i, j}\right)$ and $(1,1)$, with $u_{i, j, e f f}^{\prime}=\left(2-4 \cdot q_{i, j}\right) u_{i, j}^{\prime 2}+\left(4 \cdot q_{i, j}-1\right) u_{i, j}^{\prime}$.

Computing the mid-range normalized effective surface coverages $q_{i=c, j}, q_{i=m, j}$ and $q_{i=y, j}$ with Eq. (16) requires for each subdomain $j$ three spectral reflectance measurements. For a 3-ink cellular model, we need 27 measurements of Neugebauer primaries and 24 measurements for fitting the 3 ink spreading curves with each subdomain.

In order to decrease the number of reflectance measurements to one per subdomain, we propose to jointly fit the mid-range normalized effective surface coverages $q_{i=c, j}, q_{i=m_{j} j}, q_{i=y, j}$ on a single halftone located at the center of the considered subdomain

$$
\begin{aligned}
& \hat{R}_{i=c e n t e r, j}(\lambda)=\left(\left(1-q_{i=c, j}\right)\left(1-q_{i=m, j}\right)\left(1-q_{i=y, j}\right) R_{c_{j l}, m_{j l}, y_{j l} l}(\lambda)^{1 / n}\right. \\
&+q_{i=c, j}\left(1-q_{i=m, j}\right)\left(1-q_{i=y, j}\right) R_{c_{j h}, m_{j l}, y_{j l}}(\lambda)^{1 / n} \\
&+\left(1-q_{i=c, j}\right) q_{i=m, j}\left(1-q_{i=y, j}\right) R_{c_{j l}, m_{j h}, y_{j l} l}(\lambda)^{1 / n} \\
&+\left(1-q_{i=c, j}\right)\left(1-q_{i=m, j}\right) q_{i=y, j} R_{c_{j l}, m_{j l}, y_{j h}}(\lambda)^{1 / n} \\
&+\left(1-q_{i=c, j}\right) q_{i=m, j} q_{i=y, j} R_{c_{j l}, m_{j h}, y_{j h}}(\lambda)^{1 / n} \\
&+q_{i=c, j}\left(1-q_{i=m, j}\right) q_{i=y, j} R_{c_{j h}, m_{j l}, y_{j h}}(\lambda)^{1 / n} \\
&+q_{i=c, j} q_{i=m, j}\left(1-q_{i=y, j}\right) R_{c_{j h}, m_{j h}, y_{j l}}(\lambda)^{1 / n} \\
&\left.+q_{i=c, j} q_{i=m, j} q_{i=y, j} R_{c_{j h}, m_{j h}, y_{j h}}(\lambda)^{1 / n}\right)^{n} \\
&\left\{q_{i=c, j}, q_{i=m, j}, q_{i=y, j}\right\}=\operatorname{argmin} \sum_{k}\left[R_{i=c e n t e r, j}\left(\lambda_{k}\right)-\hat{R}_{i=c e n t e r, j}\left(\lambda_{k}\right)\right]^{2}
\end{aligned}
$$




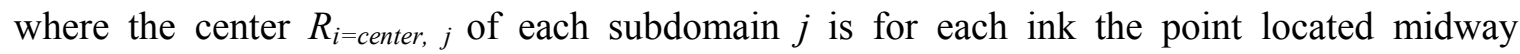
between the highest and lowest ink surface coverages:

$$
R_{i=\text { center }, j}(\lambda)=R_{\left(c_{j l}+c_{j h}\right) / 2,\left(m_{j l}+m_{j h}\right) / 2,\left(y_{j l}+y_{j h}\right) / 2}(\lambda)
$$

Figure 5 illustrates a cyan normalized dot gain curve for a CMY laser print, where the normalized dot gain is defined as $d_{i, j}\left(u_{i, j}^{\prime}\right)=f_{i, j}\left(u_{i, j}^{\prime}\right)-u_{i, j}^{\prime}$, here within the subdomain $j=1$ delimited by $c \in[0,0.5], m \in[0,0.5]$ and $y \in[0,0.5]$. In this example, the computed cyan midrange normalized effective surface coverage $q_{i=c, 1}$ for an optimal $n$-value of 14 calculated with Eq. (17) is equal to 0.61. It represents a normalized dot gain of 0.11 in the range [0,1] and therefore a real dot gain of 0.055 in the range [0,0.5]. The cellular Yule-Nielsen model prediction error for the considered halftone without taking into account the dot gain is $\Delta E_{94}=$ 3.60. Introducing the dot gain obtained by the fitted cyan mid-range normalized effective surface coverages $q_{i=c, j}$ decreases for this halftone the prediction error to $\Delta E_{94}=0.22$.

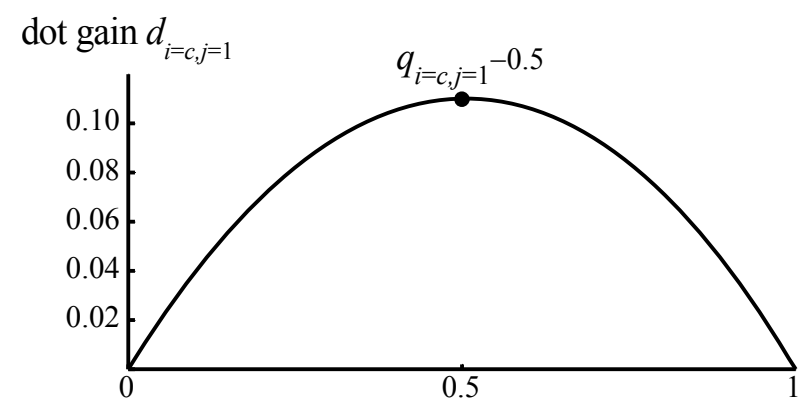

normalized nominal surface coverages

Figure 5: Cyan dot gain curve corresponding to the cyan ink spreading curve within the subdomain $c, m, y \in[0,0.5]$, for a CMY laser print (Brother 4000-HL) at a screen frequency of 120 lpi and using an optimal $n$-value of 14 .

As in the classical YNSN model, the optimal $n$-value is found by predicting for successive $n$ values with the ink spreading enhanced cellular Yule-Nielsen model the center of subdomain reflectances. The $n$-value yielding the minimal average prediction error is kept as the optimal $n$ value for the considered setup of printer, inks and paper.

The cellular Yule-Nielsen model accounting for ink spreading (IS-CYNSN) is illustrated in Figure 6. 


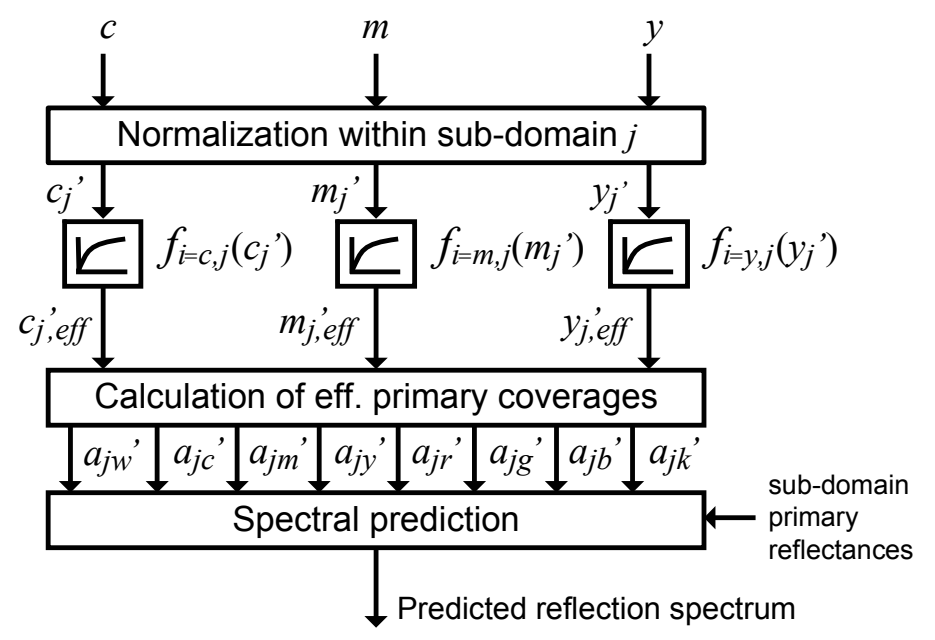

Figure 6: Cellular Yule-Nielsen model accounting for ink spreading (IS-CYNSN).

At model calibration time, the subdomain ink spreading curves $f_{i, j}\left(u_{i, j}^{\prime}\right)$ are established either by separately fitting the interpolation coefficients with Eq. (16) or by jointly fitting the interpolation coefficients with Eq. (17). At prediction time, nominal ink surface coverages of the considered halftone are normalized according to Eq. (14), the normalized effective ink surface coverages are deduced by making use of the corresponding ink spreading curves, the normalized effective surface coverages of the subdomain Neugebauer primary reflectances are calculated according to the Demichel equations (3) and the halftone reflection spectrum is predicted according to Eq. (15).

\subsection{The two-by-two dot centering model}

Ideally, a single printed dot is a perfect square of uniform ink thickness. In practice it looks more like an oval with a non-uniform thickness profile. Its exact size and thickness profile depend whether its neighborhood pixel locations are printed or not. Pappas [17] has developed an approach where the "grayscale" value of a printed dot is predicted according to whether its neighboring 8 pixels are printed or not. This prediction is then used to optimally halftone input grayscale images.

Wang [18] developed an improved method, where only a two-by-two pixel neighborhood is analyzed. The vertex of each square forming a printable pixel is the center of a two-by-two pixel neighborhood. An elementary square of the size of one pixel, called "two-by-two" square, is laid out across the considered 4 neighboring pixels (Figure 7). 


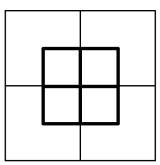

G0

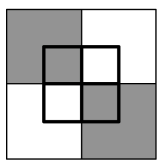

G4

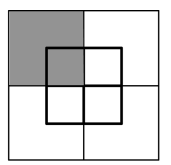

G1

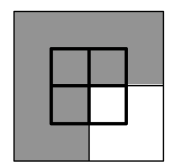

G5

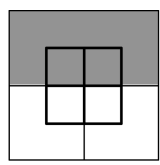

G2

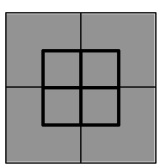

G6

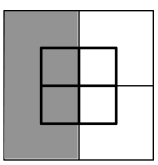

G3

(a)

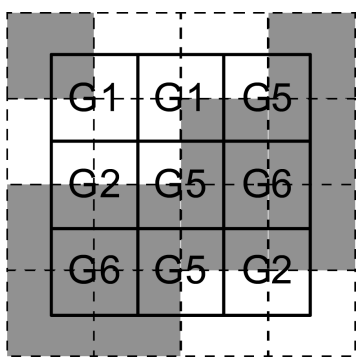

(b)

Figure 7: (a) Representative two by two square patterns G0 to G6 for single ink prints and (b) example of a 4 x 4 pixel halftone, overlaid by corresponding two-by-two square patterns.

Let us first consider a single ink print. Each two-by-two square is representative for the real printed layout at the junction of its 4 neighboring printable pixels. Since each pixel associated with a two-by-two square can be on or off, the two-by-two square can have $2^{4}=16$ different reflectance values. By taking into account horizontal, vertical and central symmetries, the number of representative reflectance values of two-by-two squares that need to be differentiated can be reduced to 7 (Figure 7). These 7 different reflectances can be learned by measuring the reflectances of tiles, each one composed of only one of these representative twoby-two patterns, repeated over a large surface. Figure 8 gives the calibration tiles associated with the corresponding representative two-by-two squares.

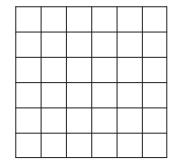

G0

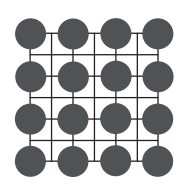

G1

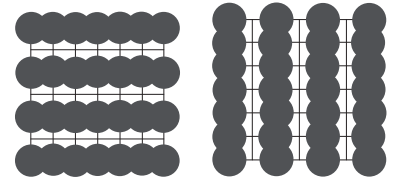

G2

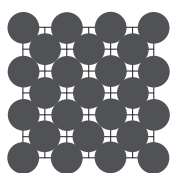

G4

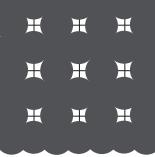

G5

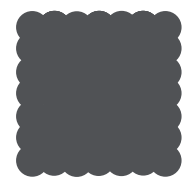

G6

Figure 8: Calibration tiles associated to the corresponding representative two-by-two squares.

In order to predict the reflectance of any input halftone or pattern image with the learned representative two-by-two patterns, one traverses the input image, by moving a two-by-two unit square from pixel square vertex to pixel square vertex, finding its associated representative pattern and creating an output bitmap formed of two-by-two unit squares labeled as G0 to G6 (Figure 7). The input image is now segmented into two-by-two labeled unit squares. Since the "microscopic" reflectance of each two-by-two labeled unit square is known, the "macroscopic" 
reflectance of a larger tile can be computed, for example a tile covering the area of a halftone. The reflectance of an image area formed by an assembly of two-by-two unit square reflectances may be calculated with the Yule-Nielsen modified spectral Neugebauer model (YNSN), according to Equation (19), where $i_{\mathrm{m}}$ is the number of occurrences of representative two-bytwo pattern $G_{m}$ in the considered area and $R_{\mathrm{m}}(\lambda)$ is its corresponding measured reflectance spectrum.

$$
R^{1 / n}(\lambda)=\sum_{m=0}^{6} i_{m} \cdot R_{m}{ }^{1 / n}(\lambda) / \sum_{m=0}^{6} i_{m}
$$

The two-by-two centering model can be extended to multiple ink prints [19]. For dots within a two-by-two tile being printed with more than the black and white colors, the number of patterns that can occur within a two-by-two tile increases significantly. The number of possible arrangements for the four printed dots of a two-by-two tile and for $N$ solid colorants is $N^{4}$. In the case of a CMY print ( 8 colorants), there are $8^{4}=4096$ possible colors arrangements within a single two-by-two tile. If we remove both the horizontal and the vertical symmetries, $P(8)=1072$ independent patterns remain. In the case of 4 inks, there are 16 solid colorants and $16^{4}=65536$ possible arrangements. By removing horizontal and vertical symmetries, $P(16)=16576$ independent patterns remain. Let us rewrite Equation (19) for the case of color predictions with $N$ solid colorants

$$
R^{1 / n}(\lambda)=\sum_{m=0}^{P(N)-1} i_{m} \cdot R_{m}^{1 / n}(\lambda) / \sum_{m=0}^{P(N)-1} i_{m}
$$

For $N$ solid colorants, the number of remaining patterns $P(N)$ remaining after considering horizontal and vertical symmetries can be calculated as follows. We first consider the total number of arrangement $Q(N)=N^{4}$. In the following arrangements, a horizontal or vertical symmetry does not induce two different patterns: (a) same color on all 4 pixels, (b) in respect to horizontal symmetry, same color on the two horizontal neighboring pixels and (c) in respect to vertical symmetry, same color on two neighboring vertical pixels. Table 1 gives the number of arrangements for these cases, both for 3-ink and 4-ink prints and shows how to compute the number of representative patterns. 
Table 1. Calculation of symmetry reduced representative patterns, where $c_{1}$ represents one colorant and $c_{2}$ represents another colorant.

\begin{tabular}{|c|c|c|c|}
\hline & & 3 inks ( $N=8$ colorants $)$ & 4 inks ( $N=16$ colorants) \\
\hline Uniform color of all 4 pixels & $\begin{array}{ll}c_{1} & c_{1} \\
c_{1} & c_{1}\end{array}$ & 8 arrangements & 16 arrangements \\
\hline $\begin{array}{l}\text { Horizontal neighbors of } \\
\text { uniform color, but vertical } \\
\text { neighbors of different colors }\end{array}$ & $\begin{array}{ll}c_{1} & c_{1} \\
c_{2} & c_{2}\end{array}$ & $\begin{array}{l}\text { Select } 2 \text { from } 8 \text { colorants } \\
\left(\begin{array}{l}8 \\
2\end{array}\right)=28 \text { arrangements }\end{array}$ & $\begin{array}{l}\text { Select } 2 \text { from } 16 \text { colorants } \\
\left(\begin{array}{c}16 \\
2\end{array}\right)=120 \text { arrangements }\end{array}$ \\
\hline $\begin{array}{l}\text { Vertical neighbors of } \\
\text { uniform color, but horizontal } \\
\text { neighbors of different colors }\end{array}$ & $\begin{array}{ll}c_{1} & c_{2} \\
c_{1} & c_{2}\end{array}$ & $\begin{array}{l}\text { Select } 2 \text { from } 8 \text { colorants } \\
\left(\begin{array}{l}8 \\
2\end{array}\right)=28 \text { arrangements }\end{array}$ & $\begin{array}{l}\text { Select } 2 \text { from } 16 \text { colorants } \\
\left(\begin{array}{c}16 \\
2\end{array}\right)=120 \text { arrangements }\end{array}$ \\
\hline $\begin{array}{l}\text { Total number of non- } \\
\text { symmetric arrangements }\end{array}$ & & $8+28+28=64$ & $16+120+120=256$ \\
\hline $\begin{array}{l}\text { Symmetry reduced number of } \\
\text { representative patterns } P(N)\end{array}$ & & $\frac{4096-64}{4}+64=1072$ & $\frac{65536-256}{4}+256=16576$ \\
\hline
\end{tabular}

One may significantly reduce the number of calibration tile measurements by predicting the reflectances of the large majority of representative two-by-two patterns. The predictions rely on a small subset of measured two-by-two pattern tiles [20], typically $10 \%$ of the total number of representative pattern tiles.

The present two-by-two spectral prediction method is to some extent halftone independent. After measurement of the reflection spectra of all representative two-by-two patterns, any halftone print can by tiled into representative two-by-two patterns and the reflectance of specific areas can be predicted thanks to Eqs. (19) or (20), where the $n$-value is generally similar for single ink or multiple ink halftones and can therefore be learned from a set of single ink halftones, printed on paper and on different solid colorants, for example the halftones shown in Section 6, Figure 18, rows C and F or for increased accuracy, rows B, C, D, E, F and G.

Experience has shown that for different halftone screen frequencies, the optimal $n$-values used for the assembly of representative two by two square patterns according to Eqs. (19) or (20) may vary. Since the representative two by two square patterns already incorporate the optical dot gain induced by its 4 neighboring pixels, these optimal $n$-values are generally smaller than 
the ones used for predicting the reflectance of the same halftones with the Yule-Nielsen modified spectral Neugebauer model, see Section 3.2.

\section{PHYSICALLY INSPIRED MODELS}

In order to smoothly introduce physically inspired models, we first establish the Clapper-Yule model [21] for the reflectance of halftone ink layers laid out on a Lambertian reflecting substrate. In the Clapper-Yule model, a wavelength dependent ink transmittance is considered, that is independent of the orientation of the incident illumination and of the orientation of the capturing device. We describe the Clapper-Yule model both for the integrated sphere and the $\left(\theta^{\text {in }}: \theta^{\text {out }}\right)$ measurement geometries. We also briefly discuss extensions of the Clapper-Yule model applicable to mid-range and to low screen frequencies.

The Williams-Clapper model based models [22] differentiate themselves from the ClapperYule model by the fact that the transmittance of the ink layers varies as a function of the orientation in which the light traverses the ink layers.

There are a number of additional useful physically based prediction models. Some of them are described in this book. Let us mention hdi053 initially published in [23], [24], [25], hdi052, hdi054 published in [26], hdi055, initially published in [27], [28], [29], [30], the KubelkaMunk model, see hdi062, Section 7 initially published in [31], [32] as well as ink penetration models [33], [25].

In order to introduce the Clapper-Yule and Williams-Clapper based models, let us briefly define the nomenclature. We consider a solid non-diffusing colorant layer of refractive index $n_{2}$, having a transmittance $t(\lambda)$, in contact with a Lambertian substrate of intrinsic reflectance $r_{g}(\lambda)$ on the one side, and in contact with air on the other side. Instead of a solid colorant layer, one may have a halftone layer formed by several colorants of respective transmittances $t_{j}(\lambda)$. Hereinafter, the term colored layer indicates a solid ink or a halftone ink layer. The substrate and the colored layer are assumed to have the same refractive index. The interface between the colored layer and the air is a planar interface. The refractive index of air is $n_{1}=1$. The relative refractive indices of the interfaces $n_{2} / n_{1}$ (layer to air) and $n_{1} / n_{2}$ (air to layer) are respectively $n_{2}$ 
and $1 / n_{2}$. In respect to the interface, index 1 indicates the air side and index 2 indicates the layer side.

In order to model the global reflectance of light from a print, we consider three different attenuations: (a) the attenuation $T_{\text {in }}$ of the incident light reaching the substrate, (b) the attenuation of light $T_{m}$ due to multiple reflections and attenuations between the diffusing substrate and the colored layer-air interface and (c) the attenuation of light $T_{\text {out }}$ due to the exit of light across the colored layer-air interface.

In the present section, Fresnel reflections and transmissions are assumed to be wavelength independent. But all transmittances, reflectances and reflectance factors are wavelength dependent. For ease of presentation, this wavelength dependence is not always marked explicitly in the equations. It can however be clearly deduced from the context.

\subsection{The Clapper-Yule reflectance prediction model for color halftones}

The Clapper-Yule model [21] was initially proposed for a single ink halftone and an integrating sphere measurement geometry. It accounts for the following physical phenomena: specular reflection of the incident light at the print-air interface, attenuation of light when traversing the halftone ink layer, scattering and reflection within the paper bulk, Fresnel reflection at the print-air interface yielding multiple internal reflections and Fresnel transmission of the exiting light (Figure 9). We present first the case of the integrating sphere light capturing geometry, and then discuss the case of a $\theta^{\text {in }}: \theta^{\text {out }}$ geometry. We start first with a single ink halftone and then generalize to multi-ink halftones.

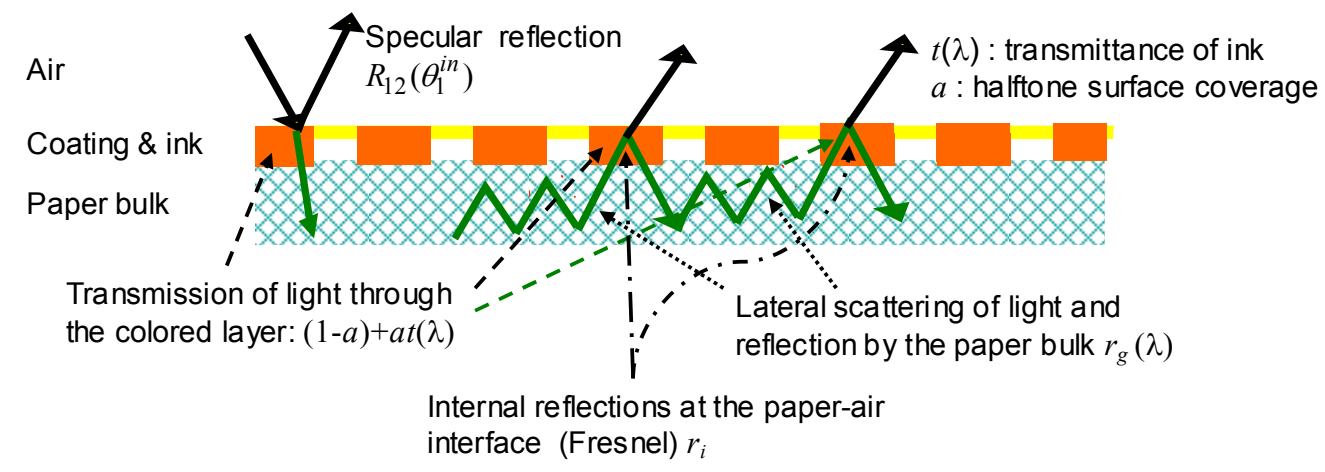

Figure 9: Phenomena accounted for by the Clapper-Yule model. 
Clapper-Yule model for single ink halftones (geometry $\theta_{1}^{\text {in }}: d$ )

Collimated incident light of irradiance $E_{i}$ hits the halftone print, part of it is specularly reflected according to the Fresnel reflection factor $R_{12}\left(\theta_{1}^{i n}\right)$ and the other part $1-R_{12}\left(\theta_{1}^{i n}\right)$ enters the print. The part entering the print traverses either an inked area $a$ of transmittance $t(\lambda)$, or an unprinted area $(1-a)$ of transmittance 1 . When reaching the diffusing substrate (paper bulk), light has been attenuated by the colored layer by the factor $(1-a)+a t(\lambda)$.

The diffusing substrate reflects over the whole hemisphere an irradiance $E_{0}(\lambda)=\left(1-R_{12}\left(\theta_{1}^{i n}\right)\right) \cdot(1-a+a t(\lambda)) \cdot r_{g}(\lambda) \cdot E_{i}(\lambda)$ which is the irradiance having crossed the halftone layer, attenuated by the substrate reflectance $r_{g}(\lambda)$.

The diffuse irradiance $E_{0}$ reflected from the substrate is assumed to be Lambertian, and can therefore be expressed as an orientation independent radiance $L_{0}=E_{0} / \pi$. Let us calculate the internal Fresnel reflectance $r_{i}$ at the halftone layer-air interface by integrating the reflected irradiance elements over the whole hemisphere (Figure 10). At each angular orientation $\theta$, an irradiance element $d E=L \cos \theta \mathrm{d} \omega=\left(E_{0} / \pi\right) \cos \theta d \omega$ is emitted by the substrate (Lambertian reflector) within an infinitesimal solid angle $\mathrm{d} \omega$.

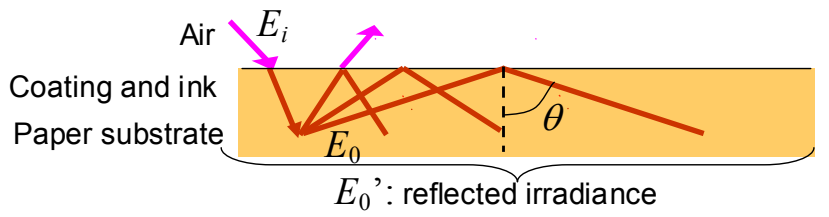

Figure 10. Irradiance elements reflected by the paper bulk.

A fraction $R_{21}(\theta)$, given by the Fresnel formulae, of each irradiance element of orientation $\theta$ located within an elementary solid angle $\mathrm{d} \omega$ reaching the print-air interface is reflected. One obtains the total amount of reflected irradiance by integrating over the whole hemisphere irradiance elements individually reflected at the print-air interface within infinitesimal angle $d \omega$ (Figure 11). 


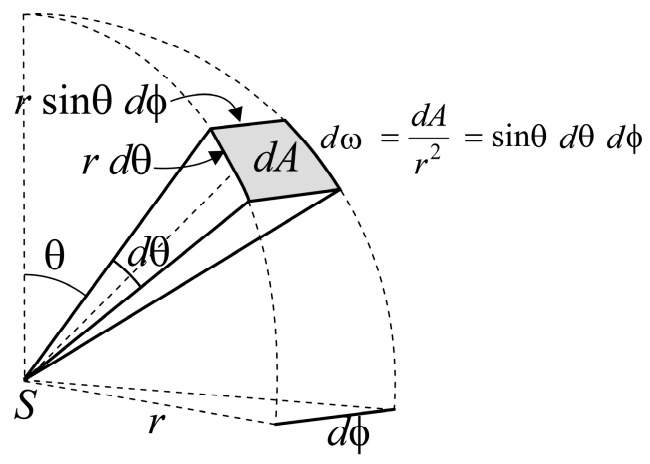

Figure 11. Infinitesimal solid angle $d \omega=\sin \theta \mathrm{d} \theta \mathrm{d} \phi$

We obtain for the irradiance $E_{0}{ }^{\prime}$ reflected at the print-air interface

$$
E_{0}{ }^{\prime}(\lambda)=\int \frac{E_{0}(\lambda)}{\pi} \cdot R_{21}(\theta) \cos \theta \cdot d \omega=\int_{0}^{2 \pi} \int_{0}^{\pi / 2} \frac{E_{0}(\lambda)}{\pi} \cdot R_{21}(\theta) \cos \theta \sin \theta d \theta d \varphi=E_{0}(\lambda) \int_{0}^{\pi / 2} R_{21}(\theta) \sin 2 \theta d \theta
$$

By dividing the total reflected irradiance $E_{0}{ }^{\prime}$ by the irradiance $E_{0}$ incident on the print-air interface, we obtain the average reflection $r_{i}$ of Lambertian light at the print-air interface

$$
r_{i}=\int_{0}^{\pi / 2} R_{21}(\theta) \sin 2 \theta d \theta
$$

At the print-air interface (internal print interface), for a print with an assumed index of refraction of $n=1.5$, Eq. (22) comprising Fresnel coefficient $R_{21}(\theta)$ yields the overall reflectivity $r_{i}=0.596$ [34], see also hdi062, Section 3.4.

This diffuse irradiance $E_{0}(\lambda)=\left(1-R_{12}\left(\theta_{1}^{i n}\right)\right)(1-a+a \cdot t(\lambda)) \cdot r_{g}(\lambda) \cdot E_{i}(\lambda)$ is emitted from the substrate towards the colored halftone layer, traverses the halftone layer, is partially reflected at the halftone layer - air interface, traverses again the halftone layer, reaches the substrate and is reflected from it for the second time. The attenuation of this single reflection cycle comprises the attenuation of traversing twice the halftone layer $\left(1-a+a t^{2}\right)$, the Fresnel reflection at the halftone layer - air interface $r_{i}$, and the diffusion-reflection by the substrate $r_{g}(\lambda)$.

After one full reflection cycle, we obtain the attenuated irradiance $E_{1}=\left(1-a+a t^{2}\right) r_{i} r_{g} E_{0}$. At the $k^{\text {th }}$ full reflection cycle, we have the attenuated irradiance $E_{\mathrm{k}}=\left[\left(1-a+a t^{2}\right) r_{i} r_{g}\right]^{k} E_{0}$. By summing these attenuated irradiance components, we obtain the total irradiance $E_{\mathrm{S}}$ emerging from the substrate 


$$
E_{S}(\lambda)=\left(\sum_{k=0}^{\infty}\left(r_{i} \cdot r_{g}(\lambda) \cdot\left(1-a+a \cdot t(\lambda)^{2}\right)\right)^{k}\right) E_{0}(\lambda)
$$

which is a geometric series and can be expressed by

$$
E_{S}(\lambda)=\frac{1}{1-r_{g}(\lambda) r_{i}\left(1-a+a \cdot t(\lambda)^{2}\right)} E_{0}(\lambda)=\frac{\left(1-R_{12}\left(\theta_{1}^{i n}\right)\right)(1-a+a t(\lambda)) \cdot r_{g}(\lambda)}{1-r_{g}(\lambda) r_{i}\left(1-a+a t(\lambda)^{2}\right)} \cdot E_{i}(\lambda)
$$

Part $\left(1-r_{i}\right)$ of irradiance $E_{s}$ traverses the print-air interface and exits from the print after having been attenuated by $(1-a+a t)$ through the halftone layer. The exiting irradiance captured by an integrated sphere is

$$
E_{\text {out }}(\lambda)=\frac{\left(1-R_{12}\left(\theta_{1}^{i n}\right)\right)(1-a+a \cdot t(\lambda))^{2} \cdot r_{g}(\lambda) \cdot\left(1-r_{i}\right)}{1-r_{g}(\lambda) r_{i}\left(1-a+a \cdot t(\lambda)^{2}\right)} \cdot E_{i}(\lambda)
$$

The Clapper-Yule model reflectance obtained with a $\left(\theta^{\text {in }}: d\right)$ measurement geometry is

$$
R(\lambda)=\frac{E_{\text {out }}(\lambda)}{E_{\text {in }}(\lambda)}=K \cdot R_{12}\left(\theta_{1}^{\text {in }}\right)+\frac{\left(1-R_{12}\left(\theta_{1}^{\text {in }}\right)\right)(1-a+a \cdot t(\lambda))^{2} \cdot r_{g}(\lambda) \cdot\left(1-r_{i}\right)}{1-r_{g}(\lambda) r_{i}\left(1-a+a \cdot t(\lambda)^{2}\right)}
$$

where $K$ indicates the portion of specularly reflected light captured by the integrated sphere. If the specular reflection is discarded, $K$ is set to zero. Note that Eq. (26) also represents the reflectance factor of a halftone print measured by an integrating sphere using a perfect white diffuser as reference.

\section{Calculating the paper reflectance and ink transmittance parameters}

The internal paper reflectance $r_{g}(\lambda)$ can be deduced from Eq. (26),

$$
r_{g}(\lambda)=\frac{R_{p}(\lambda)-K \cdot r_{s}}{1+(1-K) \cdot r_{i} \cdot r_{s}+r_{i} \cdot R_{p}(\lambda)-r_{s}-r_{i}}
$$

where $R_{p}(\lambda)$ is the measured reflectance of the paper, $r_{s}=R_{12}\left(\theta_{1}^{i n}\right)$ is the specular component at the air-print interface (for a print of index of refraction $n=1.5, r_{i}=0.596$ and at $\theta_{1}^{i n}=45^{\circ}, r_{s}=$ $0.050)$.

Once $r_{g}(\lambda)$ is calculated, the ink transmittance $t_{j}(\lambda)$ of colorant $j$ is deduced from Eq. (26)

$$
t_{j}(\lambda)=\sqrt{\frac{R_{j}(\lambda)-K \cdot r_{s}}{r_{g}(\lambda) \cdot r_{i} \cdot\left(R_{j}(\lambda)-K \cdot r_{s}\right)+r_{g}(\lambda) \cdot\left(1-r_{i}\right) \cdot\left(1-r_{s}\right)}}
$$

where $R_{j}(\lambda)$ is the measured reflectance of the solid ink patch. 
Expressing the reflectance as a composition of input, internal and exit attenuations

One may verify that the reflectance can be expressed by an input term $T_{i n}(\lambda)$ indicating the attenuation of the incoming light until it reaches the substrate, a term $R_{m}(\lambda)$ giving the internal reflectance of light within the print and a term $T_{\text {out }}(\lambda)$ giving the attenuation of light exiting the print. In the present case, the input attenuation is formed by the Fresnel transmittance at the interface air- halftone layer and by the attenuation of the halftone layer

$$
T_{\text {in }}(\lambda)=\left(1-R_{12}\left(\theta_{1}^{\text {in }}\right)\right)(1-a+a \cdot t(\lambda))
$$

The internal reflectance of light within the print including the multiple reflections between the substrate and the print-air interface is

$$
R_{m}(\lambda)=\frac{r_{g}(\lambda)}{1-r_{g}(\lambda) r_{i}\left(1-a+a \cdot t(\lambda)^{2}\right)}
$$

and the attenuation of light exiting the print is

$$
T_{\text {out }}(\lambda)=(1-a+a \cdot t(\lambda))\left(1-r_{i}\right)
$$

With formula (26), (29), (30) and (31), one can verify that indeed, if we assume that the measuring instrument discards the specular reflection, $R=T_{\text {in }} R_{m} T_{\text {out }}$. Input and output attenuations depend respectively on the illumination and observation geometries. Since the substrate is assumed to be strongly scattering, i.e. Lambertian, $R_{m}$ is independent of the illumination and measuring geometries.

\section{Assumptions underlying the Clapper-Yule model}

Note that we have completely separated the attenuation of the halftone layer for the incident light (Eq. (29)) and the attenuation of the halftone layer for the exiting light (Eq. (31)). For the exiting light, the probability to exit from an ink dot or from unprinted paper is proportional to their surface coverages, i.e. the location from which a light beam arrives (printed or unprinted area) has no influence on its exit location. This means that the lateral propagation of light within the print due to scattering and multiple reflections is high in respect to the halftone screen period. Experience shows that the Clapper-Yule model works well at screen frequencies above 1201pi. A second important assumption is that the substrate is strongly diffusing, i.e. it can be modeled by a Lambertian reflector. 


\section{The Saunderson correction as a special case of the Clapper-Yule model}

A further interesting point concerns the case of a solid colored layer of transmittance $t(\lambda)$. By setting the surface coverage $a=1$, we obtain for the reflectance $R_{\text {solid }}$ of a solid colored layer on a diffusing substrate

$$
R_{\text {solid }}(\lambda)=K \cdot R_{12}\left(\theta_{1}^{i n}\right)+\frac{\left(1-R_{12}\left(\theta_{1}^{i n}\right)\right) t(\lambda)^{2} r_{g}(\lambda)\left(1-r_{i}\right)}{1-r_{g}(\lambda) r_{i} t(\lambda)^{2}}
$$

The Saunderson correction introduces a correction factor in respect to the intrinsic reflectance in order to account for the internal Fresnel reflections and refractions at the boundaries between the considered diffusing layer and the air [35]. Knowing the intrinsic reflectance $\rho(\lambda)$ of the diffusing layer without interface, the Saunderson formula gives the reflectance $R(\lambda)$ accounting for the interface

$$
R(\lambda)=K \cdot r_{s}+\frac{\left(1-r_{s}\right) \cdot\left(1-r_{i}\right) \cdot \rho(\lambda)}{1-r_{i} \cdot \rho(\lambda)}
$$

where $r_{s}$ is the specular Fresnel reflection at the air-layer interface and $r_{i}$ is the internal Fresnel reflection at the layer-air interface given by Eq. (22).

The specular reflection component is $r_{s}=R_{12}\left(\theta_{1}^{i n}\right)$ and by equating $\rho(\lambda)=t(\lambda)^{2} r_{g}(\lambda)$ we observe that the Clapper-Yule equation for a solid colored layer given by Eq. (32) is identical to the Saunderson correction of that colored layer on a diffusing substrate, modeled by light passing twice through the solid colored layer (attenuation $\left.t(\lambda)^{2}\right)$ and being reflected once by the diffusing substrate (attenuation $r_{g}(\lambda)$ ).

Clapper-Yule model for multiple ink halftone (geometry $\left.\theta_{1}^{\text {in }}: d\right)$

Multi-chromatic halftones are formed by the colorants (Neugebauer primaries) comprising the single inks and their superpositions. The surface coverages $a_{i}$ of the colorants are derived from the surface coverages of the inks. In the case of three independently printed ink layers, Eqs. (3) are applicable.

We can extend the reflectance of a halftone made of a single ink halftone and the unprinted paper to a halftone comprising a certain number of colorants, e.g. 3 inks yielding 8 colorants or 4 inks yielding 16 colorants. In the case of a single ink halftone, the halftone surface is formed 
by the printed dots of surface coverage $a$ and the unprinted paper of surface coverage $(1-a)$. In the case of $m$ colorants, the halftone surface is formed by the juxtaposition of the colorants with respective surface coverages $a_{1}, a_{2}, \ldots a_{m}$ (Figure $16 \mathrm{~b}$ ). The total surface is 1 , i.e. $\sum a_{j}=1$. The input attenuation becomes

$$
T_{i n}=\left(1-R_{12}\left(\theta_{1}^{i n}\right)\right) \cdot \sum_{j} a_{j} t_{j}(\lambda)
$$

The internal attenuation becomes

$$
R_{m}=\frac{r_{g}}{1-r_{g}(\lambda) r_{i} \sum_{j} a_{j} t_{j}(\lambda)^{2}}
$$

and the exit term becomes

$$
T_{\text {out }}=\left(1-r_{i}\right) \sum_{j} a_{j} t_{j}(\lambda)
$$

The global Clapper-Yule model for multi ink halftone prints is

$$
R=K \cdot R_{12}\left(\theta_{1}^{i n}\right)+\frac{\left(1-R_{12}\left(\theta_{1}^{i n}\right)\right)\left(\sum_{j} a_{j} t_{j}(\lambda)\right)^{2} \cdot r_{g}(\lambda) \cdot\left(1-r_{i}\right)}{1-r_{g}(\lambda) r_{i}\left(\sum_{j} a_{j} t_{j}(\lambda)^{2}\right)}
$$

The internal paper reflectance $r_{g}(\lambda)$ and the colorant transmittances $t_{j}(\lambda)$ ) are deduced according to equations (27) and (28) from measurements of respectively the paper reflectance $R_{w}(\lambda)$ and the solid colorant patch reflectances $R_{j}(\lambda)$.

Clapper-Yule model, with measurements according to a $\left(\theta_{1}^{\text {in }}: \theta_{1}^{\text {out }}\right)$ measuring geometry

When measuring the exiting radiance at a given output angle instead of measuring the whole reflected irradiance over the hemisphere by an integrated sphere, only the exit term $T_{\text {out }}$ changes. The total irradiance $E_{\mathrm{S}}(\lambda)$ emerging from the substrate is defined by Eq. (24). Since emission from paper is considered to be Lambertian, the corresponding radiance emitted from the substrate for a single ink halftone is

$$
L_{S}(\lambda)=\frac{E_{S}(\lambda)}{\pi}=\frac{\left(1-R_{12}\left(\theta_{1}^{i n}\right)\right)(1-a+a \cdot t(\lambda)) \cdot r_{g}(\lambda)}{1-r_{g}(\lambda) r_{i}\left(1-a+a \cdot t(\lambda)^{2}\right)} \cdot \frac{E_{i}(\lambda)}{\pi}
$$


With Eq. (44) from Chapter hdi062, the radiance $L$ emerging from the print is radiance $L_{s}$ attenuated by passing once through the ink layer (attenuation: 1-a+at) and traversing the printair interface with an attenuation $\left(1-R_{21}\left(\theta_{2}^{\text {out }}\right)\right) / n_{2}^{2}=\left(1-R_{12}\left(\theta_{1}^{\text {out }}\right)\right) / n_{2}^{2}$, where according to Snell's law, $\theta_{2}^{\text {out }}=\arcsin \left(n_{1} \sin \theta_{1}^{\text {out }} / n_{2}\right)$. The exit attenuation term becomes

$$
T_{\text {out }}(\lambda)=\frac{1-R_{21}\left(\theta_{2}\right)}{n_{2}^{2}}(1-a+a \cdot t(\lambda))=\frac{1-R_{12}\left(\theta_{1}\right)}{n_{2}^{2}}(1-a+a \cdot t(\lambda))
$$

We obtain for the exit angle dependent radiance

$$
L_{\text {out }}\left(\theta_{1}^{\text {out }}, \lambda\right)=\frac{\left(1-R_{12}\left(\theta_{1}^{\text {in }}\right)\right)(1-a+a \cdot t(\lambda))^{2} \cdot r_{g}(\lambda)}{1-r_{g}(\lambda) r_{i}\left(1-a+a \cdot t(\lambda)^{2}\right)} \cdot \frac{1-R_{12}\left(\theta_{1}^{\text {out }}\right)}{n_{2}^{2}} \cdot \frac{E_{i}(\lambda)}{\pi}
$$

We calculate the radiance factor $R_{f a c}(\lambda)$ as the ratio of the exit radiance at angle $\theta_{1}^{\text {out }}$ and of the radiance $E_{i}(\lambda) / \pi$ reflected by a perfectly reflecting Lambertian reflector. We obtain

$$
R_{f a c}\left(\theta_{1}^{\text {out }}, \lambda\right)=\frac{L_{\text {out }}\left(\theta_{1}^{\text {out }}\right)}{E_{i} / \pi}=\frac{\left(1-R_{12}\left(\theta_{1}^{\text {in }}\right)(1-a+a t(\lambda))^{2} \cdot r_{g}(\lambda)\right.}{1-r_{g}(\lambda) r_{i}\left(1-a+a t(\lambda)^{2}\right)} \cdot \frac{1-R_{12}\left(\theta_{1}^{\text {out }}\right)}{n_{2}^{2}}
$$

When extending to multi-ink halftones, in a similar manner as in the previous section, we obtain for the Clapper-Yule radiance factor

$$
R_{f a c}(\lambda)=\frac{\left(1-R_{12}\left(\theta_{1}^{\text {in }}\right)\right)\left(\sum_{j} a_{j} t_{j}(\lambda)\right)^{2} \cdot r_{g}(\lambda)}{1-r_{g}(\lambda) r_{i}\left(\sum_{j} a_{j} t_{j}(\lambda)^{2}\right)} \cdot \frac{1-R_{12}\left(\theta_{1}^{\text {out }}\right)}{n_{2}^{2}}
$$

Most handheld instruments measure the reflectance factor at a $\left(45^{\circ}: 0^{\circ}\right)$ geometry. At the exit angle of $0^{\circ}$ and with $n_{2}=1.5$, the term $\left(1-R_{12}\left(\theta_{1}^{\text {out }}\right)\right) / n_{2}^{2}$ is numerically close to $\left(1-r_{i}\right)$, see Table 3 in Section 6. Therefore, even for these handheld instruments, Eq. (37) with $K=0$ is often used instead of Eq. (42). Note that for a measuring geometry composed of a narrow solid angle sensor capturing reflected light at an angle $\theta$, the radiance factor defined as the ratio of captured radiances is the same as the reflectance factor defined as the ratio of captured irradiances. 


\subsection{Generalization of the Clapper-Yule model in order to account for low scattering within the paper bulk}

The classical Clapper-Yule assumes that the probability of a light ray to hit from below a given colorant surface is equal to the relative size of that colorant surface. There is no memory of where the corresponding light ray came from. This means that the average lateral propagation of light is large, i.e at least as large as half the screen element period. However, at screen frequencies below 120 lpi, this condition is not fulfilled and the classical Clapper-Yule model shows poor results. Rogers [36] generalized the classical Clapper-Yule model by assuming that within each light reflection cycle, the lateral propagation of light is modeled by a point spread function. A diffusion probability matrix incorporates the probabilities that photons enter a colorant $i$ and exit from colorant $j$. With these probabilities, the halftone reflectance can be calculated. This provides a model capable of accounting also for middle and low screen frequencies.

A somehow simpler approach was proposed by Hersch et al. [37] which consists in performing a weighted average between the classical Clapper-Yule model and the Saunderson corrected Neugebauer model. Since the Neugebauer model assumes no lateral light scattering at all and the Clapper-Yule assume total lateral light scattering, a weighted average between the two models enables accounting for halftones having middle and low screen frequencies. From equation (32) we have for the reflectance of a solid ink layer of transmittance $t(\lambda)$ printed on a paper of reflectance $r_{g}(\lambda)$

$$
R_{\text {solid }}(\lambda)=\frac{\left(1-r_{s}\right) t(\lambda)^{2} \cdot r_{g}(\lambda) \cdot\left(1-r_{i}\right)}{1-r_{i} \cdot r_{g}(\lambda) \cdot t(\lambda)^{2}}
$$

where $r_{s}=R_{12}\left(\theta_{1}^{i n}\right)$ is the specular reflectance that is discarded by the measurement instrument, i.e. $K=0$.

Equation (43) can also express the reflectance of a Neugebauer primary whose colorant transmittance is $t_{j}(\lambda)$. According to the Neugebauer model, the reflectance of a halftone is a weighted average of the colorant reflectances, with the weights given by the colorant surface coverages $a_{j}$

$$
R(\lambda)=\left(1-r_{S}\right) \cdot\left(1-r_{i}\right) \cdot r_{g}(\lambda) \cdot \sum_{j} \frac{a_{j} \cdot t_{j}(\lambda)^{2}}{1-r_{i} \cdot r_{g}(\lambda) \cdot t_{j}(\lambda)^{2}}
$$


The proposed "low-scattering" extension of the Clapper-Yule model is a weighted average between the Saunderson corrected Neugebauer model described by Eq. (44) and the ClapperYule model described by Eq. (37):

$$
R(\lambda)=K r_{S}+\left(1-r_{S}\right) r_{g}(\lambda)\left(1-r_{i}\right)\left[b\left[\sum_{j} \frac{a_{j} \cdot t_{j}(\lambda)^{2}}{1-r_{i} \cdot r_{g}(\lambda) \cdot t_{j}(\lambda)^{2}}\right]+(1-b) \frac{\left(\sum_{j} a_{j} \cdot t_{j}(\lambda)\right)^{2}}{1-r_{g}(\lambda) \cdot r_{i} \cdot \sum_{j} a_{j} \cdot t_{j}(\lambda)^{2}}\right]
$$

Interpolation coefficient $b$ between 0 and 1 , indicates how close the model is to the Neugebauer model and coefficient $(1-b)$ indicates how close it is to the Clapper-Yule model. Optimal values for coefficient $b$ can be learned by performing predictions on a given number of halftone patches of known reflectances.

\subsection{Reflectance of light from a solid colored translucent layer on a white diffusing substrate (Williams and Clapper model)}

When light crosses an ink layer, its attenuation according to Beer's law depends on its path length, and therefore on its orientation in respect to the surface normal. This orientation dependent attenuation was ignored by Clapper and Yule who assumed that the error is small, particularly when the ink penetrates the paper and scatters light. However, an ink layer on top of a coated paper remains non-scattering and the orientation dependent attenuation may become significant. Williams and Clapper took the orientation dependent attenuation into account for the modelization of full tone photographs at the $45^{\circ}: 0^{\circ}$ geometry [22]. The same approach was later extended to any incident and exit angle [38] as well as to halftone prints [39].

Calculation of the radiance factor with a $\left(\theta_{1}^{\text {in }}: \theta_{1}^{\text {out }}\right)$ measurement geometry

Let us calculate the radiance factor when a solid colored layer on white diffusing substrate is illuminated at a given incident angle $\theta_{1}^{\text {in }}$ and the reflected radiance is captured at a given exit angle $\theta_{1}^{\text {out }}$

The print is illuminated by a collimated light beam of irradiance $E_{i}$ from the air-side of the interface with an incidence angle $\theta_{1}^{i n}$ (Figure 12). A fraction $R_{12}\left(\theta_{1}^{i n}\right)$ of the incident light is 
reflected into air along the specular direction. Since we assume that the detector is not placed in the specular direction, this external specular reflection is ignored.

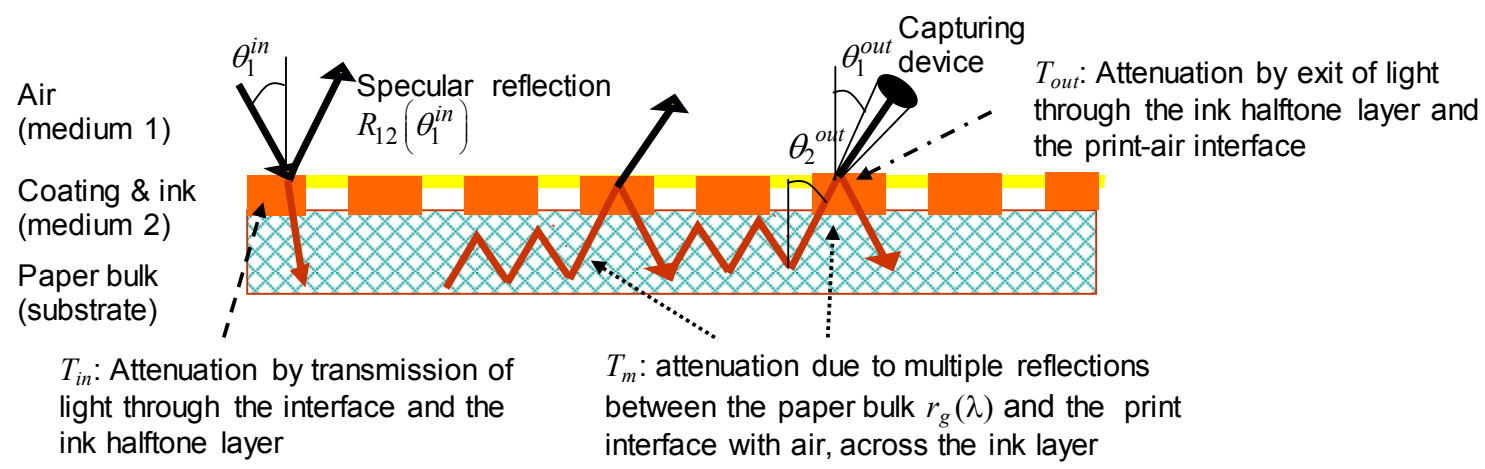

Figure 12: Path of a collimated beam of light from the source to the detector, with multiple internal reflections between the substrate and the colored layer-air interface.

The incident irradiance $E_{i}$ is directed towards the print at angle $\theta_{1}^{i n}$ and traverses the colored layer at angle $\theta_{2}^{i n}$, related to angle $\theta_{1}^{\text {in }}$ by Snell's law [hdi062, Eq. (30)]. The attenuation of incoming light at the interface is $T_{12}\left(\theta_{1}^{i n}\right)=1-R_{12}\left(\theta_{1}^{i n}\right)=1-R_{21}\left(\theta_{2}^{i n}\right)$ and the attenuation of light through the colored layer of length $1 / \cos \theta_{2}^{\text {in }}$ is, according to Beer's law, $t(\lambda)^{1 / \cos \theta_{2}^{\text {in }}}$. Before reaching the substrate for the first time, the incoming light beam is therefore attenuated by

$$
T_{\text {in }}(\lambda)=\left(1-R_{12}\left(\theta_{1}^{i n}\right)\right) t(\lambda)^{1 / \cos \theta_{2}^{i n}}=T_{12}\left(\theta_{1}^{i n}\right) t(\lambda)^{1 / \cos \theta_{2}^{i n}}
$$

The diffusing substrate reflects over the whole hemisphere a ratio $r_{g}(\lambda)$ of irradiance $T_{i n}(\lambda) E_{i}(\lambda)$ incident on it. This diffuse irradiance $E_{0}(\lambda)=r_{g}(\lambda) T_{i n}(\lambda) E_{i}(\lambda)$ is emitted from the substrate towards the colored layer, traverses the colored layer, is partially reflected at the colored layerair interface, traverses again the colored layer and reaches the substrate and is reflected from it for the second time. The attenuation of this single reflection cycle comprises the attenuation of traversing twice the colored layer, the Fresnel reflection at the colored layer-air interface and the diffusion-reflection by the substrate.

We can group the attenuation by the colored layer interface and the attenuation at the layer-air interface. For a given incident angle $\theta$ from below the layer-air interface, the attenuation is 
$R_{21}(\theta) t(\lambda)^{2 / \cos \theta}$. Since we must integrate over all possible incident angles, we obtain in a similar manner as when deriving Eq. (22) the attenuation $r_{i}(t, \lambda)$ of the diffuse incident irradiance

$$
r_{i}(t, \lambda)=\int_{0}^{\pi / 2} t(\lambda)^{2 / \cos \theta} R_{21}(\theta) \sin 2 \theta d \theta
$$

After one full reflection cycle, we obtain the attenuated irradiance $E_{1}(\lambda)=r_{i}(t, \lambda) r_{g}(\lambda) E_{0}(\lambda)$. At the $k^{\text {th }}$ full reflection cycle, we have the attenuated irradiance $E_{k}(\lambda)=\left[r_{i}(t, \lambda) r_{g}(\lambda)\right]^{k} E_{0}(\lambda)$. By summing all these attenuated irradiance components, we obtain the total irradiance $E_{S}(\lambda)$ emerging from the substrate, but still beneath the print-air interface

$$
E_{S}(\lambda)=\left(\sum_{k=0}^{\infty}\left(r_{g}(\lambda) r_{i}(t, \lambda)\right)^{k}\right) E_{0}(\lambda)=\left(\sum_{k=0}^{\infty}\left(r_{g}(\lambda) r_{i}(t, \lambda)\right)^{k}\right) r_{g}(\lambda) \cdot T_{i n}(\lambda) \cdot E_{i}(\lambda)
$$

which is a geometric series and can be expressed by

$$
E_{S}(\lambda)=\frac{1}{1-r_{g}(\lambda) r_{i}(t, \lambda)} E_{0}(\lambda)=\frac{r_{g}(\lambda)}{1-r_{g}(\lambda) r_{i}(t, \lambda)} T_{i n}(\lambda) \cdot E_{i}(\lambda)
$$

Therefore, the internal reflectance of the print comprising the attenuation of the colored layer, the Fresnel reflections at the interface and the intrinsic reflectance of the substrate is

$$
R_{m}(\lambda)=\frac{E_{S}(\lambda)}{E_{\text {in }}(\lambda) T_{\text {in }}(\lambda)}=\frac{r_{g}(\lambda)}{1-r_{g}(\lambda) r_{i}(t, \lambda)}
$$

Let us now compute the exit attenuation, which depends on the capturing device. Let us first assume a capturing device at exit orientation $\theta_{1}^{\text {out }}$. This device will capture light emitted from the substrate and traveling within the colored layer at angle $\theta_{2}^{\text {out }}$ which depends on $\theta_{1}^{\text {out }}$ by Snell's law (hdi062, Eq. (30)). Since the substrate is Lambertian, the radiance at any orientation and also at orientation $\theta_{2}^{\text {out }}$ is $E_{S}(\lambda) / \pi$. This radiance reaches the layer-air interface with an attenuation factor $t(\lambda)^{1 / \cos \theta_{2}^{\text {out }}}$ due to the transmittance of the colored layer. The radiance $L_{S}(\lambda)$ incident onto the layer-air interface is therefore

$$
L_{S}(\lambda)=\frac{E_{S}(\lambda)}{\pi}=t(\lambda)^{1 / \cos \theta_{2}^{\text {out }}} \frac{1}{\pi} \cdot \frac{r_{g}(\lambda)}{1-r_{g}(\lambda) r_{i}(t, \lambda)} T_{\text {in }}(\lambda) \cdot E_{i}(\lambda)
$$

According to Eq. (44) of hdi062, the radiance transmitted by an interface is the Fresnel transmittance of the interface $T_{21}\left(\theta_{2}^{\text {out }}\right)=T_{12}\left(\theta_{1}^{\text {out }}\right)=1-R_{21}\left(\theta_{2}^{\text {out }}\right)$ multiplied by its relative index of refraction, here $\left(n_{1} / n_{2}\right)^{2}=\left(1 / n_{2}\right)^{2}$. The exit attenuation is therefore 


$$
T_{\text {out }}(\lambda)=t(\lambda)^{1 / \cos \theta_{2}^{\text {out }}} T_{21}\left(\theta_{2}^{\text {out }}\right)\left(1 / n_{2}\right)^{2}
$$

We obtain for the radiance at exit orientation $\theta_{1}^{\text {out }}$

$$
L_{1}\left(\theta_{1}^{\text {out }}\right)=T_{\text {out }} L_{S}=T_{\text {out }} \frac{1}{\pi} R_{m} T_{\text {in }} E_{i}=\frac{T_{21}\left(\theta_{2}^{\text {out }}\right)}{\left(n_{2}\right)^{2}} t^{1 / \cos \theta_{2}^{\text {out }}} \frac{1}{\pi} \cdot \frac{r_{g}(\lambda)}{1-r_{g}(\lambda) r_{i}(t, \lambda)} T_{\text {in }}(\lambda) \cdot E_{i}(\lambda)
$$

The radiance factor expresses the attenuation of the exiting radiance at the selected angle in respect to the radiance reflected by a perfect white diffuse reflector. The radiance reflected by a perfect white diffuse reflector is $L_{r e f}(\lambda)=E_{i}(\lambda) / \pi$. We obtain the radiance factor $R_{f a c}(\lambda)$ of a colored layer on a diffusing substrate, illuminated at angle $\theta_{1}^{\text {in }}$ and measured at angle $\theta_{1}^{\text {out }}$

$$
\begin{aligned}
R_{\text {fac }}\left(\theta_{1}^{\text {out }}, \lambda\right) & =\frac{L_{1}\left(\theta_{1}^{\text {out }}\right)}{E_{i} / \pi}=T_{\text {out }} R_{m} T_{\text {in }} \\
& =\left(\frac{1-R_{21}\left(\theta_{2}^{\text {out }}\right)}{\left(n_{2}\right)^{2}} t(\lambda)^{1 / \cos \theta_{2}^{\text {out }}}\right) \frac{r_{g}(\lambda)}{1-r_{g}(\lambda) r_{i}(t, \lambda)}\left(\left(1-R_{12}\left(\theta_{1}^{\text {in }}\right)\right) t(\lambda)^{1 / \cos \theta_{2}^{\text {in }}}\right)
\end{aligned}
$$

This expression has been presented in a similar form by Shore and Spoonhower [38]. It reproduces the equation of Williams and Clapper [22] for the special case of $n_{2}=1.53, \theta_{1}^{\text {in }}=45^{\circ}$, and $\theta_{1}^{\text {out }}=0^{\circ}$.

Calculation of the reflectance by capturing the exiting irradiance with an integrated sphere, i.e. with a $\left(\theta_{1}^{\text {in }}: d\right)$ measurement geometry.

In the case that we measure the exiting irradiance $E_{1}$ with an integrated sphere, we obtain the exiting irradiance by integrating the exiting radiance $L_{1}\left(\theta_{1}^{\text {out }}\right)$ over the whole hemisphere

$$
E=\int_{0}^{2 \pi} \int_{0}^{\pi / 2} L_{1}(\theta) \cos \theta \sin \theta d \theta d \phi
$$

By inserting (53) into (55) we obtain

$$
E_{\text {out }}(\lambda)=\frac{r_{g}(\lambda)}{1-r_{g}(\lambda) r_{i}(t, \lambda)} T_{\text {in }}(\lambda) \cdot E_{i}(\lambda) \cdot 2 \int_{0}^{\pi / 2} \frac{T_{21}\left(\theta_{2}^{\text {out }}\right)}{\left(n_{2}\right)^{2}} t(\lambda)^{1 / \cos \theta_{2}^{\text {out }}} \cos \theta_{1}^{\text {out }} \sin \theta_{1}^{\text {out }} d \theta_{1}^{\text {out }}
$$

and with Eq. (42) of Chapter hdi062, $\cos \theta_{1}^{\text {out }} \sin \theta_{1}^{\text {out }} d \theta_{1}^{\text {out }}=n_{2}^{2} \cos \theta_{2}^{\text {out }} \sin \theta_{2}^{\text {out }} d \theta_{2}^{\text {out }}$

$$
E_{\text {out }}(\lambda)=\frac{r_{g}(\lambda)}{1-r_{g}(\lambda) r_{i}(t, \lambda)} T_{\text {in }}(\lambda) \cdot E_{i}(\lambda) \cdot 2 \int_{0}^{\pi / 2} T_{21}\left(\theta_{2}^{\text {out }}\right) t(\lambda)^{1 / \cos \theta_{2}^{\text {out }}} \cos \theta_{2}^{\text {out }} \sin \theta_{2}^{\text {out }} d \theta_{2}^{\text {out }}
$$

and by grouping the exit attenuation into the exit term $T_{\text {out }}$ 


$$
T_{\text {out }}(\lambda)=2 \int_{0}^{\pi / 2} T_{21}\left(\theta_{2}^{\text {out }}\right) t(\lambda)^{1 / \cos \theta_{2}^{\text {out }}} \cos \theta_{2}^{\text {out }} \sin \theta_{2}^{\text {out }} d \theta_{2}^{\text {out }}=\int_{0}^{\pi / 2} T_{21}\left(\theta_{2}^{\text {out }}\right) t(\lambda)^{1 / \cos \theta_{2}^{\text {out }}} \sin 2 \theta_{2}^{\text {out }} d \theta_{2}^{\text {out }}
$$

we obtain

$$
E_{\text {out }}(\lambda)=T_{\text {out }} R_{m} T_{\text {in }} \cdot E_{i}=T_{\text {out }}(\lambda) \frac{r_{g}(\lambda)}{1-r_{g}(\lambda) r_{i}(t, \lambda)} T_{\text {in }}(\lambda) \cdot E_{i}(\lambda)
$$

The reflectance $R$, measured with an integrated sphere, is therefore

$$
\begin{aligned}
R(\lambda) & =E_{\text {out }} / E_{i}=T_{\text {out }} R_{m} T_{\text {in }}=T_{\text {out }}(\lambda) \frac{r_{g}(\lambda)}{1-r_{g}(\lambda) r_{i}(t, \lambda)} T_{\text {in }}(\lambda) \\
& =\left(\int_{0}^{\pi / 2}\left(1-R_{21}\left(\theta_{2}^{\text {out }}\right)\right) t(\lambda)^{1 / \cos \theta_{2}^{\text {out }}} \sin 2 \theta_{2}^{\text {out }} d \theta_{2}^{\text {out }}\right) \frac{r_{g}(\lambda)}{1-r_{g}(\lambda) r_{i}(t, \lambda)}\left(1-R_{12}\left(\theta_{1}^{\text {in }}\right) t(\lambda)^{1 / \cos \theta_{2}^{\text {in }}}\right)
\end{aligned}
$$

In practice, a measurement instrument measures a reflectance factor, i.e. the irradiance reflected by the colored layer on the diffuse substrate captured by the integrated sphere divided by the irradiance of a perfect Lambertian diffuser captured by the same integrated sphere. Since all incident irradiance is reflected into the integrated sphere, the reflectance factor and the reflectance are identical.

\section{Reflectance of light from a color halftone translucent layer on a white diffusing substrate (Williams-Clapper extended to color halftones)}

In case that the considered halftone screen frequency is high (e.g. 120 lpi or higher), we can assume that the lateral propagation of light due to scattering within the substrate and to multiple reflections between the substrate and the layer-air interface is important in respect to the screen period. In that case, light reflected by the substrate towards the color halftone layer has a probability to strike a given colorant (Neugebauer primary) equal to that colorant surface coverage. The location hit by a diffused and reflected light beam is independent of the location from which light entered the layer.

In the case of a high halftone screen frequency, we can simply extend the reflectance from a solid translucent layer to the reflectance of a color halftone translucent layer by replacing in Eqs. (46), (50), and (52) the single ink solid layer by a layer composed of multiple colorants whose surface coverages $a_{j}$ are known. The terms $T_{i n}$ and $R_{m}$ common to both equations become

$$
T_{\text {in }}(\lambda)=\left(1-R_{12}\left(\theta_{1}^{i n}\right)\right) \sum_{j} a_{j} t(\lambda)_{j}^{1 / \cos \theta_{2}^{i n}}
$$




$$
R_{m}(\lambda)=\frac{r_{g}(\lambda)}{1-r_{g}(\lambda) \sum_{j} a_{j} r_{i}\left(t_{j}, \lambda\right)}
$$

where $r_{i}\left(t_{j}, \lambda\right)$ is calculated in Equation (47) for ink $j$ of normal transmittance $t_{j}(\lambda)$.

The exit term $T_{\text {out }}$ in Eq. (52) for the radiance factor becomes

$$
T_{\text {out }}(\lambda)=\frac{1-R_{21}\left(\theta_{2}^{\text {out }}\right)}{\left(n_{2}\right)^{2}} \sum_{j} a_{j} \cdot t(\lambda)_{j}^{1 / \cos \theta_{2}^{\text {out }}}
$$

and the exit term $T_{\text {out }}$ in Eq. (60) for the reflectance becomes

$$
T_{\text {out }}(\lambda)=\sum_{j}\left(a_{j} \cdot \int_{0}^{\pi / 2}\left(1-R_{21}\left(\theta_{2}^{\text {out }}\right)\right) t(\lambda)_{j}^{1 / \cos \theta_{2}^{\text {out }}} \sin 2 \theta_{2}^{\text {out }} d \theta_{2}^{\text {out }}\right)
$$

We obtain the following full equation for the radiance factor of a colored halftone illuminated at angle $\theta_{1}^{\text {in }}$ and measured at angle $\theta_{1}^{\text {out }}$

$$
\begin{aligned}
R_{\text {fac }}\left(\theta_{1}^{\text {out }}, \lambda\right) & =\frac{L_{1}\left(\theta_{1}^{\text {out }}\right)}{E_{i} / \pi}=T_{\text {out }} T_{m} T_{\text {in }} \\
& =\left(\frac{1-R_{21}\left(\theta_{2}^{\text {out }}\right)}{\left(n_{2}\right)^{2}} \sum_{j} a_{j} t(\lambda)_{j}^{1 / \cos \theta_{2}^{\text {out }}}\right) \frac{r_{g}(\lambda)}{1-r_{g}(\lambda) \sum a_{j} r_{i}\left(t_{j}, \lambda\right)}\left(\left(1-R_{12}\left(\theta_{1}^{\text {in }}\right)\right) \sum_{j} a_{j} t(\lambda)_{j}^{1 / \cos \theta_{2}^{\text {in }}}\right)
\end{aligned}
$$

and the following full equation for the Williams-Clapper reflectance of a colored halftone captured by an integrated sphere

$$
\begin{aligned}
R(\lambda)= & \sum_{j}\left(a_{j} \int_{0}^{\pi / 2}\left(1-R_{21}\left(\theta_{2}^{\text {out }}\right)\right) t(\lambda)_{j}^{1 / \cos \theta_{2}^{\text {out }}} \sin 2 \theta_{2}^{\text {out }} d \theta_{2}^{\text {out }}\right) \\
& \frac{r_{g}}{1-r_{g}(\lambda) \sum_{j} a_{j} r_{i}\left(t_{j}, \lambda\right)}\left(\left(1-R_{12}\left(\theta_{1}^{\text {in }}\right)\right) \sum_{j} a_{j} t(\lambda)_{j}^{1 / \cos \theta_{2}^{\text {in }}}\right)
\end{aligned}
$$

The integrals in expressions (64) and (66) as well as the Fresnel reflectance $r_{i}\left(t_{j}, \lambda\right)$ at the colored interface may be computed as discrete sums by sampling the integrated terms in respect to angle $\theta$ with a small sampling step of for example $\Delta \theta=0.001 \mathrm{rad}$. It is also possible to replace the integrals by analytical expressions which offer an accurate approximation. 
Equations (67) and (68) are approximations of the internal reflectance $r_{i}\left(t_{j}, \lambda\right)$ and of the single colorant output transmittance

$$
\begin{gathered}
r_{i}\left(t_{j}, \lambda\right)=\int_{\theta=0}^{\pi / 2} R_{21}(\theta) t(\lambda)_{j}^{2 / \cos \theta} \sin 2 \theta d \theta \simeq \frac{e^{t j(\lambda)^{\gamma}}-1}{e-1} r_{21} \\
\int_{0}^{\pi / 2}\left(1-R_{21}\left(\theta_{2}^{\text {out }}\right)\right) t(\lambda)_{j}^{1 / \cos \theta_{2}^{\text {out }}} \sin 2 \theta_{2}^{\text {out }} d \theta_{2}^{\text {out }}=\int_{\theta=0}^{\pi / 2} T_{21}(\theta) t(\lambda)^{1 / \cos \theta} \sin 2 \theta d \theta \approx t(\lambda)^{\mu} t_{21}
\end{gathered}
$$

where $R_{21}(\theta)$ is the Fresnel reflection coefficient at the paper side of the print-air interface, $T_{21}(\theta)$ is the paper-to-air Fresnel transmission coefficient, $r_{21}=r_{i}$ is the average Fresnel reflection of Lambertian light at the print-air interface, $t_{21}=1-r_{i}$ is the corresponding average Fresnel transmission across the paper-air interface and $\gamma$ and $\mu$ are coefficients depending on the refractive index of the paper. For a typical paper refractive index of 1.5 , we have $r_{i}=0.596$, $t_{21}=0.404, \gamma=2.945$ and $\mu=1.134[29]$.

\section{INK SPREADING MODELS}

Since the beginning of the $20^{\text {th }}$ century, researchers observed and tried to model the dot gain phenomenon. In early work, optical dot gain due to propagation of light within the print and physical dot gain due to the spreading of ink could not be distinguished. Since modern physical prediction models have the capability of estimating the optical dot gain, we are now coming closer to the goal of separating optical and physical dot gain. However, methods for establishing a clear separation between the two dot gain phenomena are still a subject of research.

The Murray-Davis formula given in Eq. (10) was often used to calculate the so-called "dot gain", which incorporates both the optical and the physical dot gain. In the early fifties of the

$20^{\text {th }}$ century, scientists started establishing a relationship between amount of inks or surface coverages of ink dots and the resulting printed color [40], [11].

Yule and Colt [40] analyzed the superposition of one ink over two other inks. They observed an increased spreading of the overlapping ink and came up with a formula for calculating the effective surface coverage of an ink dot located on top of other ink dots:

$$
a^{\prime}=1-(1-a)^{(1+u)}
$$


where $a$ is the nominal surface coverage of the considered ink dot, $a^{\prime}$ is its effective surface coverage and $u$ is the overall surface coverage (i.e. the covered surface) of the two other underlying ink dots. This formula has the advantage that the effective surface coverage is never larger than 1. Figure 13 shows the dot gain curves for different values of the underlying total surface $u$.

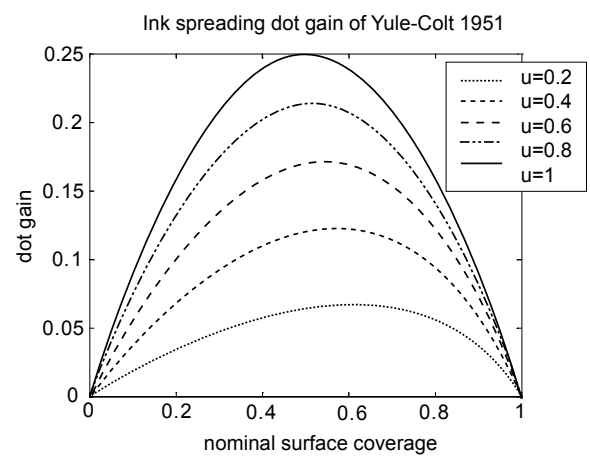

Figure 13: Dot gain $d_{g}=a^{\prime}-a$ due to ink spreading according to the Yule and Colt formula.

In 1983, Viggiano [41] proposed a different dot gain model relying on effective surface coverages in function of nominal surface coverages:

$$
a^{\prime}=a+2 \cdot v_{50 \%} \cdot \sqrt{a \cdot(1-a)}
$$

where $a$ is the nominal surface coverage of the considered ink dot, $a^{\prime}$ is its effective surface coverage and $v_{50 \%}$ is the amount of dot gain (defined as effective minus nominal surface coverage) at a nominal surface coverage of 0.5 . The dot gain $v_{50 \%}$ is to be estimated or determined from actual measurements of prints. This effective surface coverage function produces effective surface coverage values larger than 1 at nominal surface coverage above 0.9 , when significant dot gain is present (e.g. a dot gain of 0.2 at $50 \%$ nominal surface coverage).

In several works, e.g. [16], the curve mapping nominal surface coverages to effective surface coverages is formed by a parabola interpolating between the points $(0,0),\left(0.5,0.5+v_{50 \%}\right)$, and $(1,1)$. Value $v_{50 \%}$ expresses the dot gain at $50 \%$ nominal surface coverage. This parabola is described by the equation

$$
a^{\prime}=-4 v_{50 \%} a^{2}+4 v_{50 \%} a+a
$$

The corresponding dot gain curve (Figure 14) is

$$
d_{g}=a^{\prime}-a=-4 v_{50 \%} a^{2}+4 v_{50 \%} a
$$



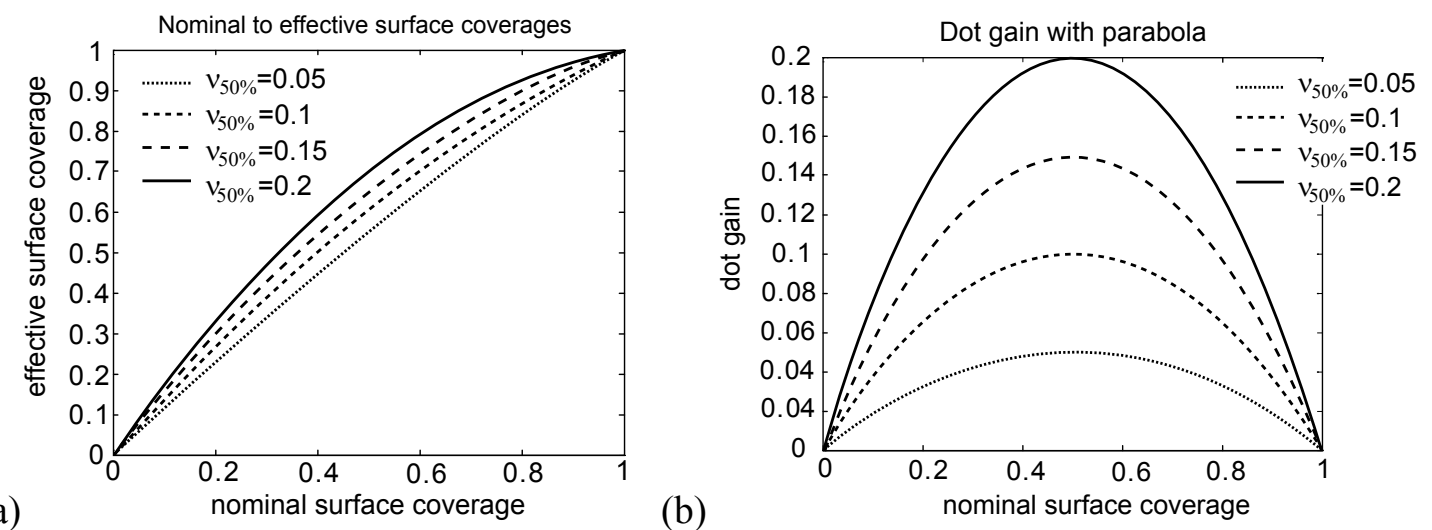

Figure 14: (a) Nominal to effective surface coverages given by the parabola Eq. (71) and (b) corresponding dot gain curve expressed by the parabola Eq. (72).

It is easy to show that if the dot gain $v_{50 \%}$ is between -0.25 and +0.25 at $50 \%$ nominal surface coverage, the corresponding nominal to effective surface coverage curve will not overshoot, i.e. all effective surface coverage values remain in the allowed range between 0 and 1.

Note that it is not necessary to have a smooth curve expressing the mapping between nominal and effective surface coverages of an ink halftone. Once several effective surface coverages are known, e.g. effective surface coverages $a_{0.25}^{\prime}, a_{0.5}^{\prime}, a_{0.75}^{\prime}$ at respective nominal surface coverages $25 \%, 50 \%$ and $75 \%$, one may establish as mapping between nominal and effective surface coverages the polyline connecting successive points, e.g. line $\left[(0,0),\left(0.25, a_{0.25}^{\prime}\right)\right.$, line $\left[\left(0.25, a_{0.25}^{\prime}\right),\left(0.5, a_{0.5}^{\prime}\right)\right]$, and line $\left[\left(0.5, a_{0.5}^{\prime}\right),\left(0.75, a_{0.75}^{\prime}\right),(1,1)\right]$.

Most approaches for capturing the physical dot gain of halftones of a given ink rely on a spectral model predicting the reflectance of a constant color halftone patch. By minimizing the sum of square differences between predicted reflectance and measured reflectance components, one may fit the corresponding effective dot surface coverage. As example, consider a halftone of ink $i$ printed on top of a full tone of ink $j$. The predicted reflectance $\hat{R}_{i / j}$ is obtained in this example with the YNSN model (Eq. (73)), and the unknown effective surface coverage $a_{i, j}{ }^{\prime}$ of halftone ink is fitted by minimizing the sum of square reflectance spectra component differences (Eq. (74))

$$
\begin{aligned}
& \hat{R}_{i / j}(q, \lambda)=\left(q \cdot R_{i / j}(\lambda)^{1 / n}+(1-q) \cdot R_{j}(\lambda)^{1 / n}\right)^{n} \\
& a_{i, j}^{\prime}=\underset{0 \leq q \leq 1}{\arg \min } \sum_{k}\left[R_{i / j}^{\text {meas }}\left(a_{i, j}, \lambda_{k}\right)-\hat{R}_{i / j}\left(q, \lambda_{k}\right)\right]^{2}
\end{aligned}
$$


This minimization can be performed either with an optimization function such as Matlab's fminsearch or fmincon functions, or by performing a least square calculation. In classical spectral prediction models, there is generally one "tone reproduction" curve per contributing ink, i.e. one curve per ink mapping nominal to effective ink halftone surface coverages [14].

However, tone reproduction curves are not necessarily the same when considering an ink halftone printed on paper or an ink halftone printed on another ink. This is the reason why some researchers advocate deducing the tone reproduction curves from multi-ink halftones [42].

Iino and Berns [43] proposed a further method accounting for physical dot gain or loss (e.g. due to trapping) of one halftone in superposition with other ink halftones. They assumed that the dot gain of an ink halftone printed in superposition with another ink halftone (e.g. $\left.d_{g}(y / m)\right)$ increases or decreases by a given surface coverage dependent factor $q_{y / m}(m)$ of the underlying ink, compared with the dot gain $d_{g}(y)$ of the ink halftone printed alone on paper.

$$
\begin{array}{ll}
d_{g}(y / m)=d_{g}(y) \cdot q_{y / m}(m) & \text { for yellow printed on a magenta halftone } \\
d_{g}(y / c)=d_{g}(y) \cdot q_{y / c}(c) & \text { for yellow printed on a cyan halftone }
\end{array}
$$

They also assume that a halftone superposed with two ink halftones further increases or respectively decreases its size according to a multiplicative combination of the contributing factors.

$$
d_{g}(y / m c)=d_{g}(y) \cdot q_{y / m}(m) \cdot q_{y / c}(c)
$$

This manner of expressing dot gain may work in well behaved printing systems such as offset (Figure 15a). However, in many printing systems, these equations are not applicable since a halftone superposed with two inks behaves differently compared with the same halftone printed on each of the two inks separately (Figure 15b).

Let us now present a state of the art ink spreading model accounting for ink spreading in all superposition conditions [44], [37], [45]. Ink spreading is present when an ink halftone is printed on top of paper, in superposition with another solid ink or in superposition with two or more solid inks. In a similar manner as the physical dot gain of a single ink halftone patch printed on paper, ink spreading tends to enlarge the effective surface of a printed dot and tends to lower the resulting reflection spectrum, i.e. it yields darker colors. However, there are cases where effective surface coverages of halftones superposed on other inks are not larger than the corresponding effective surface coverages of the same halftone printed alone on paper. Figure 15 (a) and (b) show examples of physical dot gain, defined as the effective surface coverage 
minus the nominal surface coverage, for a clustered dot ink halftone printed alone on paper and printed in superposition with one or two other solid inks.
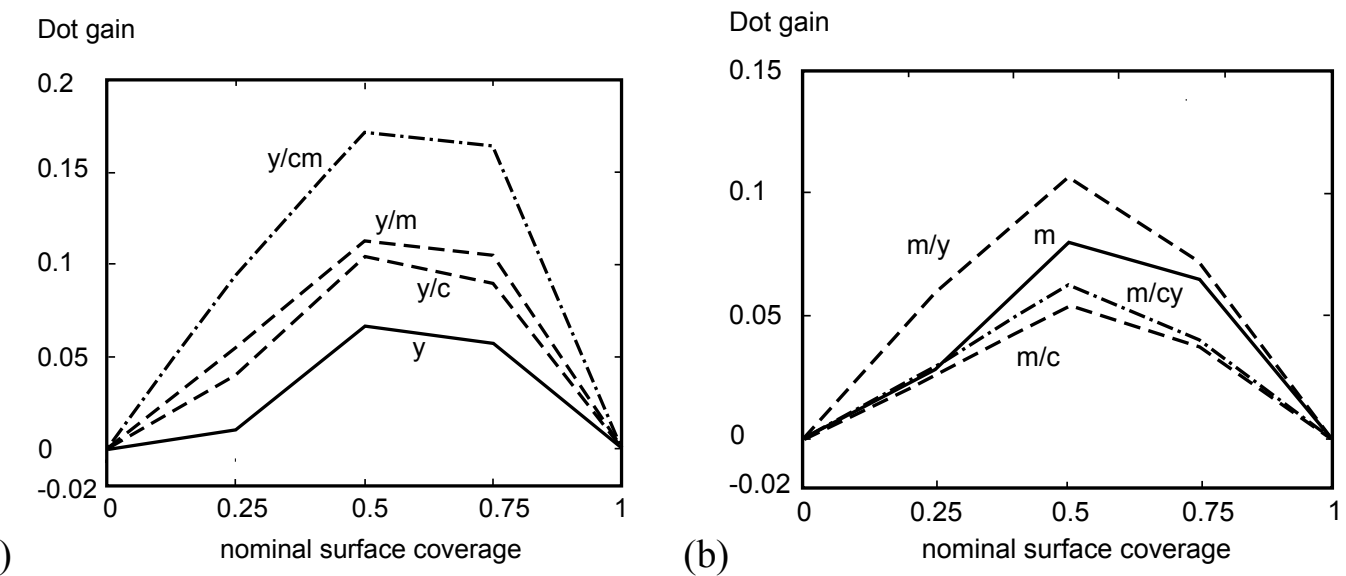

Figure 15: Dot gain as a function of nominal surface coverage, for ink halftones printed alone (solid line) and in superposition with the other solid inks (dotted lines), in the case of (a) an offset print at 150 lpi and (b) a thermal transfer print at 75 lpi.

Figure 15 (a) and (b) clearly show that the dot gain of an ink halftone is completely different when the ink halftone is printed on paper or when it is superposed with a second or third ink layer. In order to account for superposition dependent ink spreading, functions are created which map nominal to effective surface coverages of an ink halftone at each superposition condition. These functions define the ink spreading curves (e.g. Figure 14a).

There is one ink spreading curve for each ink halftone in each superposition condition. For example, a cyan halftone may be printed alone, $c$; superposed with solid magenta, $c / m$; with solid yellow, $c / y$; with solid black, $c / k$; with solid magenta and solid yellow, $c / m y$; with solid magenta and solid black, $c / m k$; with solid yellow and solid black, $c / y k$; and with solid magenta, yellow and black, c/myk. With a 4 ink CMYK printer, there are 8 different ink spreading curves for each ink, yielding a total of 32 ink spreading curves. However, since any halftone superposed with solid black yields a reflection spectrum very close to the reflection spectrum of solid black, ink spreading curves where one ink halftone is superposed with solid black are discarded [45]. In the case of cyan, magenta, yellow and black inks, Table 2 lists all the considered ink spreading curves for CMYK prints. In the case of CMY prints, only the first three columns need to be considered. 
Table 2. List of the considered ink spreading curve indices for CMYK ink prints.

\begin{tabular}{|c|c|c|c|c|}
\hline Cyan & Magenta & Yellow & \multicolumn{2}{|c|}{ Black } \\
\hline$c$ & $m$ & $y$ & $k$ & $k / y$ \\
\hline$c / m$ & $m / c$ & $y / c$ & $k / c$ & $k / c y$ \\
\hline$c / y$ & $m / y$ & $y / m$ & $k / m$ & $k / m y$ \\
\hline$c / m y$ & $m / c y$ & $y / c m$ & $k / c m$ & $k / c m y$ \\
\hline
\end{tabular}

Each ink spreading curve is calibrated using one or more so-called calibration samples, e.g. a halftone at $50 \%$ nominal surface coverage or three halftones at $25 \%, 50 \%$ and $75 \%$ nominal surface coverages (Figure 20). Calibration samples are obtained by asking the printer to print the halftones at their nominal surface coverages. Each sample determines one point of the ink spreading curve. Their effective surface coverages are fitted by minimizing a difference metric such as the sum of square differences between measured and predicted spectral reflectance components (see Eq. (74)). The ink spreading curves are obtained by linear interpolation between the fitted effective surface coverages. They may also be created by laying out a parabola through a single effective surface coverage, as shown in Eq. (71).

In order to avoid the ambiguity between chromatic black and pure black [48], the spectral measurements span both the visible wavelength range $(380-730 \mathrm{~nm})$ and the near infrared (NIR) wavelength range $(730-850 \mathrm{~nm})$. The NIR wavelength range enables distinguishing a light absorbing pigment-based black ink from the superposition of CMY inks, which are generally dye-based and do not absorb light in the NIR wavelength range.

The number of constant color samples to print can be deduced from the number of ink halftone superpositions (see Table 2) and from the number of solid colorants. In case of 3 inks, there are $2^{3}=8$ solid colorants and 12 superposition conditions. With one ink spreading calibration sample per superposition condition, we obtain a total of 20 samples whose reflectances need to be measured. In the case of the 4 cmyk inks, there are $2^{4}=16$ solid colorants and 20 superposition conditions (Table 2). With one ink spreading calibration sample per superposition condition, we obtain a total of 36 samples whose reflectances need to be measured.

As shown in Figure 16a, in a real halftone, halftone dots of one ink are superposed with halftone dots of the other inks. The ink spreading curves are acquired by superposing single ink halftones on solid surfaces of the other inks. For a given multi-chromatic halftone, we need to calculate the effective surface coverage of each of its ink halftones. For this purpose, we 
assume that a given ink dot surface is decomposed into partial dot surfaces which are each one superposed with a specific combination of the other inks and the paper. These partial dot surfaces can be assimilated to halftone elements on top of a solid colorant. Then, the effective surface coverage of an ink halftone is calculated as a weighted average of the different ink spreading curves. The weights correspond to the relative sizes of these partial dot surfaces.

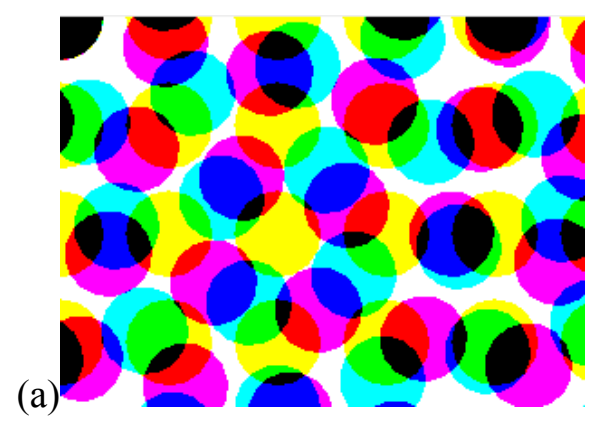

(b)

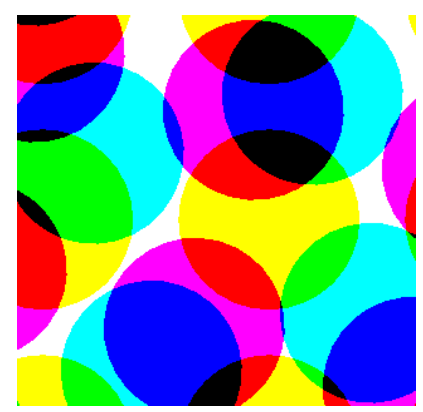

Figure 16: (a) Illustration of a three ink clustered dot color halftone with the cyan layer at orientation $15^{\circ}$, the magenta layer at orientation $75^{\circ}$ and the yellow layer at orientation $0^{\circ}$ and (b) enlarged part of the halftone showing the new colorants red, green, blue in regions where two of the cyan, magenta and yellow inks overlap and black where the three inks overlap.

When looking at the enlarged cyan halftone dot in Figure 16b, we see that this halftone dot is decomposed into partial dot surfaces superposed with the white colorant (pure cyan), the magenta colorant (pure blue), the yellow colorant (pure green) and the red colorant (chromatic black). In order to weight the ink spreading curves of cyan on white $f_{c}$, cyan on magenta $f_{c / m}$, cyan on yellow $f_{c / y}$, and cyan on red $f_{c / m y}$, we need to estimate the surface coverages of the white, magenta, yellow, and red colorants beneath the cyan dot. In case of halftone layers laid out independently one from another, the Demichel equations allow deducing surface coverages of colorants from surface coverages of inks. Let us lift the cyan layer from the halftone shown in Figure 16. Superposed with the cyan layer are the colorants white, magenta, yellow and red. Their respective surface coverages are according to the Demichel equations respectively $a_{w}$ $=(1-m)(1-y), a_{m}=m(1-y), a_{y}=y(1-m)$, and $a_{r}=m y$. These surface coverages are used as weighting factors for the ink spreading curves $f_{c}, f_{c / m}, f_{c / y}$, and $f_{c / m y}$. We therefore obtain for the effective surface coverage of the cyan halftone

$$
c^{\prime}=\left(1-m^{\prime}\right)\left(1-y^{\prime}\right) f_{c}(c)+m^{\prime}\left(1-y^{\prime}\right) f_{c / m}(c)+\left(1-m^{\prime}\right) y^{\prime} f_{c / y}(c)+m^{\prime} y^{\prime} f_{c / m y}(c)
$$

In the case of 3 inks, we obtain the effective surface coverages of the cyan, magenta and yellow inks by performing the weighted average of the ink spreading curves as follows: 


$$
\begin{array}{cccc}
c^{\prime} & =\left(1-m^{\prime}\right) & \left(1-y^{\prime}\right) & f_{c}(c) \\
& +m^{\prime} & \left(1-y^{\prime}\right) & f_{c / m}(c) \\
& +\left(1-m^{\prime}\right) & y^{\prime} & f_{c / y}(c) \\
& +m^{\prime} & y^{\prime} & f_{c / m y}(c) \\
m^{\prime} & =\left(1-c^{\prime}\right) & \left(1-y^{\prime}\right) & f_{m}(m) \\
& +c^{\prime} & \left(1-y^{\prime}\right) & f_{m / c}(m) \\
& +\left(1-c^{\prime}\right) & y^{\prime} & f_{m / y}(m) \\
& +c^{\prime} & y^{\prime} & f_{m / c y}(m) \\
y^{\prime} & =\left(1-c^{\prime}\right) & \left(1-m^{\prime}\right) & f_{y}(y) \\
& +c^{\prime} & \left(1-m^{\prime}\right) & f_{y / c}(y) \\
& +\left(1-c^{\prime}\right) & m^{\prime} & f_{y / m}(y) \\
& +c^{\prime} & m^{\prime} & f_{y / c m}(y)
\end{array}
$$

In case of the 4 CMYK inks, we have an additional equation for the black ink:

$$
\begin{aligned}
& k^{\prime}=\left(1-c^{\prime}\right)\left(1-m^{\prime}\right)\left(1-y^{\prime}\right) f_{k}(k) \\
& +c^{\prime}\left(1-m^{\prime}\right)\left(1-y^{\prime}\right) f_{k / c}(k) \\
& +\left(1-c^{\prime}\right) \quad m^{\prime} \quad\left(1-y^{\prime}\right) f_{k / m}(k) \\
& +c^{\prime} \quad m^{\prime} \quad\left(1-y^{\prime}\right) f_{k / c m}(k) \\
& +\left(1-c^{\prime}\right)\left(1-m^{\prime}\right) \quad y^{\prime} \quad f_{k / y}(k) \\
& +c^{\prime}\left(1-m^{\prime}\right) \quad y^{\prime} \quad f_{k / c y}(k) \\
& +\left(1-c^{\prime}\right) \quad m^{\prime} \quad y^{\prime} \quad f_{k / m y}(k) \\
& +\quad c^{\prime} \quad m^{\prime} \quad y^{\prime} \quad f_{k / c m y}(k)
\end{aligned}
$$

Since other inks printed on the black ink yield black, it makes no sense to account for the dot gain of an ink superposed with black. Therefore, the corresponding superposition conditions do not appear in Eqs. (75). But the superposition of a black ink halftone with another ink yields a clearly identifiable physical dot gain. Since there are 8 superposition conditions of a black halftone, Eq. (76) has 8 terms.

We solve Eqs. (75) and (76) iteratively, starting by assigning at the right-hand part of the equations the nominal ink halftone surface coverages $(c, m, y, k)$ to the effective ink halftone surface coverages $\left(c^{\prime}, m^{\prime}, y^{\prime}, k^{\prime}\right)$. After one iteration, we obtain new values for $\left(c^{\prime}, m^{\prime}, y^{\prime}, k^{\prime}\right)$ which are used for the next iteration on the right part of the equations. Four to five iterations ensure sufficient convergence to determine the effective ink halftone surface coverages. 
The complete Yule-Nielsen modified spectral prediction model, with the ink spreading extension is shown in Figure 17. The input to the spectral prediction model is given by the desired amounts of inks, or equivalently the nominal surface coverages $c, m, y, k$ to be printed. Thanks to the ink spreading functions $f_{i j k k}$, one may calculate the effective ink surface coverages $c^{\prime}, m^{\prime}, y^{\prime}, k^{\prime}$. From these effective surface coverages, one obtains with the Demichel equations (4) the effective surface coverages of the colorants (Neugebauer primaries) which are fed into the selected prediction model, here the Yule-Nielsen modified spectral Neugebauer model. As output, one obtains the predicted reflectance spectrum.

The ink spreading models presented so far either assume uniform thickness halftone dots, or assume that from a macroscopic point of view, the halftone dot profile thickness variations are accounted for by fitting the dots effective surface coverages [46]. There are however ink spreading models that explicitly provide a model of the printed pixel dot thickness profiles. These pixel dot thickness profiles depend on the presence or absence of neighboring pixel dots. One of these models adapted to ink-jet printing, is proposed by Emmel and Hersch [47].

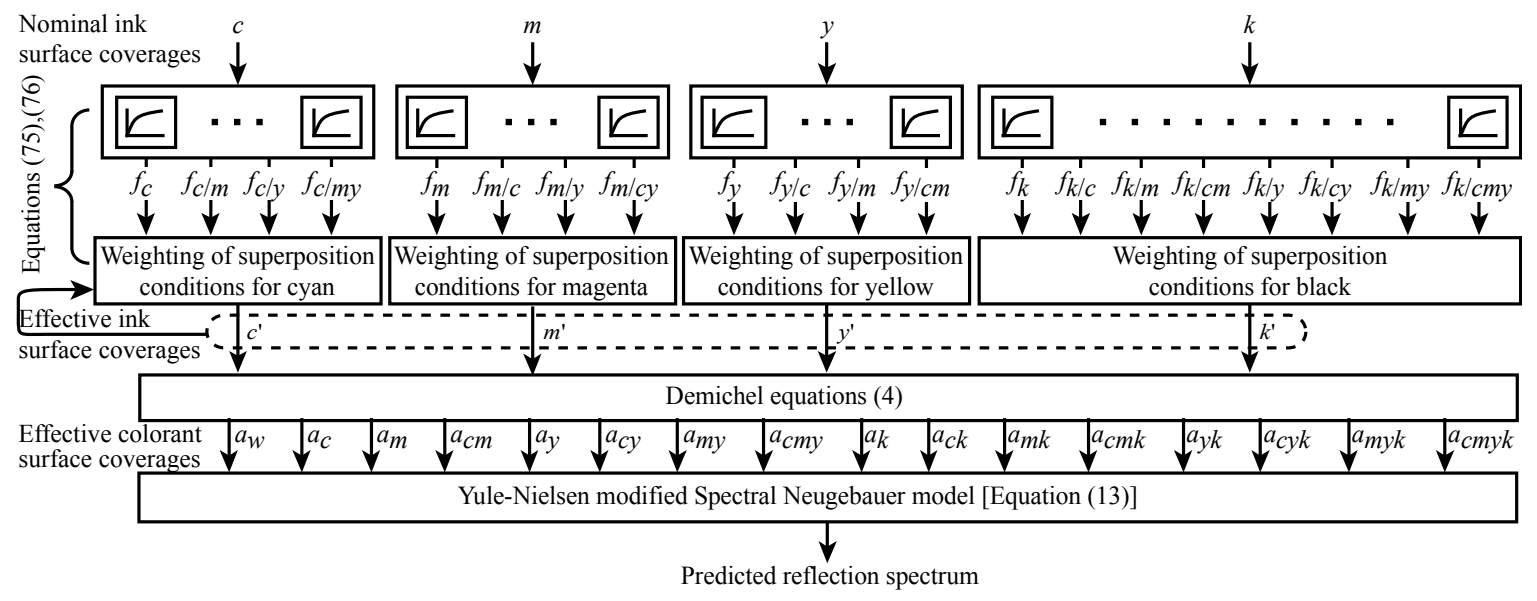

Figure 17: The ink spreading enhanced Yule-Nielsen modified Spectral Neugebauer model with nominal ink surface coverages $c, m, y$, and $k$; ink spreading curves $f_{i j k}$ of ink $i$ superposed with solid inks $j$ and $k$; effective ink surface coverages $c^{\prime}, m^{\prime}, y^{\prime}$, and $k^{\prime}$; and effective colorant surface coverages $a_{w}$ to $a_{c m y k}$.

\section{CALIBRATION OF THE MODELS}

When considering a given printing setup, one is a priori unable to estimate how much light at the different wavelengths is reflected by the paper or absorbed by the inks and how much the 
ink dots spread on the paper surface, until specific spectral measurements are performed. The spectral properties of the paper and inks are represented in each model by spectral reflectances or transmittances deduced from the spectral reflectance of full-tone colors. The growth of the ink dots, i.e. the dot gain, is assessed by establishing the correspondence between nominal and effective surface coverages for each ink halftone, thanks to nominal-to-effective surface coverage functions, also called ink spreading functions. The spectral reflectances, respectively transmittances, and the ink spreading functions are computed in a calibration procedure which ensures the ability of the model to account for the specific properties of the considered print.

In this section, we give an overview of the calibration of the Spectral Neugebauer (SN), YuleNielsen modified Spectral Neugebauer (YNSN), Clapper-Yule, Low Scattering Clapper-Yule (LSCY), and Williams-Clapper models for 3-ink halftones. We first explain how the spectral parameters of each model are obtained and then present two dot-gain assessment methods which are applicable to all models: the independent ink spreading (IIS) method and superposition-dependent ink spreading (SDIS) method. Since every calibrated prediction model is a combination of a base model and a dot-gain assessment method, both are specified in the model denomination, e.g. the "IIS-YNSN model". The calibration of the Cellular Yule-Nielsen modified Spectral Neugebauer (CYNSN) model is different and is presented separately. We also explain how predictions are performed from the calibrated models and finally discuss the necessity to perform a new calibration when parameters of the printing setup are modified.

\section{Spectral parameters}

Every prediction model incorporates a general equation expressing a function of spectral parameters which assess the absorbing and reflecting properties of the paper and inks. These spectral parameters are deduced from the spectral reflectances of the eight patches shown in row A in Figure 18, which correspond to solid layers of the eight colorants: the unprinted paper (white colorant), the paper printed with one solid ink layer (cyan, magenta and yellow colorants), with two solid ink layers (red, green and blue colorants) and with the three solid ink layers (chromatic black colorant). Let us denote their spectral reflectances as $R_{j}(\lambda), j=1, \ldots, 8$ and show how the spectral parameters of each model are obtained. 
Spectral Neugebauer (SN) and Yule-Nielsen enhanced Spectral Neugebauer (YNSN) models

The SN and YNSN equations are directly expressed in terms of the measured spectral reflectances $R_{j}(\lambda)$. They rely on equation (13)

$$
R(\lambda)=\left[\sum_{j} a_{j} R_{j}^{1 / n}(\lambda)\right]^{n}
$$

where the $n$ parameter is 1 in the SN model, and can be given an optimal value in the YNSN model using a set of patches of known spectral reflectances, for example the patches in rows $\mathrm{C}$ and $\mathrm{F}$ of Figure 18.

\section{Clapper-Yule and Low-Scattering Clapper-Yule models (LSCY)}

The Clapper-Yule model relies on Equation (78), which contains two types of spectral parameters: the spectral reflectance $r_{g}(\lambda)$ of the diffusing paper substrate, and the spectral transmittances $t_{j}(\lambda), j=1, \ldots, 8$ of the colorant layers

$$
R(\lambda)=K \cdot r_{s}+\frac{\tau_{\text {in }} \tau_{\text {out }}\left(\sum_{j} a_{j} t_{j}(\lambda)\right)^{2} r_{g}(\lambda)}{1-r_{i} r_{g}(\lambda)\left(\sum_{j} a_{j} t_{j}^{2}(\lambda)\right)}
$$

where $K, r_{s}, r_{i}, \tau_{\text {in }}$ and $\tau_{\text {out }}$ are constant parameters depending on the refractive index of the print and on the measuring geometry, but not on the wavelength. Note that according to formula (41), we have $\tau_{\text {in }}=1-R_{12}\left(\theta_{1}^{\text {in }}\right)$ and $\tau_{\text {out }}=1-R_{12}\left(\theta_{1}^{\text {out }}\right) / n_{2}^{2}$.

Table 3 gives numerical values for these terms when the refractive index is 1.5 , for three typical measuring geometries: the diffuse/eight degree geometry including or excluding the specular surface reflection (denoted respectively as di: $8^{\circ}$ and de: $8^{\circ}$ ) and the forty-five degree/zero degree geometry (denoted as $45^{\circ}: 0^{\circ}$ ). For more details on typical measuring geometries, see hdi062, Section 2.10.

The reflectance of the substrate, $r_{g}(\lambda)$, is deduced from the spectral reflectance of the unprinted support according to formula (27). The spectral colorant transmittances of the colorants are deduced from the spectral reflectances of the solid colorant patches according to formula (28). 
Table 3. Numerical values for the parameters of the Clapper-Yule model for three typical measuring geometries and a refractive index of 1.5 , with $R_{12}\left(0^{\circ}\right)=0.04$.

\begin{tabular}{llllll} 
Measuring geometry & $K$ & $r_{s}$ & $\tau_{\text {in }}$ & $\tau_{\text {out }}$ & $r_{i}$ \\
\hline di: $8^{\circ}$ & 1 & 0.09 & 0.91 & 0.43 & 0.596 \\
de: $8^{\circ}$ & 0 & 0.09 & 0.91 & 0.43 & 0.596 \\
$45^{\circ}: 0^{\circ}$ & 0 & 0.05 & 0.95 & 0.43 & 0.596 \\
\hline
\end{tabular}

Since the Low Scattering Clapper-Yule model is a linear combination of the Clapper-Yule and the Saunderson-Corrected Spectral Neugebauer models, its calibration consists in the calibration of both models and the optimization of the $b$ parameter from a set of patches of known spectral reflectance.

\section{Williams-Clapper model}

The Williams-Clapper model extended to halftones relies on equations (65) or (66) depending on whether the reflected light is captured by a radiance detector or, respectively, collected by an integrating sphere. Recall that no integral needs to be computed thanks to the approximating analytical functions proposed in Eqs. (67) and (68). The reflectance $r_{g}(\lambda)$ of the paper substrate is deduced from the spectral reflectance of the unprinted paper as in the Clapper-Yule model. The spectral transmittances $t_{i}(\lambda)$ of the colorants are deduced from the spectral reflectance of the solid colorant patches by solving numerically the equation, wavelength by wavelength, with the appropriate colorant surface coverages, i.e. for colorant $i, a_{j=i}=1$ and $a_{j \neq i}=0$.

\section{Ink spreading assessment methods}

Once the spectral parameters are obtained, we can start assessing the dot gain. The correspondence between the effective surface coverage of the inks and their nominal ones is represented by ink spreading functions as featured in Figure 19. Although each model (except the Spectral Neugebauer model) already accounts for optical dot gain, the effective ink surface coverages may also compensate for a possible under- or overestimation of optical dot gain. Two dot gain assessment methods are possible: the independent ink spreading (IIS) method where the spreading of each ink is assessed independently of the other inks, and the superposition-dependent ink spreading (SDIS) method where ink superposition configurations are taken into account. 
A
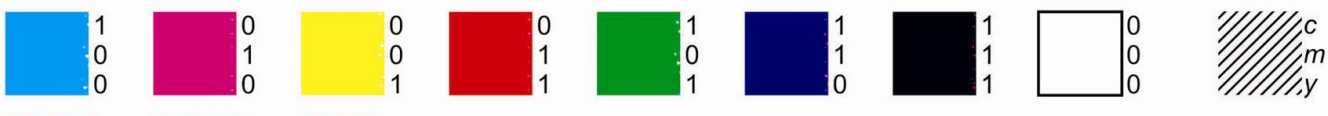

$\mathrm{B}$

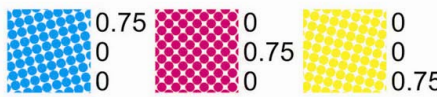

C

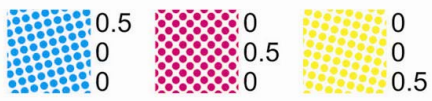

$\mathrm{D}$

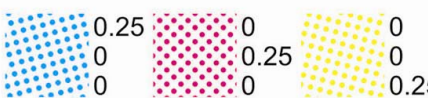

E
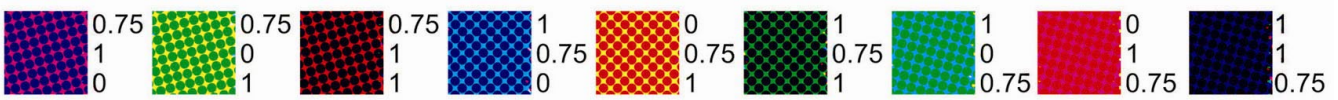

$\mathrm{F}$
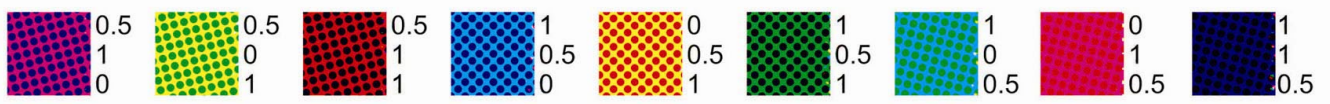

G
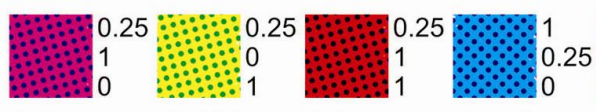

$\because \because \because 001$
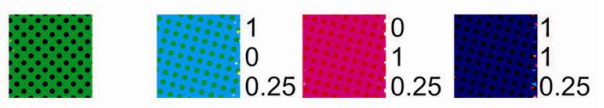

$\mathrm{H}$
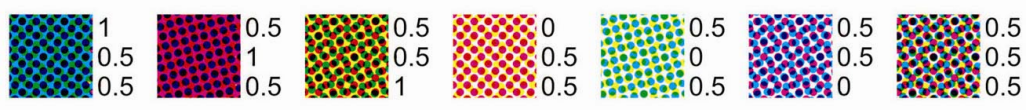

$\begin{array}{rrr}0.75 & 0.25 & 0.25 \\ 0.25 & 0.75 \% & 0.25 \\ 0.25 & 0.25 & 0.75\end{array}$

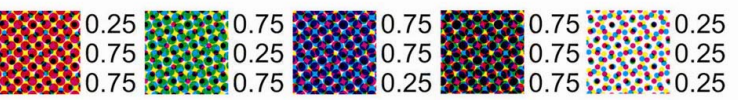

Figure 18: Color patches needed for the calibration of the different models in the case of color halftones printed with cyan, magenta and yellow inks. Cyan, magenta and yellow surface coverages are listed on the right side of each patch.

\section{Independent ink spreading (IIS) method}

Each ink $i$ is printed alone on paper at the nominal surface coverages $a_{i}=0.25,0.5$ and 0.75 , which corresponds to the 9 color patches represented in rows B,C and D of Figure 18. Let us denote as $R_{i}^{\text {meas }}\left(a_{i}, \lambda\right)$ their respective spectral reflectance. These halftones contain two colorants: the ink which should occupy a fractional area $a_{i}$ and the paper white which should occupy the fractional area $1-a_{i}$. Applying the model's equation with these two colorants and these surface coverages should yield a predicted spectral reflectance $R_{i}^{\text {pred }}\left(a_{i}, \lambda\right)$ equal to the measured one. However, due to the fact that the effective ink surface coverage is different from the nominal one, these two reflectances are not the same. We thus fit the effective surface coverage $a_{i}{ }^{\prime}=q$ so as to minimize the deviation between predicted and measured spectra, by quantifying the deviation either by the sum of square differences of the components of the two spectra, i.e. 


$$
a_{i}{ }^{\prime}=\underset{0 \leq q \leq 1}{\arg \min } \sum_{\lambda=380 \mathrm{~nm}}^{730 \mathrm{~nm}}\left[R_{i}^{\text {pred }}(q, \lambda)-R_{i}^{\text {meas }}\left(a_{i}, \lambda\right)\right]^{2}
$$

or by the sum of square difference of the components of their logarithm, i.e.

$$
a_{i}{ }^{\prime}=\underset{0 \leq q \leq 1}{\arg \min } \sum_{\lambda=380 \mathrm{~nm}}^{730 \mathrm{~nm}}\left[\log R_{i}^{\text {pred }}(q, \lambda)-\log R_{i}^{\text {meas }}\left(a_{i}, \lambda\right)\right]^{2}
$$

or by the corresponding color difference given e.g. by the CIELAB $\Delta E_{94}$ metric

$$
a_{i}{ }^{\prime}=\underset{0 \leq q \leq 1}{\arg \min } \Delta E_{94}\left(R_{i}^{\text {pred }}(q, \lambda), R_{i}^{\text {meas }}\left(a_{i}, \lambda\right)\right)
$$

Equation (79) is the most classical way of determining the effective surface coverage. Taking the log of the spectra as in Eq. (80) has the advantage of providing a higher weight to lower reflectance values where the visual system is more sensitive to small spectral differences. Fitting $a_{i}{ }^{\prime}$ from the color difference metric sometimes improves the prediction accuracy of the model in terms of color differences but complicates the optimization. Even at the optimal surface coverage $a_{i}{ }^{\prime}$, the difference between the two spectra is rarely zero and provides a first indication of the prediction accuracy achievable by the model for the corresponding print setup. Once the 9 effective surface coverages are computed, assuming that the effective surface coverage is 0 , respectively 1 , when the nominal surface coverage is 0 (no ink), respectively 1 (full coverage), we obtain three sets of $a_{i}{ }^{\prime}$ values which, by linear interpolation, yield the continuous ink spreading functions $f_{i}$ (Figure 19).
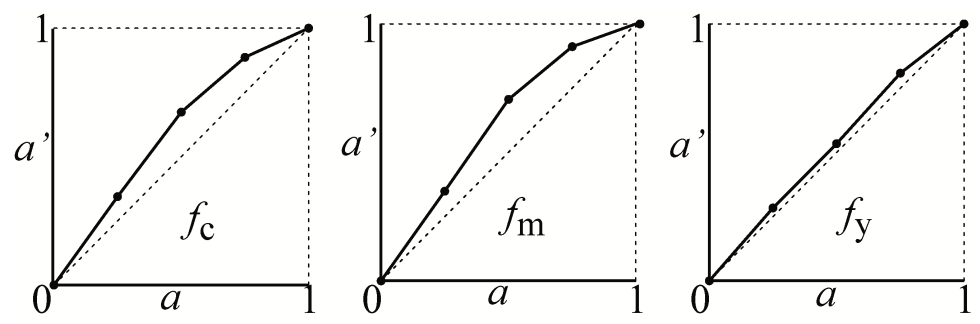

Figure 19. Example of ink spreading curves $f_{i}$ obtained by linear interpolation of the effective surface coverages $a_{i}{ }^{\prime}$ which are deduced from measurement of patches with single-ink halftones (ink $i$ ) printed at nominal surface coverages $0.25,0.5$ and 0.75 (IIS method).

As an alternative, one can print halftones at nominal surface coverage 0.5 only and perform parabolic interpolation (Eq. (71)). The number of patches needed for establishing the ink spreading curves is then reduced to three (row C in Figure 18). 


\section{Superposition-dependent ink spreading (SDIS) method}

As explained in Section 5, one often observes that the amount of ink spreading depends on whether the ink halftone is alone on the support or superposed with other inks. In addition to the effective surface coverages computed from single-ink halftones alone on paper, the SDIS method includes effective surface coverages computed from the single-ink halftones superposed with a solid layer of either one or of the two other inks. With 3 fitted surface coverages per ink spreading curve, 36 color patches need to be printed, represented by the rows $\mathrm{B}$ to $\mathrm{G}$ in Figure 18.
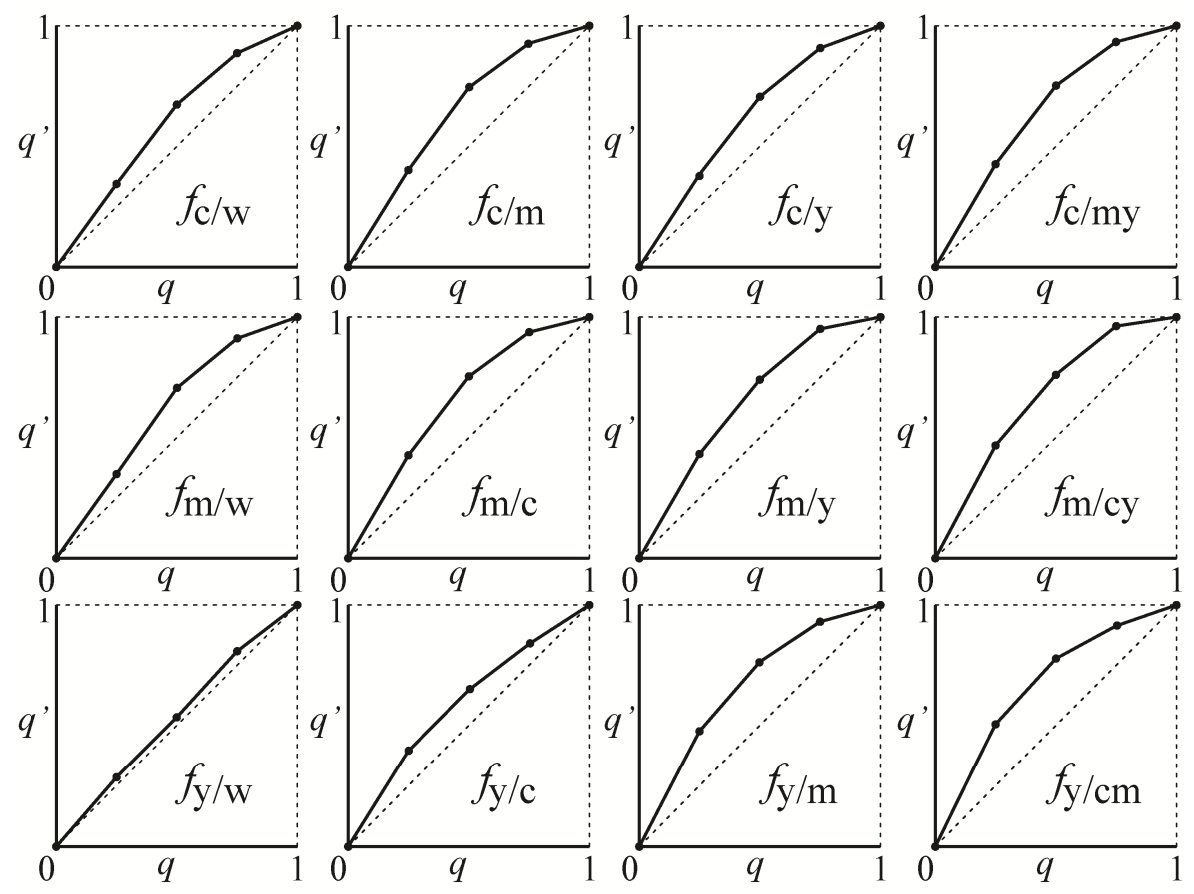

Figure 20. Example of ink spreading curves $f_{i / j}$ obtained by linear interpolation of the effective surface coverages $q_{\mathrm{i} / \mathrm{j}}^{\prime}$ which are deduced from reflectance measurements of patches with single-ink halftones (ink $i$ ) printed at nominal surface coverages $0.25,0.5$ and 0.75 and superposed with a solid layer of colorant $j$ (SDIS method).

The effective surface coverages of the halftones are obtained in the same way as in the IIS method, by considering for each halftone the colorant formed by the halftone dot and the superposed solid inks and the colorant formed by the superposed solid inks only. We obtain 12 sets of effective surface coverages, providing 12 ink spreading curves $q^{\prime}=f_{i / j}(q)$ where $q$ and $q$ ' are respectively the nominal and the effective surface coverages of the ink and where 
subscript $i / j$ denotes ink halftone $i$ superposed with solid colorant $j$ (see Figure 20). By printing and measuring halftones at nominal surface coverage 0.5 only and by performing parabolic interpolation, the required number of patches can be reduced to 12 (rows $\mathrm{C}$ and $\mathrm{F}$ in Figure 18).

\section{Prediction}

Once the spectral reflectances and/or transmittances as well as the ink spreading functions are acquired and/or computed, the model is calibrated. We can then predict the spectral reflectance of halftones for any nominal ink surface coverages $c, m$, and $y$. If the dot gain was calibrated using the independent ink spreading method, the ink spreading functions $f_{i}$ directly provide the effective surface coverages $c^{\prime}, m^{\prime}$, and $y^{\prime}$ of the three inks:

$$
\begin{aligned}
c^{\prime} & =f_{c}(c) \\
m^{\prime} & =f_{m}(m) \\
y^{\prime} & =f_{y}(y)
\end{aligned}
$$

These effective ink surface coverages are plugged into the Demichel equations (3), which provide the effective surface coverages of the eight colorants. The general equation of the model finally predicts the reflectance spectrum of the considered halftone.

If the dot gain was calibrated using the superposition-dependent ink spreading method, the nominal ink surface coverages $c, m$, and $y$ are converted into effective ink surface coverages $c^{\prime}$, $m^{\prime}$ and $y^{\prime}$ by accounting for the superposition-dependent ink spreading. The effective surface coverage of each ink is obtained by a weighted average of the ink spreading curves. The weights are expressed by the surface coverages of the respective colorants on which the ink halftone is superposed. For example, the weight of the ink spreading curve $f_{c}$ (cyan halftone over the white colorant) is proportional to the surface of the underlying white colorant, i.e. $(1-m)(1-y)$. In the case of three halftoned inks, effective surface coverages are obtained by performing a few iterations with Eqs. (75):

$$
\begin{aligned}
& c^{\prime}=\left(1-m^{\prime}\right)\left(1-y^{\prime}\right) f_{c}(c)+m^{\prime}\left(1-y^{\prime}\right) f_{c / m}(c)+\left(1-m^{\prime}\right) y^{\prime} f_{c / y}(c)+m^{\prime} y^{\prime} f_{c / m+y}(c) \\
& m^{\prime}=\left(1-c^{\prime}\right)\left(1-y^{\prime}\right) f_{m}(m)+c^{\prime}\left(1-y^{\prime}\right) f_{m / c}(m)+\left(1-c^{\prime}\right) y^{\prime} f_{m / y}(m)+c^{\prime} y^{\prime} f_{m / c+y}(m) \\
& y^{\prime}=\left(1-c^{\prime}\right)\left(1-m^{\prime}\right) f_{y}(y)+c^{\prime}\left(1-m^{\prime}\right) f_{y / c}(y)+\left(1-c^{\prime}\right) m^{\prime} f_{y / m}(y)+c^{\prime} m^{\prime} f_{y / c+m}(y)
\end{aligned}
$$

For the first iteration, $c^{\prime}=c, m^{\prime}=m$ and $y^{\prime}=y$ are taken as initial values on the right side of the equations. The obtained values of $c^{\prime}, m^{\prime}$ and $y^{\prime}$ are then inserted again into the right side of the equations, which yields new values of $c^{\prime}, m^{\prime}, y^{\prime}$ and so on, until the values of $c^{\prime}, m^{\prime}, y^{\prime}$ 
stabilize. The effective surface coverages of the colorants are calculated by plugging the obtained values for $c^{\prime}, m^{\prime}$ and $y^{\prime}$ into the Demichel equations. The spectral reflectance of the considered halftone is finally provided by the general equation of the model.

\section{Verification}

The prediction accuracy of a model may be verified by comparing predicted and measured spectra on a verification set containing representative printed patches covering the color space. As comparison metric, we use CIELAB $\Delta E_{94}$, obtained by converting the predicted and measured spectra first into CIE-XYZ tristimulus values, calculated with a D65 illuminant and in respect to a $2^{\circ}$ standard observer, and then into CIELAB color coordinates using as white reference the spectral reflectance of the unprinted paper illuminated with the D65 illuminant.

In respect to the calibration patches, the deviation of the spectral predictions from the spectral measurements gives the prediction accuracy of the considered model. Table 4 shows an example issued from samples printed in inkjet at 120 lpi with the Canon Pixma Pro9500 printer on supercalendered paper (patch set $\mathrm{E}$ of Table 6 in the Appendix). The average $\Delta E_{94}$ color differences between measured and predicted spectra obtained with the different models are computed first from the 36 patches used for the calibration of the ink spreading functions and then from the wider set of representative $125 \mathrm{CMY}$ color patches. Even though the average $\Delta E_{94}$ color difference is higher for the 125 patches, the prediction accuracy of the models can already be compared from the 36 calibration patches.

Table 4. Average $\Delta \mathrm{E}_{94}$ between measured and predicted spectral reflectances with different models*

\begin{tabular}{lcc}
\hline Model & $\begin{array}{c}\text { Average } \Delta \mathrm{E}_{94} \text { computed from } \\
36 \text { calibration patches }\end{array}$ & $\begin{array}{c}\text { Average } \Delta \mathrm{E}_{94} \text { computed from } \\
125 \text { halftone patches }\end{array}$ \\
\hline IS-Neugebauer model & 0.92 & 1.13 \\
IS-YNSN model $(n=6)$ & 0.48 & 0.71 \\
IS-CY model & 0.46 & 0.63 \\
IS-WC model & 0.47 & 0.62 \\
\hline
\end{tabular}

*for CMY halftones printed by inkjet at 120 lpi with the Canon Pixma Pro9500 printer on supercalendered paper (patch set E of Table 6 in the Appendix)

Regarding the dot gain calibration method, the superposition-dependant ink spreading method (SDIS) improves considerably the prediction accuracy of the models compared to the independent ink spreading method (IIS). The diagram of Figure 21 shows the prediction accuracies where the coordinates of each point represent the prediction accuracies provided by 
the IIS method (along the abscissa) and SDIS method (along the ordinate). Three models are considered: the Yule-Nielsen modified spectral Neugebauer model, the Clapper-Yule model and the Williams-Clapper model, each one tested on the halftone patch sets listed in the Appendix. Since all points are always below the dotted line of slope 1, we conclude that the SDIS method offers a significantly improved prediction accuracy. In many cases, the prediction accuracy is improved by a factor of 2 to 3 .

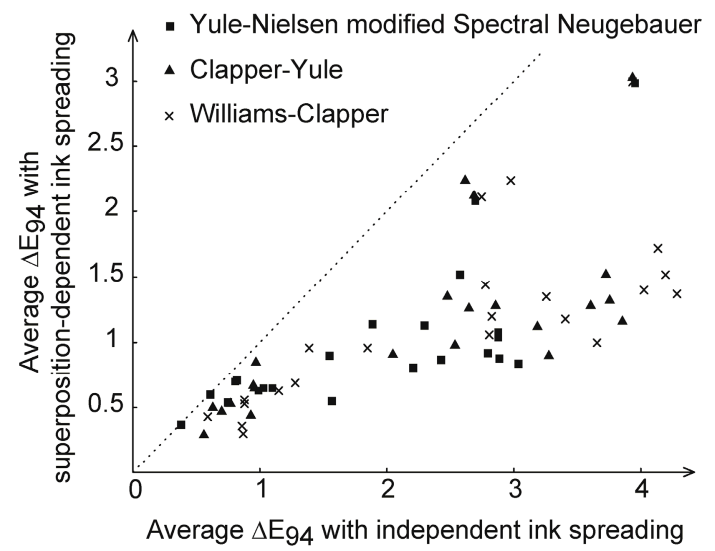

Figure 21: Comparison between the prediction accuracy offered by independent and superposition-dependent ink spreading, for three prediction models, for the sets of halftones listed in the Appendix. The smaller the $\Delta E_{94}$ difference, the higher the prediction accuracy.

\section{Cellular Yule-Nielsen corrected Spectral Neugebauer model}

The CYNSN model is calibrated in a similar manner as the YNSN model but relies on different spectral reflectances and ink spreading functions. We consider for its calibration the 27 spectral reflectances of halftones in which the surface coverage of each ink is $0,0.5$ or 1 (rows $\mathrm{A}, \mathrm{C} \mathrm{F}$ and $\mathrm{H}$ in Figure 18). The ink spreading functions are calibrated from the spectral reflectance of eight halftones in which the surface coverage of the ink halftones are 0.25 and 0.75 (row I in Figure 18). These eight halftones are the centers of the subdomains of the ink surface coverage space represented in Figure 4a. Each of the eight corresponding spectral reflectances enables fitting, in one optimization operation, the three effective surface coverages of respectively the cyan, magenta and yellow inks, which are then converted by quadratic interpolation into ink spreading functions with parabolic shape (Eq. (71)). We thus obtain three ink spreading functions per subdomain, yielding a total of 24 ink spreading functions. 


\section{Four-ink halftones}

All models presented above can be extended to four ink halftones in a straightforward manner, by increasing accordingly the number of spectral parameters and of inks spreading functions. The number of colorants (Neugebauer primaries) becomes $2^{4}=16$ instead of $2^{3}=8$ for 3-ink halftones, see formula (4). This yields 16 spectral reflectances in the SN and YNSN models and 17 for the Clapper-Yule and Williams-Clapper models (16 ink transmittances attached to the 16 colorants as well as the paper's intrinsic reflectance).

The number of ink spreading functions is 4 (one per ink) for the IIS method and 32 (each of the four inks is superposed to the other three inks printed at surface coverages 0 or 1 ) for the SDIS method. Each ink spreading curve is established from three halftones in which one ink is at the nominal surface coverages $0.25,0.5$, and 0.75 . The IIS and SDIS methods therefore need 12 , respectively 96 printed halftones. One may also build the ink spreading functions by parabolic interpolation, which requires one halftone per ink spreading function (ink printed at 0.5 surface coverage superposed with solid layers made of the other inks). This reduces the number of halftones to be printed for the calibration of ink spreading curves to 4 for the IIS method and to 32 for the SDIS method. In the case where the fourth ink is black, the superposition of it with other inks yields black [45]. This reduces the number of ink spreading curves from 32 to 20, see formula (75) and (76).

In order to calibrate a 4-ink prediction model, the spectral parameters and the effective ink surface coverages for creating the ink spreading functions are computed in the same manner as for the corresponding 3-ink prediction model. When predicting reflectances in function of nominal ink surface coverages, one calculates first the corresponding effective ink surface coverages. Then, the surface coverages of the 16 colorants are provided by the 4 -ink Demichel equations (4). In order to predict the reflectance of the considered 4 ink halftone, the effective surface coverages of the colorants, together with the spectral parameters deduced at calibration time are given as input into the prediction model extended to 16 colorants.

The 4-ink CYNSN model relies on $2^{4}=16$ subdomains and $3^{4}=81$ colorants (combinations of inks printed each one at surface coverage $0,0.5$ or 1 ). However, only 16 halftones (one for each subdomain center) are needed to establish the $4 \times 16=64$ ink spreading functions. The ink 
spreading functions are obtained in the same way as for three inks, and predictions are performed by adapting the Demichel equations, i.e. by extending Eq. (15) to 4 inks.

\section{Validity of the calibration}

Regardless of the selected prediction model, the highest prediction accuracy is achieved when the colors are printed on the same support as the calibration patches, with the same inks, the same printing system and the same halftoning method. In addition, one should measure with the same geometry. If one of these requirements is not fulfilled, in order to avoid inaccurate predictions, the whole calibration procedure should be repeated with the modified parameters. The spectral reflectances and transmittances of the models as well as the ink spreading curves account for many complex mechanical and optical phenomena occurring during the interaction of light, inks and paper. This explains why a calibration performed with a given printing support cannot be easily reused for prediction with a different printing support. The development of simplified calibration procedures when either the support or the inks are replaced by different ones is a major challenge for research in the coming years. The two-bytwo centering model (Section 3.4) represents an important progress since, despite requiring a calibration procedure based on a very large number of printed patches, it is valid for any halftoning method.

\section{PERFORMANCE OF THE MODELS}

In order to compare the performances of the prediction models, we carried out several tests with various patch sets printed with different printing systems and ink primaries, on different printing supports at different halftone screen frequencies. These patch sets are described in the Appendix. Prediction accuracy is assessed by the average $\Delta E_{94}$ value computed between spectral reflection predictions and measurements over all the colors of each set, and by the 95percentile indicating the largest deviations, but excluding the worst $5 \%$ predictions which may be due to printing defects or measurement errors. The prediction accuracy of the following models is given in Table 6 (Appendix) for different patch sets:

- Cellular Yule-Nielsen modified Spectral Neugebauer (CYNSN) model,

- Yule-Nielsen modified Spectral Neugebauer (YNSN) model,

- Clapper-Yule model,

- Clapper-Yule model extended to low scattering supports (LSCY),

- Williams-Clapper model extended to halftones. 
All non-cellular models are enhanced by the superposition-dependent ink spreading method presented in Section 5.

\section{General tendencies}

For most tested print sets, the prediction accuracy is excellent since the average color differences are lower than or close to the perceptibility threshold of $\Delta E_{94}=1$. The Cellular Yule-Nielsen modified Spectral Neugebauer model (CYNSN) generally provides the best accuracy. The other models have a comparable accuracy and show similar accuracy variations as the CYNSN model from one set of patches to another. It therefore seems that the prediction accuracy does not much depend on the model but rather on the optical properties of the printing support, the inks and the printing process.

Best predictions are obtained when the inks are weakly absorbing, as in set $\mathrm{F}$ where the ink thickness is one quarter of the maximal ink thickness allowed by the inkjet printer, or sets $\mathrm{D}, \mathrm{E}$, $\mathrm{C}, \mathrm{L}$ to $\mathrm{O}$ and $\mathrm{R}$ to $\mathrm{V}$ where the relative ink thickness was $1 / 2$ (i.e. half the maximal allowed thickness). Ink thickness has a direct influence on the absorbance of the halftone. High absorbance emphasizes the optical dot gain as well as secondary optical effects. A second condition for a successful prediction is to have nonscattering inks on top of a strongly diffusing support. Scattering by the inks is often an issue with electro-photographic printing: the average $\Delta E_{94}$ value obtained for set $\mathrm{A}$ is higher than the one obtained for set $\mathrm{D}$ printed in inkjet or set $\mathrm{W}$ printed in offset. But the issue of low scattering by the printing support is more problematic. None of the models manages to predict correctly the spectral reflectance of colors printed on tracing paper which is very weakly scattering (sets P and Q).

\section{Performance of the surface color prediction models}

For most of the tested print sets presented in Table 6 (Appendix) the best accuracy is provided by the Cellular Yule-Nielsen modified Spectral Neugebauer model (CYNSN), followed by the Yule-Nielsen modified Spectral Neugebauer model (SDIS-YNSN). The surface color prediction models, presented in Section 3, have the advantage to be directly based on measured spectra and therefore to incorporate all optical phenomena taking place within the inks, for example the retro-reflection of light by the pigments. The non-linear interpolation of these measured spectra is based on the free parameter $n$, which is correlated with the halftone screen frequency and therefore represents a measure of light propagation between neighboring ink halftones due to multiple reflections and lateral light scattering. However, it may also be 
influenced by further phenomena, such as thickness variation of the ink dot profile [46]. When the halftone screen frequency is increased, the fitted optimal $n$ value increases. This appears clearly when comparing sets $\mathrm{O}, \mathrm{P}, \mathrm{Q}$, and $\mathrm{R}$, sets $\mathrm{L}$ and $\mathrm{M}$ or sets $\mathrm{V}$ and $\mathrm{W}$ in Table 6.

In order to better understand the capacity of the SDIS-YNSN model to fit the actual reflectances of a halftone, we establish a relationship between the attenuation of light due to the ink halftone, no attenuation by the ink and maximal attenuation by the corresponding solid ink. For this purpose, by denoting as $R_{0}, R_{a}$ and $R_{1}$ the spectral reflectances of patches where the ink has the respective surface coverages of $0, a$ and 1, we propose to convert the Yule-Nielsen equation written for single ink halftones

$$
R_{a}(\lambda)=\left[(1-a) R_{0}^{1 / n}(\lambda)+a R_{1}^{1 / n}(\lambda)\right]^{n}
$$

into the following equation, obtained by dividing equation (84) by $R_{0}$

$$
R_{a} / R_{0}=\left[1-a+a\left(R_{1} / R_{0}\right)^{1 / n}\right]^{n}
$$

This relation of the form $R_{a} / R_{0}=f_{a}\left(R_{1} / R_{0}\right)$ can be verified experimentally from the measurements of $R_{0}, R_{a}$ and $R_{1}$, which provide as many points as values contained in the measured spectra. This relation also enables observing, for the fitted values of $a$ and $n$, the variation of $R_{a} / R_{0}$ as a function of $R_{1} / R_{0}$ from 0 (reflectance $R_{1}$ is zero, which means that all light is absorbed by the ink) to 1 ( $R_{1}$ is equal to $R_{0}$, which means that the ink is transparent).

Figure 22 shows the example of a cyan ink halftone printed on Canon MP101 mat paper at 0.5 nominal surface coverage and 0.5 relative ink thickness with the Canon Pixma Pro9500 inkjet printer. The spectral reflectances are measured with diffuse- $8^{\circ}$ geometry, specular reflection included. On the right of the figure, the measured spectra of $R_{0}, R_{a}$ and $R_{1}$ are plotted as well as the predicted spectrum $\hat{R}_{a}$ for $n=10$ and for the $a$ value fitted so as to minimize the deviation from the measured spectrum. On the left of the figure, the theoretical curve $\hat{R}_{a} / R_{0}=f_{a}\left(R_{1} / R_{0}\right)$ is plotted in solid black line for the same fitted value of $a$ and the same $n$. The red dashed straight line shows how it would vary with this value of $a$ and with $n=1$, i.e. according to the Spectral Neugebauer model. The black squares correspond to the points $\left(R_{1} / R_{0}, R_{a} / R_{0}\right)$ derived from measurements. 

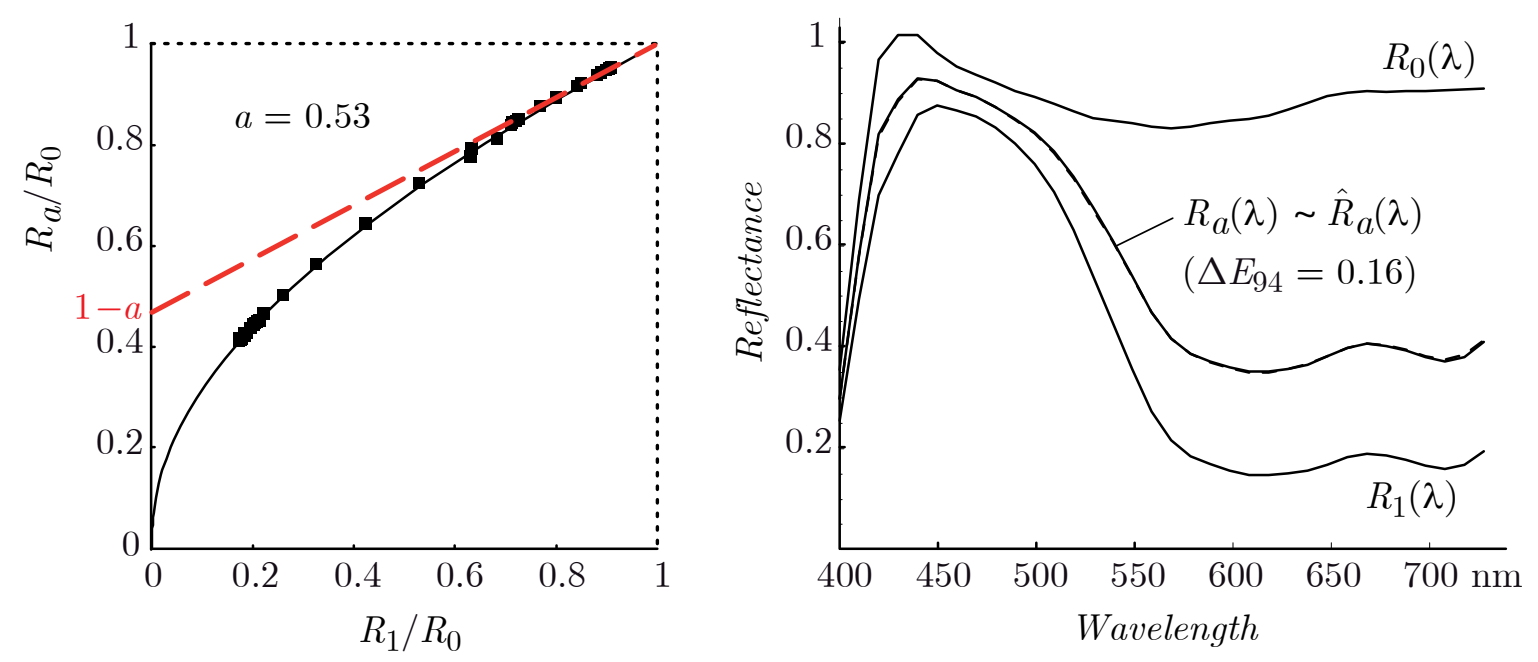

Figure 22: Comparison of predicted and measured spectral reflectances of cyan ink printed at 0.5 nominal surface coverage. Left: diagram representing the curve predicted according to equation (85) (black solid curve) and the measured points $\left(R_{1} / R_{0}, R_{a} / R_{0}\right)$ at the different wavelengths. The red dashed line represents the reflectance that would be obtained if the halftone would behave as predicted by the simple Spectral Neugebauer model. Right: measured spectral reflectances of the unprinted paper $R_{0}(\lambda)$, of the halftone $R_{a}(\lambda)$ and of the solid ink patch $R_{1}(\lambda)$ as well as predicted reflectance $\hat{R}_{a}(\lambda)$ of the halftone.

In this example, we observe an excellent agreement between prediction and measurement. Function $f_{a}$ models perfectly the nonlinear relationship between the reflectances of the halftone, the unprinted paper, and the paper fully covered by cyan ink. However, such an agreement between prediction and measurement cannot be reached for all types of prints: it may occur that no function $f_{a}$ of the form $x \mapsto\left(1-a+a x^{1 / n}\right)^{n}$ coincides with the points $\left(R_{1} / R_{0}, R_{a} / R_{0}\right)$ deduced from reflectance measurements, even for optimal values of $n$ and $a$. In such a case, the prediction accuracy provided by the Yule-Nielsen spectral Neugebauer model will be poor. However, the prediction accuracy may be significantly improved by using the Cellular Yule-Nielsen spectral Neugebauer (CYNSN) model. Since the single ink halftones at surface coverage 0.5 are considered as base colorants, the model gains spectral information relative to ink halftones which was not contained within the spectral reflectance of the solid colorant patches. This explains why the CYNSN model is almost always more accurate than the other models (see for example sets A to K in Table 6, Appendix). 


\section{Performance of the phenomenological prediction models}

The Clapper-Yule model and the Williams-Clapper model extended to halftones assume that the lateral propagation of light within the paper bulk is larger than the halftone dot period. They are therefore theoretically restricted to halftones with high screen frequency. For example, if we compare sets $\mathrm{V}$ and $\mathrm{W}$ printed with the same offset press on the same paper but with different screen frequencies, respectively 30 and 60 lines per $\mathrm{cm}$ (76 and 152 lpi), predictions are better for the set W. However, the experience shows that they may also perform well for middle and low screen frequencies (see for example set $\mathrm{L}$ where the halftone screen frequency is 90 lpi). Sets $\mathrm{R}$ to $\mathrm{U}$, which were produced in the same way with different screen frequencies, are a counterexample: the prediction accuracy decreases as the screen frequency increases. As in the case of the Yule-Nielsen modified spectral Neugebauer model (SDIS-YNSN model), other factors such as the absorbance of the ink or the non-uniformity of the dot profile may in some cases have more impact on the prediction accuracy than the screen frequency.

Table 5. Prediction accuracy of models calibrated from measurements based on the $45^{\circ}: 0^{\circ}$ geometry for different prediction geometries

\begin{tabular}{lcc}
\hline Prediction geometry: & $45^{\circ}: 0^{\circ}$ geometry & de: $8^{\circ}$ geometry \\
\hline SDIS-Clapper-Yule model & $0.75(1.48)^{*}$ & $1.07(2.16)$ \\
SDIS-Williams-Clapper model & $0.89(1.71)$ & $1.17(2.28)$ \\
SDIS-YNSN model $(n=10)$ & $0.52(1.17)$ & $1.45(3.26)$ \\
\hline
\end{tabular}

*Average $\Delta \mathrm{E}_{94}(95$-quantile)

One advantage of the phenomenological models is the possibility to adapt the Fresnel terms to the measuring geometry used for measurements. This is particularly interesting when we make predictions for a geometry different from the one used for calibration. We tested this possibility with the 125 halftones printed in inkjet on Canon PP201 glossy paper (set K), by calibrating both the Clapper-Yule and Williams-Clapper models (with the superposition-dependent ink spreading method) from measurements based on the $45^{\circ}: 0^{\circ}$ geometry and making the predictions for the de: $8^{\circ}$ geometry. The predicted spectra are compared with spectra measured with the de: $8^{\circ}$ geometry. The results are given in Table 5. By way of comparison, we also tested the capacity of the Yule-Nielsen modified Spectral Neugebauer model enhanced by superposition-dependent ink spreading, calibrated with the $45^{\circ}: 0^{\circ}$ geometry to predict the spectral reflectances observed with a de: $8^{\circ}$ geometry. Since this latter model provides no way of accounting for a change in measuring geometry, it is less accurate than the Clapper-Yule and 
Williams-Clapper models despite the fact that it is more accurate when the same geometry is used both for calibration and for prediction.

\section{CONCLUSIONS}

This contribution presents the state of the art mainstream models capable of predicting the reflectance of halftone prints. These models are capable of predicting with a high accuracy the reflectance of multi-ink halftones on a diffuse substrate. They support both low and highfrequency screens produced according to different halftoning methods. However, model calibration should be performed with the same inks, same halftones and same substrate as the inks, halftones and substrate used for reflectance prediction. Only the two-by-two spectral prediction model calibrated with a given set of inks and substrate is able to predict reflectances independently of the halftone screen.

Among the cited prediction models one may have to decide which model to choose. The prediction accuracy of each model can be checked on a set of calibration and test patches. Generally, one may test the prediction accuracy of a model by printing as many patches as combinations of ink surface coverage of $0,0.250 .50 .75$ and 1 . When one needs to account for different illumination and capturing geometries, phenomenological models such as the Clapper-Yule and the Williams-Clapper models are more adequate. These models also allow to predict the change in reflectances as a function of ink thickness variations. For completely transparent colorant layers, i.e. colorants transmitting light without scattering effects, the Williams-Clapper is theoretically more accurate than the Clapper-Yule model. However, in printing systems using slightly scattering inks, there is not much difference between the prediction accuracy of these two models. The ink spreading enhanced cellular Yule-Nielsen spectral prediction model (IS-CYNSN) offers the highest prediction accuracy. This is due to the

fact that it is calibrated with pseudo-colorant reflectance measurements at all combinations of $0,50 \%$ and $100 \%$ ink surface coverages. It can therefore better account for halftones within the color prints.

By applying optimization techniques, the presented prediction models may also be used to fit nominal ink surface coverages in order to obtain a given color. For this purpose, one may convert a predicted reflectance spectrum to a predicted color, e.g. in the CIELAB color space, and vary the ink surface coverages so as to minimize a difference metric between predicted and 
desired color. In the case of 4 inks, fitting ink surface coverages in order to obtain a given color is an underdetermined problem. Further criteria should be introduced, such as minimizing the contrast within the halftone dots [2], minimizing the usage of inks [1] or minimizing a metameric index [3]. An alternative is to reformulate the problem and try to minimize a difference metric between desired and predicted reflectance spectra [48], [49].

The presented mainstream prediction models rely on the following hypotheses:

- interface reflections and refractions are independent of wavelength, i.e. the media are assumed to be non-dispersive and to have a constant real refractive index over the wavelengths of the visible spectrum of light,

- the substrate is strongly diffusing and only slightly absorbing (Lambertian intrinsic reflectance),

- different wavelengths do not influence one another (e.g. fluorescence phenomena are not accounted for),

- Interferential and diffractive phenomena have only a very minor impact on the reflected or transmitted light

Clearly, these hypotheses are not always fulfilled. For example, rough substrates or small particles within the ink layers may introduce interference phenomena. Some substrates may not have a Lambertian intrinsic reflectance. Accounting for these phenomena is a challenge for future research.

A further research challenge resides in characterizing separately the substrate, the inks and the interaction between inks and substrate and using these characterizations to predict the reflectance of the substrate printed with the corresponding inks.

\section{Acknowledgments}

The authors would like to thank Romain Rossier, Jacques Machizaud as well as Baptiste Paul for their contribution to the experimental results presented in this contribution. Thanks to Vahid Babaei and Li Yang who performed a thorough review. We also thank the Swiss National Science Foundation for their support (grant 200021_143501). 


\section{REFERENCES}

1. Morovič, J, Morovič, P, Arnabat, J. (2010) HANS - A new color separation and halftoning paradigm. Proceedings IS\&T 18th Color Imaging Conference, 359-364.

2. S. Chosson, S, Hersch RD. (2001) Visually-based color space tetrahedrizations for printing with custom inks, Conf. Color Imaging: Device-Independent Color, Color Hardcopy, and Graphic Arts VI, SPIE Vol. 4300, 81-92.

3. Tzeng, DY, Berns, RS. (2000) Spectral-based six-color separation minimizing metamerism, Proceedings IS\&T 8th Color Imaging Conference, 342-347.

4. Demichel, M.E. (1924) Procédé 26, 17-21.

5. Amidror I, Hersch, RD. (2000) Neugebauer and Demichel: dependence and independence in n-screen superpositions for colour printing, Color Research and Application 25, $267-$ 277.

6. Neugebauer, HEJ. (1937) "Die theoretischen Grundlagen des Mehrfarbendrucks", Zeitschrift fuer wissenschaftliche Photographie 36, 36-73, translated by Wyble, D. and Kraushaar A, in "The theoretical basis of multicolor letterpress printing," Color Research and Application 30, (2005) 323-331.

7. Murray, A. (1936) Monochrome reproduction in photoengraving, J. Franklin Institute, Vol. 221, 721-724.

8. ISO 12647-3:2005. Graphic technology - Process control for the production of half-tone colour separations, proofs and production prints - Part 3: Coldset offset lithography on newsprint.

9. DIN 16536-2:1995. Testing of prints and printing inks in graphic technology - Colour density measurements on on-press or off-press prints - Part 2: Instrument specifications for reflection densitometers and their calibration.

10. Kang, HR. (1994) Applications of color mixing models to electronic printing. Journal of Electronic Imaging 3, 276-287.

11. Yule, JAC, Nielsen, WJ. (1951) The penetration of light into paper and its effect on halftone reproduction, TAGA Proceedings, 65-66.

12. Viggiano, JAS. (1990) Modeling the Color of Multi-Colored Halftones, TAGA Proceedings, 44-62.

13. Heuberger, KJ, Jing, ZM, and Persiev S. (1992) Color Transformations and lookup tables, in TAGA/ISCC Proceedings, 863-881. 
14. Balasubramanian R. (1999) Optimization of the spectral Neugebauer model for printer characterization, Journal of Electronic Imaging 8, 156-166,

15. Agar, U, Allebach, JP. (1998) An iterative cellular YNSN method for color printer characterization, Proceedings IS\&T 6th Color Imaging Conference, 197-200,

16. Rossier, R, Bugnon, T, Hersch, RD. (2010) Introducing ink spreading within the cellular Yule-Nielsen modified Neugebauer model, Proceedings IS\&T $18^{\text {th }}$ Color Imaging Conference, 295-300.

17. Pappas, TN. (1999) Least-Squares Model-Based Halftoning, IEEE Transactions on Image Processing 8, 1102-1116.

18. Wang, SG. (1998) Two-by-Two Centering Printer Model with Yule-Nielsen Equation, IS\&T International Conference on Digital Printing Technologies, 302-305.

19. Wang, SG. (1999) Feedback for printer Color Calibration, IS\&T International Conference on Digital Printing Technologies, 327-330.

20. Babaei, V, Rossier, R, Hersch, RD. (2012) Reducing the number of calibration patterns for the two-by-two dot centering model, Conf. Color Imaging XVII: Displaying, Processing, Hardcopy, and Applications, SPIE Vol. 8292, paper 829208, 1-9

21. Clapper, FR, Yule, JAC. (1953) The Effect of Multiple Internal Reflections on the Densities of Halftone Prints on Paper, Journal of the Optical Society of America 43, 600603.

22. Williams, FC, Clapper, FR. (1953) Multiple Internal Reflections in Photographic Color Prints, Journal of the Optical Society of America 29, 595-599.

23. Arney, JS. (1997) A Probability Description of the Yule-Nielsen Effect I, Journal of Imaging Science and Technology 41, 633-636

24. Rogers, GL. (1998) Effect of light scatter on halftone color, Journal of the Optical Society of America A 15, 1813-1821.

25. Yang, L, Lenz, L, Kruse, B. (2001) Light scattering and ink penetration effects on tone reproduction, Journal of the Optical Society of America A 18, 360-366.

26. Sormaz, M, Stamm, T, Mourad, S, and Jenny P. (2009) Stochastic modeling of light scattering with fluorescence using a Monte Carlo-based multiscale approach, Journal of the Optical Society of America A 26, 1403-1413

27. Kubelka, P. (1954) New contributions to the optics of intensely light-scattering material, part II: Non-homogeneous layers. Journal of the Optical Society of America 44, 330-335

28. Emmel, P. (2003) Physical models for color prediction, in Digital Color Imaging, (Ed. G. Sharma), CRC Press, 173-238 
29. Hébert, M, Hersch, RD. (2006) Reflectance and transmittance model for recto-verso halftone prints, Journal of the Optical Society of America A 23, 2415-2432

30. Hébert, M, Hersch, RD, Becker, JM. (2007) Compositional reflectance and transmittance model for multilayer specimens, Journal of Optical Society of America A 24, 2628-2644.

31. Kubelka, P. (1948) New Contributions to the Optics of Intensely Light-Scattering Materials, Part I, Journal of Optical Society of America 38, 448-457.

32. Nobbs, JH. (1985) Kubelka-Munk theory and the prediction of reflectance, Review of Progress in Coloration and Related Topics 15, 66-75

33. Yang, L, Kruse, B. (2000) Ink penetration and its effects on printing, Conf. Color Imaging: Device-Independent Color, Color Hardcopy and Graphic Arts V, SPIE Vol. 3963, 365-375.

34. Judd, DB. (1942) Fresnel reflection of diffusely incident light, Journal of Research of the National Bureau of Standards 29, 329-332.

35. Saunderson, JL. (1942) Calculation of the Color of Pigmented Plastics, Journal of Optical Society of America 32, 727-736.

36. Rogers, G. (2000) A Generalized Clapper-Yule Model of Halftone Reflectance. Journal of Color Research and Application 25, 402-407

37. Hersch, RD, Emmel, P, Crété, F, Collaud F. (2005) Spectral reflection and dot surface prediction models for color halftone prints, Journal of Electronic Imaging 14, paper 33001, $1-12$.

38. Shore, JD, Spoonhower, JP. (2001) Reflection Density in Photographic Color Prints: Generalizations of the Williams-Clapper Transform, Journal of Imaging Science and Technology 45, 484-488.

39. Hebert, M, Hersch, RD. (2004) Classical Print Reflection Models: A Radiometric Approach, Journal of Imaging Science and Technology 48, 363-374.

40. Yule, JAC, Colt, R. (1951) Colorimetric investigations in multicolor printing, TAGA Proceedings 77- 82

41. Viggiano, JAS. (1983) The GRL* Dot Gain Model, TAGA Proceedings, 423-439.

42. Xia, M, Saber, E, Sharma, G, Tekalp, M. (1999) End to end color printer calibration by total least squares regression, IEEE Transactions On Image Processing 8, 700-716.

43. Iino, K, Berns, RS. (1998) Building color management modules using linear optimization II. Prepress system for offset printing, Journal of Imaging Science and Technology 42, 99114

44. Hersch, RD. Crété, F. Improving the Yule-Nielsen modified spectral Neugebauer model by dot surface coverages depending on the ink superposition conditions, Electronic Imaging 
Symp., Conf. Color Imaging X: Processing, Hardcopy and Applications, SPIE Vol. 5667, 434-445 (2005)

45. Bugnon, Th, Brichon M, and Hersch, RD. (2008) Simplified Ink Spreading Equations for CMYK Halftone Prints, Color Imaging XIII: Processing, Hardcopy, and Applications, SPIE Vol. 6807, paper 680717, 1-12

46. Hébert, M, Hersch, RD. (2010) Analyzing halftone dot blurring by extended spectral prediction models, Journal of Optical Society of America A 27, 6-12

47. Emmel, P, Hersch, RD. (2002) Modeling Ink Spreading for Color Prediction, Journal of Imaging Science and Technology 46, 237-246.

48. Bugnon, Th, Brichon M, and Hersch, RD. (2007) Model-Based Deduction of CMYK Surface Coverages from Visible and Infrared Spectral Measurements of Halftone Prints, Color Imaging XII: Processing, Hardcopy, and Applications, SPIE Vol. 6493, paper 649310, 1-10.

49. Urban, P, Berns, RS. (2011) Paramer mismatch-based spectral gamut mapping, IEEE Transactions on Image Processing 20, 1599-1610.

\section{APPENDIX}

Set A: 125 patches printed at 100 lpi on Canon MP101 $170 \mathrm{~g} / \mathrm{m} 2$ mat paper with the Xerox Phaser 6360DN electrophotographic printer with Cyan, Magenta and Yellow inks; measured with the $45^{\circ}: 0^{\circ}$ geometry.

Set B: 125 patches printed at 100 lpi on Canon MP101 $170 \mathrm{~g} / \mathrm{m} 2$ mat paper with the Canon Pixma Pro9500 Mark II inkjet printer with Cyan, Magenta and Yellow inks at maximal thickness ${ }^{1}$; measured with the di: $8^{\circ}$ geometry (i.e. the diffuse-eight degree geometry with specular component included).

Set $C$ : Same as B, printed with a relative ink thickness $1 / 2$.

Set D: 125 patches printed at 120 lpi on Canon MP101 $170 \mathrm{~g} / \mathrm{m} 2$ mat paper with the Canon Pixma Pro9500 inkjet printer with Cyan, Magenta and Yellow inks of relative thickness 1/2; measured with the di: $8^{\circ}$ geometry.

Set E: Same as set D, printed on supercalendered nonfluorescing APCO-II paper from Scheufelen Company, Germany.

Set F: Same as set B, printed on the APCO-II paper with relative ink thickness 1/4.

\footnotetext{
1 The Canon Pixma Pro9500 and Canon Pixma Pro9500 Mark II printers enable printing at different ink thicknesses by modifying the number of ink droplets deposited on each pixel of the printed image.
} 
Set $G$ : Same as set B, printed on HP Premium $240 \mathrm{~g} / \mathrm{m} 2$ glossy paper.

Set H: Same as set B, printed on common $80 \mathrm{~g} / \mathrm{m} 2$ office paper.

Set I: 125 patches printed at 150 lpi on Canon PP201 $260 \mathrm{~g} / \mathrm{m} 2$ glossy coated paper, with Cyan, Magenta and Yellow inks; measured with the di: $8^{\circ}$ geometry.

Set J: Same as set I, measured with the de: $8^{\circ}$ geometry (diffuse-eight degree geometry with specular component excluded).

Set $K$ : Same as set L, measured with the $45^{\circ}: 0^{\circ}$ geometry.

Set L: 40 patches printed at 90 lpi on the APCO-II paper with the Canon Pixma Pro9500 inkjet printer with Cyan, Magenta and Yellow inks at relative ink thickness 1/2; measured with the di: $8^{\circ}$ geometry.

Set M: Same as set L, printed at 150 lpi.

Set N: 40 patches printed at 120 lpi on common office paper $80 \mathrm{~g} / \mathrm{m} 2$ with the Canon Pixma Pro9500 inkjet printer with Cyan, Magenta and Yellow inks at relative ink thickness 1/2; measured with the di: $8^{\circ}$ geometry.

Set $O$ : Same as set $\mathrm{N}$, printed on Biotop non-fluorescent noncalendered $80 \mathrm{~g} / \mathrm{m}^{2}$ paper, a paper being noticeably porous.

Set $P$ : Same as set $\mathrm{N}$, printed on Canson $90 \mathrm{~g} / \mathrm{m}^{2}$ tracing paper.

Set $Q$ : Same as set $\mathrm{P}$, printed at 75 lpi.

Set R: 729 patches printed at 50 lpi on coated paper with a Canon IP4000 inkjet printer, with Cyan, Magenta and Yellow inks; measured with the $45^{\circ}: 0^{\circ}$ geometry.

Set $S$ : Same as set R, printed at 75 lpi.

Set T: Same as set $\mathrm{R}$, printed at 100 lpi.

Set $U$ : Same as set $\mathrm{R}$, printed at 125 lpi.

Set V: 729 patches printed at 75 lpi on coated paper with a Komori offset press, with Cyan, Magenta and Yellow inks; measured with the $45^{\circ}: 0^{\circ}$ geometry.

Set $W$ : Same as set V, printed at 150 lpi.

Set X: 625 patches printed at 100 lpi on Canon MP101 $170 \mathrm{~g} / \mathrm{m} 2$ mat paper with the Canon IP4000 inkjet printer with Cyan, Magenta, Yellow, and Black inks; measured with the $45^{\circ}: 0^{\circ}$ geometry.

Set Y: 625 patches printed at 120 lpi on Canon MP101 $170 \mathrm{~g} / \mathrm{m} 2$ mat paper with the Canon Pixma Pro9500 inkjet printer with Cyan, Magenta, Yellow and custom Green inks at relative ink thickness $1 / 2$; measured with the $45^{\circ}: 0^{\circ}$ geometry.

Set Z: Same as set Y, printed with Blue, Orange, Yellow and Magenta inks at relative ink thickness $1 / 2$. 
Table 6. Color differences between spectral reflectance measurements and predictions, with superposition dependent ink spreading (SDIS) except for CYNSN.

\begin{tabular}{|c|c|c|c|c|c|c|c|c|c|c|c|c|c|c|c|c|c|}
\hline \multirow{3}{*}{$\begin{array}{c}\text { Label } \\
\mathrm{A}\end{array}$} & \multirow{2}{*}{\multicolumn{5}{|c|}{$\begin{array}{l}\text { Printer, Paper, Patch number / Ink set, Screen } \\
\text { frequency, Measuring geometry }\end{array}$}} & \multirow{2}{*}{\multicolumn{2}{|c|}{\begin{tabular}{|c|} 
CYNSN \\
average $(95-Q)$
\end{tabular}}} & \multicolumn{3}{|c|}{ YNSN } & \multicolumn{3}{|c|}{ LSCY } & \multirow{2}{*}{\multicolumn{2}{|c|}{$\begin{array}{l}\text { Clapper-Yule } \\
\text { average }(95-Q)\end{array}$}} & \multirow{2}{*}{\multicolumn{2}{|c|}{$\begin{array}{c}\text { Williams-Clapper } \\
\text { average }(95-Q)\end{array}$}} \\
\hline & & & & & & & & \multirow{2}{*}{$\begin{array}{c}n \\
6.4\end{array}$} & \multicolumn{2}{|c|}{ average $(95-Q)$} & \multirow{2}{*}{$\begin{array}{l}b \\
0\end{array}$} & \multicolumn{2}{|c|}{ average $(95-Q)$} & & & & \\
\hline & Laserjet & MP101 & 125 / CMY & 100 lpi & $45^{\circ}: 0^{\circ}$ & 0.89 & $(2.14)$ & & 1.04 & (2.23) & & 1.28 & $(2.48)$ & 1.28 & (2.48) & 1.4 & (2.74) \\
\hline $\mathrm{B}$ & Inkjet $^{\mathrm{a}}$ & MP101 & 125 / CMY & 100 lpi & $\mathrm{di}: 8^{\circ}$ & 0.56 & (1.38) & 10 & 1,14 & $(2,42)$ & 0 & 1.35 & (3.42) & 1.35 & (3.42) & 1.39 & (3.53) \\
\hline $\mathrm{C}$ & Inkjet $^{c}$ & MP101 & $125 / \mathrm{CMY}$ & 100 lpi & $\mathrm{di}: 8^{\circ}$ & 0.15 & $(0.50)$ & 5 & 0,22 & $(0,49)$ & 0.1 & 0.27 & $(0.60)$ & 0.32 & $(0.71)$ & 0.41 & $(0.78)$ \\
\hline $\mathrm{D}$ & Inkjet $^{\mathrm{c}}$ & MP101 & $125 / \mathrm{CMY}$ & 120 lpi & $\mathrm{di}: 8^{\circ}$ & 0.23 & $(0.63)$ & 10 & 0.37 & $(0.93)$ & 0 & 0.33 & $(0.71)$ & 0.33 & $(0.71)$ & 0.33 & $(0.61)$ \\
\hline $\mathrm{E}$ & Inkjet $^{c}$ & APCO & $125 / \mathrm{CMY}$ & 120 lpi & $\mathrm{di}: 8^{\circ}$ & 0.20 & $(0.64)$ & 4.6 & 0.71 & $(1.80)$ & 0.2 & 0.66 & (1.54) & 0.65 & (1.56) & 0.63 & $(1.52)$ \\
\hline $\mathrm{F}$ & Inkjet $^{d}$ & APCO & $125 / \mathrm{CMY}$ & 100 lpi & di: $8^{\circ}$ & 0.21 & (0.53) & 3 & 0,33 & $(0,78)$ & 0.5 & 0.32 & $(0.77)$ & 0.38 & $(0.76)$ & 0.41 & $(0.80)$ \\
\hline G & Inkjet $^{\mathrm{a}}$ & HPglossy & $125 / \mathrm{CMY}$ & 100 lpi & $\mathrm{di}: 8^{\circ}$ & 0.64 & $(1.65)$ & 7,3 & 1,52 & $(3,14)$ & 0 & 2.06 & (4.13) & 2.06 & (4.13) & 2.19 & (4.35) \\
\hline $\mathrm{H}$ & Inkjet $^{\mathrm{a}}$ & Office & $125 / \mathrm{CMY}$ & 100 lpi & $\mathrm{di}: 8^{\circ}$ & 0.70 & (1.94) & 10 & 1,08 & $(2,98)$ & 0 & 1.12 & $(2.89)$ & 1.12 & (2.89) & 1.18 & (2.94) \\
\hline I & Inkjet $^{b}$ & PP201 & $125 / \mathrm{CMY}$ & 150 lpi & di: $8^{\circ}$ & 0.38 & $(0.98)$ & 10 & 0.89 & (1.94) & 0 & 0.92 & (1.96) & 0.92 & (1.96) & 1.01 & $(0.92)$ \\
\hline $\mathrm{J}$ & Inkjet $^{b}$ & PP201 & $125 / \mathrm{CMY}$ & 150 lpi & de: $8^{\circ}$ & 0.35 & $(0.81)$ & 3.8 & 0.67 & (1.42) & 0 & 0.93 & $(1.86)$ & 0.93 & (1.86) & 1.06 & (2.13) \\
\hline $\mathrm{K}$ & Inkjet $^{b}$ & PP201 & $125 / \mathrm{CMY}$ & 150 lpi & $45^{\circ} / 0^{\circ}$ & 0.42 & $(1.05)$ & 3.4 & 0.52 & (1.17) & 0 & 0.75 & $(1.48)$ & 0.75 & (1.48) & 0.89 & (1.71) \\
\hline $\mathrm{L}$ & Inkjet $^{c}$ & APCO & $40 / \mathrm{CMY}$ & 90 lpi & $\mathrm{di}: 8^{\circ}$ & & & 4 & 0.55 & (1.11) & 0.2 & 0.49 & $(0.71)$ & 0.47 & (0.83) & 0.53 & $(0.85)$ \\
\hline $\mathrm{M}$ & Inkjet $^{c}$ & APCO & 40 / CMY & 150 lpi & $\mathrm{di}: 8^{\circ}$ & & & 7 & 0.65 & (1.11) & 0.1 & 0.51 & (1.06) & 0.53 & (1.10) & 0.56 & (1.16) \\
\hline $\mathrm{N}$ & Inkjet $^{c}$ & Office & 40 / CMY & 120 lpi & $\mathrm{di}: 8^{\circ}$ & & & 10 & 0.70 & (1.13) & 0 & 0.50 & $(0.83)$ & 0.5 & $(0.83)$ & 0.43 & $(0.60)$ \\
\hline $\mathrm{O}$ & Inkjet $^{c}$ & Biotop & 40 / CMY & 120 lpi & $\mathrm{di}: 8^{\circ}$ & & & 10 & 0.65 & (1.12) & 0 & 0.44 & (0.74) & 0.44 & $(0.74)$ & 0.36 & $(0.47)$ \\
\hline$P$ & Inkjet $^{\mathrm{c}}$ & Tracing & 40 / CMY & 120 lpi & $\mathrm{di}: 8^{\circ}$ & & & 10 & 2.99 & (4.58) & 0 & 3.03 & (4.66) & 3.03 & (4.66) & 3.00 & (4.64) \\
\hline Q & Inkjet $^{c}$ & Tracing & 40 / CMY & 75 lpi & $\mathrm{di}: 8^{\circ}$ & & & 10 & 2.08 & (4.27) & 0 & 2.12 & (4.36) & 2.12 & (4.36) & 2.11 & (4.33) \\
\hline $\mathrm{R}$ & Inkjet & Coated & $729 / \mathrm{CMY}$ & 50 1pi & $45^{\circ}: 0^{\circ}$ & 0.85 & $(1.80)$ & 2.4 & 0.87 & (1.73) & 0.1 & 0.91 & (1.69) & 0.9 & (1.69) & 1.00 & (1.92) \\
\hline $\mathrm{S}$ & Inkjet & Coated & 729 / CMY & 75 lpi & $45^{\circ}: 0^{\circ}$ & 0.69 & $(1.50)$ & 5.5 & 0.84 & (1.69) & 0 & 1.16 & (2.15) & 1.16 & (2.15) & 1.37 & (2.48) \\
\hline $\mathrm{T}$ & Inkjet & Coated & 729 / CMY & 100 lpi & $45^{\circ}: 0^{\circ}$ & 0.63 & $(1.22)$ & 6.4 & 0.88 & (1.73) & 0 & 1.32 & (2.31) & 1.32 & (2.31) & 1.52 & $(2.61)$ \\
\hline $\mathrm{U}$ & Inkjet & Coated & $729 / \mathrm{CMY}$ & 125 lpi & $45^{\circ}: 0^{\circ}$ & 0.69 & (1.39) & 10 & 0.92 & (1.70) & 0 & 1.52 & $(2.56)$ & 1.52 & (2.56) & 1.72 & (2.86) \\
\hline $\mathrm{V}$ & Offset & Coated & $729 / \mathrm{CMY}$ & 76 lpi & $45^{\circ}: 0^{\circ}$ & 0.91 & (1.89) & 1.5 & 0.63 & (1.36) & 0.5 & 0.81 & $(1.71)$ & 1.26 & $(2.47)$ & 1.20 & $(2.28)$ \\
\hline $\mathrm{W}$ & Offset & Coated & $729 / \mathrm{CMY}$ & 152 lpi & $45^{\circ}: 0^{\circ}$ & 0.76 & $(1.60)$ & 1.9 & 0.90 & (1.83) & 0.2 & 1.00 & (2.03) & 0.98 & (1.79) & 1.06 & (1.94) \\
\hline $\mathrm{X}$ & Inkjet & MP101 & 625 / CMYK & 100 lpi & $45^{\circ}: 0^{\circ}$ & 0.66 & $(1.62)$ & 14 & 1.13 & $(2.75)$ & 0 & 1.28 & (2.91) & 1.28 & $(2.91)$ & 1.35 & (3.07) \\
\hline $\bar{Y}$ & Inkjet & MP101 & 625 / CMYG & 120 lpi & $45^{\circ}: 0^{\circ}$ & 0.31 & $(0.79)$ & 100 & 0.54 & (1.11) & 0 & 0.67 & (1.30) & 0.67 & (1.30) & 0.69 & (1.40) \\
\hline Z & Inkjet & MP101 & 625 / BOYM & 120 lpi & $45^{\circ}: 0^{\circ}$ & 0.36 & $(0.95)$ & 100 & 0.60 & (1.32) & 0 & 0.85 & (1.85) & 0.85 & (1.85) & 0.96 & (2.03) \\
\hline
\end{tabular}

${ }^{\mathrm{a}}$ Relative ink thickness 1 (maximal ink thickness); ${ }^{\mathrm{b}}$ Relative ink thickness $3 / 4$; ${ }^{\mathrm{c}}$ Relative ink thickness $1 / 2$; ${ }^{\mathrm{d}}$ Relative ink thickness $1 / 4$; 